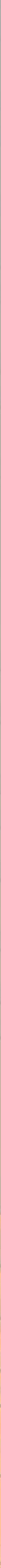




\title{
Parity and symmetry in intersection and ordinary cohomology
}

\author{
Shenghao Sun and Weizhe Zheng
}

To the memory of Torsten Ekedahl

\begin{abstract}
We show that the Galois representations provided by $\ell$-adic cohomology of proper smooth varieties, and more generally by $\ell$-adic intersection cohomology of proper varieties, over any field, are orthogonal or symplectic according to the degree. We deduce this from a preservation result of orthogonal and symplectic pure perverse sheaves by proper direct image. We show, moreover, that the subgroup of the Grothendieck group generated by orthogonal pure perverse sheaves of even weights and symplectic pure perverse sheaves of odd weights are preserved by Grothendieck's six operations. Over a finite field, we deduce parity and symmetry results for Jordan blocks appearing in the Frobenius action on intersection cohomology of proper varieties, and virtual parity results for the Frobenius action on ordinary cohomology of arbitrary varieties.
\end{abstract}

\section{Introduction}

The $n$-th cohomology of a compact Kähler manifold $X$ is equipped with a pure Hodge structure of weight $n$,

$$
\mathrm{H}^{n}(X, \mathbb{Q}) \otimes_{\mathbb{Q}} \mathbb{C}=\bigoplus_{p+q=n} \mathrm{H}^{p q},
$$

where $\mathrm{H}^{p q} \simeq \mathrm{H}^{q}\left(X, \Omega_{X}^{p}\right)$ satisfies $\overline{\mathrm{H}^{p q}}=\mathrm{H}^{q p}$. In particular, $\mathrm{H}^{n}(X, \mathbb{Q})$ is evendimensional for $n$ odd. Hodge decomposition and Hodge symmetry extend to proper smooth schemes over $\mathbb{C}$ [Deligne 1968, Proposition 5.3] by Chow's lemma and resolution of singularities. Thus, in this case, $\mathrm{H}^{n}(X(\mathbb{C}), \mathbb{Q})$ is also even-dimensional

Sun was partially supported by ANR grant G-FIB. Zheng was partially supported by China's Recruitment Program of Global Experts; National Natural Science Foundation of China grant 11321101; National Center for Mathematics and Interdisciplinary Sciences and Hua Loo-Keng Key Laboratory of Mathematics, Chinese Academy of Sciences.

MSC2010: primary 14F20; secondary 14G15, 14F43, 14G25, $11 \mathrm{E} 81$.

Keywords: $\ell$-adic cohomology, intersection cohomology, Galois representation, symmetric form, alternating form, pure perverse sheaf, decomposition theorem, horizontal complex, alteration, Deligne-Mumford stack, Grothendieck-Witt group. 
for $n$ odd. Moreover, the pure Hodge structure of weight $n$ on $\mathrm{H}^{n}(X(\mathbb{C}), \mathbb{Q})$ is polarizable, in the sense that there exists a morphism of Hodge structures

$$
\mathrm{H}^{n}(X(\mathbb{C}), \mathbb{Q}) \otimes \mathrm{H}^{n}(X(\mathbb{C}), \mathbb{Q}) \rightarrow \mathbb{Q}(-n),
$$

symmetric for $n$ even and alternating for $n$ odd, satisfying certain positivity conditions, which implies that the pairing is perfect.

Now let $\bar{k}$ be a separably closed field of characteristic $p \geq 0$ and let $\ell \neq p$ be a prime number. For a projective smooth scheme $X$ of finite type over $\bar{k}$, the hard Lefschetz theorem [Deligne 1980, Théorème 4.1.1] and Poincaré duality equip the $n$-th $\ell$-adic cohomology $\mathrm{H}^{n}\left(X, \mathbb{Q}_{\ell}\right)$ of $X$ with a nondegenerate bilinear form that is symmetric for $n$ even and alternating for $n$ odd. In particular, $\mathrm{H}^{n}\left(X, \mathbb{Q}_{\ell}\right)$ is even-dimensional for $n$ odd. Deligne predicts, in a remark following [1980, Corollaire 4.1.5], that the evenness of the odd-degree Betti numbers should hold more generally for proper smooth schemes over $\bar{k}$. This was recently shown by Suh [2012, Corollary 2.2.3] using crystalline cohomology.

The goal of this article is to study problems of parity and symmetry in more general settings, including symmetry of Galois actions on cohomology. Our approach is different from that of Suh as we do not use $p$-adic cohomology.

For a general scheme $X$ of finite type over $\bar{k}$, the $n$-th cohomology $\mathrm{H}^{n}\left(X, \mathbb{Q}_{\ell}\right)$ is not "pure" and not necessarily even-dimensional for $n$ odd. Before going into results for such mixed situations, let us first state our results in the pure case for intersection cohomology.

Theorem 1.1. Let $k$ be an arbitrary field of characteristic $p \geq 0$ and let $\bar{k}$ be its separable closure. Let $X$ be a proper, equidimensional scheme over $k$. Then, for $n$ even (resp. odd), the $n$-th $\ell$-adic intersection cohomology group admits a $\operatorname{Gal}(\bar{k} / k)$-equivariant symmetric (resp. alternating) perfect pairing

$$
\operatorname{IH}^{n}\left(X_{\bar{k}}, \mathbb{Q}_{\ell}\right) \otimes \operatorname{IH}^{n}\left(X_{\bar{k}}, \mathbb{Q}_{\ell}\right) \rightarrow \mathbb{Q}_{\ell}(-n) .
$$

Here $\operatorname{Gal}(\bar{k} / k)$ denotes the Galois group of $k$, and $X_{\bar{k}}=X \otimes_{k} \bar{k}$.

By definition, we have $\operatorname{IH}^{n}\left(X_{\bar{k}}, \mathbb{Q}_{\ell}\right)=\mathrm{H}^{n-d}\left(X_{\bar{k}}, \mathrm{IC}_{X}\right)$, where $\mathrm{IC}_{X}=j_{! *}\left(\mathbb{Q}_{\ell}[d]\right)$ and $d=\operatorname{dim}(X)$, and where $j: U \rightarrow X$ is an open dense immersion such that $U_{\text {red }}$ is regular. For $X$ proper smooth, we have $\operatorname{IH}^{n}\left(X_{\bar{k}}, \mathbb{Q}_{\ell}\right)=\mathrm{H}^{n}\left(X_{\bar{k}}, \mathbb{Q}_{\ell}\right)$, and the theorem takes the following form. The statement was suggested to us by Takeshi Saito. One may compare such pairings with polarizations of pure Hodge structures, mentioned at the beginning of the Introduction.

Corollary 1.2. Let $X$ be a proper smooth scheme over $k$. Then, for $n$ even (resp. odd), the $n$-th $\ell$-adic cohomology group admits a $\mathrm{Gal}(\bar{k} / k)$-equivariant symmetric (resp. alternating) perfect pairing

$$
\mathrm{H}^{n}\left(X_{\bar{k}}, \mathbb{Q}_{\ell}\right) \otimes \mathrm{H}^{n}\left(X_{\bar{k}}, \mathbb{Q}_{\ell}\right) \rightarrow \mathbb{Q}_{\ell}(-n)
$$


Ignoring Galois actions, we obtain the following corollary. For $X$ proper smooth, this gives another proof of Suh's result mentioned earlier.

Corollary 1.3. Let $X$ be a proper, equidimensional scheme over $k$. Then $\operatorname{IH}^{n}\left(X_{\bar{k}}, \mathbb{Q}_{\ell}\right)$ is even-dimensional for $n$ odd.

To demonstrate the strength of Theorem 1.1, we give a reformulation in the case where $k=\mathbb{F}_{q}$ is a finite field. In this case, the Galois action is determined by Frobenius action. We let $\operatorname{Frob}_{q} \in \operatorname{Gal}\left(\overline{\mathbb{F}}_{q} / \mathbb{F}_{q}\right)$ denote the geometric Frobenius $x \mapsto x^{1 / q}$. The eigenvalues of $\operatorname{Frob}_{q}$ acting on $\operatorname{IH}^{n}\left(X_{\overline{\mathbb{F}}_{q}}, \mathbb{Q}_{\ell}\right)$ are $q$-Weil integers of weight $n$, by which we mean algebraic integers $\lambda$ such that, for every embedding $\alpha: \mathbb{Q}(\lambda) \rightarrow \mathbb{C}$, we have $|\alpha(\lambda)|^{2}=q^{n}$. We let $\mu_{\lambda} \in \mathbb{Z}_{\geq 0}$ denote the multiplicity of the eigenvalue $\lambda$ for the action of $\operatorname{Frob}_{q}$ on $\operatorname{IH}^{n}\left(X_{\overline{\mathbb{F}}_{q}}, \mathbb{Q}_{\ell}\right)$. In other words, we set

$$
\operatorname{det}\left(1-T \operatorname{Frob}_{q} \mid \mathrm{IH}^{n}\left(X_{\overline{\mathbb{F}}_{q}}, \mathbb{Q}_{\ell}\right)\right)=\prod_{\lambda}(1-\lambda T)^{\mu_{\lambda}} .
$$

For $e \geq 1$, let $\mu_{\lambda, e} \in \mathbb{Z}_{\geq 0}$ denote the number of $e \times e$ Jordan blocks with eigenvalue $\lambda$ in the Jordan normal form of $\operatorname{Frob}_{q}$ acting on $\operatorname{IH}^{n}\left(X_{\overline{\mathbb{F}}_{q}}, \overline{\mathbb{Q}}_{\ell}\right)$. Then $\mu_{\lambda}=\sum_{e \geq 1} e \mu_{\lambda, e}$.

Corollary 1.4. Let $X$ be a proper, equidimensional scheme over $\mathbb{F}_{q}$. In the above notation, $\mu_{\lambda, e}=\mu_{q^{n} / \lambda, e}$. Moreover, $\mu_{\sqrt{q^{n}}, e}$ and $\mu_{-\sqrt{q^{n}}, e}$ are even for $n+e$ even. In particular, $\mu_{\lambda}=\mu_{q^{n} / \lambda}$ and, for $n$ odd, $\mu_{\sqrt{q^{n}}}$ and $\mu_{-\sqrt{q^{n}}}$ are even.

The last statement of Corollary 1.4 implies that, for $n$ odd, $\operatorname{det}\left(\operatorname{Frob}_{q} \mid \mathrm{IH}^{n}\left(X_{\overline{\mathbb{F}}_{q}}, \mathbb{Q}_{\ell}\right)\right)=q^{n b_{n} / 2}$, $\operatorname{det}\left(1-T \operatorname{Frob}_{q} \mid \operatorname{IH}^{n}\left(X_{\overline{\mathbb{F}}_{q}}, \mathbb{Q}_{\ell}\right)\right)=q^{n b_{n} / 2} T^{b_{n}} \operatorname{det}\left(1-q^{-n} T^{-1} \operatorname{Frob}_{q} \mid \operatorname{IH}^{n}\left(X_{\overline{\mathbb{F}}_{q}}, \mathbb{Q}_{\ell}\right)\right)$, where $b_{n}=\operatorname{dim} \operatorname{IH}^{n}\left(X_{\overline{\mathbb{F}}_{q}}, \mathbb{Q}_{\ell}\right)$. It also implies that $\operatorname{dim} \operatorname{IH}^{n}\left(X_{\overline{\mathbb{F}}_{q}}, \mathbb{Q}_{\ell}\right)=\sum_{\lambda} \mu_{\lambda}$ is even. Remark 1.5. Some special cases of the last statement of Corollary 1.4 were previously known.

(1) Gabber's theorem on the independence of $\ell$ for intersection cohomology [Fujiwara 2002, Theorem 1] states that (1-3-1) belongs to $\mathbb{Z}[T]$ and is independent of $\ell$. The fact that (1-3-1) belongs to $\mathbb{Q}[T]$ implies $\mu_{\lambda}=\mu_{\lambda^{\prime}}$, for $\lambda$ and $\lambda^{\prime}$ in the same $\operatorname{Gal}(\overline{\mathbb{Q}} / \mathbb{Q})$-orbit. In particular, $\mu_{\lambda}=\mu_{q^{n} / \lambda}$, and, if $q$ is not a square and $n$ is odd, $\mu_{\sqrt{q^{n}}}=\mu_{-\sqrt{q^{n}}}$, so that $\operatorname{dim} \operatorname{IH}^{n}\left(X_{\overline{\mathbb{F}}_{q}}, \mathbb{Q}_{\ell}\right)=\sum_{\lambda} \mu_{\lambda}$ is even in this case.

(2) For $X$ proper smooth, the fact that $\mu_{\sqrt{q^{n}}}$ and $\mu_{-\sqrt{q^{n}}}$ are even for $n$ odd follows from a theorem of Suh [2012, Theorem 3.3.1].

Remark 1.6. The first two statements of Corollary 1.4 are consistent with the conjectural semisimplicity of the Frobenius action on $\mathrm{IH}^{n}\left(X_{\overline{\mathbb{F}}_{q}}, \mathbb{Q}_{\ell}\right)$ (namely, $\mu_{\lambda, e}=0$ for $e \geq 2$ ), which would follow from the standard conjectures. To see this implication, let $X^{\prime} \rightarrow X$ be a surjective generically finite morphism such that $X^{\prime}$ is projective 
smooth over $\mathbb{F}_{q}$, which exists by de Jong's alterations [1996, Theorem 4.1]. Then $\operatorname{IH}^{n}\left(X_{\overline{\mathbb{F}}_{q}}, \mathbb{Q}_{\ell}\right)$ as a $\operatorname{Gal}\left(\overline{\mathbb{F}}_{q} / \mathbb{F}_{q}\right)$-module is a direct summand of $\mathrm{H}^{n}\left(\left(X^{\prime}\right)_{\overline{\mathbb{F}}_{q}}, \mathbb{Q}_{\ell}\right){ }^{1}$ The semisimplicity of the Frobenius action on $\mathrm{H}^{n}\left(\left(X^{\prime}\right)_{\overline{\mathbb{F}}_{q}}\right.$, $\left.\mathbb{Q}_{\ell}\right)$ would follow from the Lefschetz type standard conjecture for $X^{\prime}$ and the Hodge type standard conjecture for $X^{\prime} \times X^{\prime}$ [Kleiman 1994, Theorem 5.6(2)].

To prove Theorem 1.1, we may assume that $k$ is finitely generated over its prime field. We will keep this assumption in the rest of the Introduction. This includes notably the case of a number field. We deduce Theorem 1.1 from a relative result with coefficients. In the case where $k$ is a finite field, the coefficients are pure perverse sheaves. In the general case, we apply the formalism of pure horizontal perverse sheaves of Annette Huber [1997], as extended by Sophie Morel [2012]. Since the proofs are the same in the two cases, we recommend readers not familiar with horizontal perverse sheaves to concentrate on the case of a finite field and to ignore the word "horizontal". Unless otherwise stated, we will only consider the middle perversity. We let $\overline{\mathbb{Q}}_{\ell}$ denote the algebraic closure of $\mathbb{Q}_{\ell}$.

Definition 1.7. Let $X$ be a scheme of finite type over $k$ and let $A \in \mathrm{D}_{c}^{b}\left(X, \overline{\mathbb{Q}}_{\ell}\right)$ be a horizontal perverse sheaf on $X$, pure of weight $w$. We say that $A$ is orthogonal if there exists a symmetric perfect pairing $A \otimes A \rightarrow K_{X}(-w)$. We say that $A$ is symplectic if there exists an alternating perfect pairing $A \otimes A \rightarrow K_{X}(-w)$.

Here $K_{X}=\mathrm{R} a_{X}^{!} \overline{\mathbb{Q}}_{\ell}$ is the dualizing complex on $X$, where $a_{X}: X \rightarrow \operatorname{Spec}(k)$ is the structural morphism.

Theorem 1.8 (special case of Theorem 5.1.5). Let $f: X \rightarrow Y$ be a proper morphism of schemes of finite type over $k$ and let $A \in \mathrm{D}_{c}^{b}\left(X, \overline{\mathbb{Q}}_{\ell}\right)$ be an orthogonal (resp. symplectic) pure horizontal perverse sheaf on $X$. Then

$$
\mathrm{R} f_{*} A \simeq \bigoplus_{n}\left({ }^{p} \mathrm{R}^{n} f_{*} A\right)[-n],
$$

and ${ }^{p} \mathrm{R}^{n} f_{*} A$ is orthogonal (resp. symplectic) for $n$ even and symplectic (resp. orthogonal) for $n$ odd.

Recall that the Beilinson-Bernstein-Deligne-Gabber decomposition theorem [Beilinson et al. 1982, Théorème 5.4.5] implies that (1-8-1) holds after base change to the algebraic closure $\bar{k}$ of $k$.

Theorem 1.1 follows from Theorem 1.8 applied to the morphism $a_{X}: X \rightarrow \operatorname{Spec}(k)$. Even if one is only interested in Theorem 1.1, our proof leads one to consider the relative situation of Theorem 1.8.

Next we state results for operations that do not necessarily preserve pure (horizontal) complexes. For a scheme $X$ of finite type over $k$, we let $\mathrm{K}_{\text {orth }}\left(X, \overline{\mathbb{Q}}_{\ell}\right)$

\footnotetext{
${ }^{1}$ This argument is also used in Gabber's proof of the integrality of (1-3-1).
} 
denote the subgroup of the Grothendieck group $\mathrm{K}\left(X, \overline{\mathbb{Q}}_{\ell}\right)$ of $\mathrm{D}_{c}^{b}\left(X, \overline{\mathbb{Q}}_{\ell}\right)$ generated by orthogonal pure horizontal perverse sheaves of even weights and symplectic pure horizontal perverse sheaves of odd weights.

Theorem 1.9 (special case of Theorem 5.2.2). Grothendieck's six operations preserve $\mathrm{K}_{\mathrm{orth}}$.

Note that the preservation of $\mathrm{K}_{\text {orth }}$ by each of the six operations is nontrivial. The crucial case turns out to be the preservation by the "extension by zero" functor $j$ ! for certain open immersions $j$ (Proposition 4.3.1).

As Michel Gros points out, one may compare these theorems to Morihiko Saito's theory [1990] of mixed Hodge modules. By definition, a mixed Hodge module admits a weight filtration for which the graded pieces are polarizable pure Hodge modules. One may compare Definition 1.7 to polarizable pure Hodge modules.

Let us state a consequence of Theorem 1.9 on Galois action on cohomology in the case where $k=\mathbb{F}_{q}$ is a finite field. Let $X$ be a scheme of finite type over $\mathbb{F}_{q}$. The eigenvalues of $\operatorname{Frob}_{q}$ acting on $\mathrm{H}^{*}\left(X_{\overline{\mathbb{F}}_{q}}, \mathbb{Q}_{\ell}\right)$ are $q$-Weil integers ${ }^{2}$ of integral weights. We let $m_{\lambda} \in \mathbb{Z}$ denote the multiplicity of the eigenvalue $\lambda$. In other words, we set

$$
\prod_{n} \operatorname{det}\left(1-T \operatorname{Frob}_{q} \mid \mathrm{H}^{n}\left(X_{\overline{\mathbb{F}}_{q}}, \mathbb{Q}_{\ell}\right)\right)^{(-1)^{n}}=\prod_{\lambda}(1-\lambda T)^{m_{\lambda}} \text {. }
$$

Applying the theorem to $\left(a_{X}\right)_{*}$, where $a_{X}: X \rightarrow \operatorname{Spec}\left(\mathbb{F}_{q}\right)$, we obtain the following.

Corollary 1.10. Let $X$ be a scheme of finite type over $\mathbb{F}_{q}$. In the above notation, $m_{\lambda}=m_{q^{w} / \lambda}$ for every $q$-Weil integer $\lambda$ of weight $w$, and, for $w$ odd, $m_{\sqrt{q^{w}}}$ and $m_{-\sqrt{q^{w}}}$ are even. In particular, for $w$ odd, $\sum_{\lambda} m_{\lambda}$ (where $\lambda$ runs through $q$-Weil integers of weight $w)$, the dimension of the weight-w part of $\mathrm{H}^{*}\left(X_{\overline{\mathbb{F}}_{q}}, \mathbb{Q}_{\ell}\right)$, is even.

Theorem 1.9 also implies analogues of Corollary 1.10 for compactly supported cohomology $\mathrm{H}_{c}^{*}\left(X, \mathbb{Q}_{\ell}\right)$, and, if $X$ is equidimensional, intersection cohomology $\mathrm{IH}^{*}\left(X, \mathbb{Q}_{\ell}\right)$ and compactly supported intersection cohomology $\mathrm{IH}_{c}^{*}\left(X, \mathbb{Q}_{\ell}\right)$. In the case of $\mathrm{H}_{c}^{*}\left(X, \mathbb{Q}_{\ell}\right)$, the analogue of (1-9-1) is the inverse of the zeta function, and the analogue of Corollary 1.10 was established by Suh [2012, Theorem 3.3.1] using rigid cohomology.

Remark 1.11. Some special cases of Corollary 1.10 were previously known. By Gabber's theorem on the independence of $\ell$ [Fujiwara 2002, Theorem 2], (1-9-1) belongs to $\mathbb{Q}(T)$ and is independent of $\ell$. The fact that (1-9-1) belongs to $\mathbb{Q}(T)$ implies that $m_{\lambda}=m_{\lambda^{\prime}}$, for $\lambda$ and $\lambda^{\prime}$ in the same $\operatorname{Gal}(\overline{\mathbb{Q}} / \mathbb{Q})$-orbit. In particular, $m_{\lambda}=m_{q^{w} / \lambda}$ for every $q$-Weil integer $\lambda$ of weight $w$, and, if $q$ is not a square and $w$ is odd, $m_{\sqrt{q^{w}}}=m_{-\sqrt{q^{w}}}$ so that $\sum_{\lambda} m_{\lambda}$ (where $\lambda$ runs through $q$-Weil integers of weight $w$ ) is even in this case.

\footnotetext{
${ }^{2}$ The integrality is a special case of [Zheng 2008, Variante 5.1].
} 
One ingredient in the proof of Theorem 1.9 is de Jong's alterations. Note that, even for a finite étale cover $f: X \rightarrow Y$, one cannot recover the parity of an object on $Y$ from the parity of its pullback to $X$. More precisely, for an element $A$ in the Grothendieck group of mixed horizontal perverse sheaves on $Y$ such that $f^{*} A \in \mathrm{K}_{\text {orth }}$, we do not have $A \in \mathrm{K}_{\text {orth }}$ in general. We use equivariant alterations to compensate for this loss of information. In order to better deal with the equivariant situation, we will work systematically with Deligne-Mumford stacks in the main text. We note however that the proofs of Theorems 1.1 and 1.8 (and the corollaries to Theorem 1.1) do not depend on stacks, and readers only interested in these results may, in the corresponding portions of the text (Sections 2, 3 and 5.1), assume every stack to be a scheme.

The paper is organized as follows. In Section 2, we study symmetry of complexes and perverse sheaves over a general field. In Section 3, we study symmetry and decomposition of pure complexes over a finite field and prove Theorem 1.8 in this case. In Section 4, we study symmetry in Grothendieck groups over a finite field and prove Theorem 1.9 in this case. In Section 5, we study symmetry of horizontal complexes over a general field finitely generated over its prime field and finish the proof of the theorems. In the Appendix, we collect some general symmetry properties in categories with additional structures, which are used in the main body of the paper.

Conventions. Unless otherwise indicated, $X, Y$, etc., will denote Deligne-Mumford stacks of finite presentation (i.e., of finite type and quasiseparated) over a base field $k$; this rules out stacks such as $B \mathbb{Z}$. We recall that, for schemes, being of finite presentation over $k$ is the same as being of finite type over $k$.

We let $\ell$ denote a prime number invertible in $k$, and we let $\mathrm{D}_{c}^{b}\left(X, \overline{\mathbb{Q}}_{\ell}\right)$ denote the derived category of $\overline{\mathbb{Q}}_{\ell}$-complexes on $X$. We refer the reader to [Zheng 2015b] for the construction of $\mathrm{D}_{c}^{b}\left(X, \overline{\mathbb{Q}}_{\ell}\right)$ and of Grothendieck's six operations. We denote by $a_{X}: X \rightarrow \operatorname{Spec}(k)$ the structural morphism, by $K_{X}:=\mathrm{R} a_{X}^{!} \overline{\mathbb{Q}}_{\ell}$ the dualizing complex on $X$, and by $D_{X}$ the dualizing functor $D_{K_{X}}:=\mathrm{R} \mathscr{H} O \operatorname{Oom}\left(-, K_{X}\right)$.

As mentioned above, we will only consider the middle perversity unless otherwise stated. We let $\operatorname{Perv}\left(X, \overline{\mathbb{Q}}_{\ell}\right) \subseteq \mathrm{D}_{c}^{b}\left(X, \overline{\mathbb{Q}}_{\ell}\right)$ denote the full subcategory of perverse $\overline{\mathbb{Q}}_{\ell}$-sheaves on $X$. For a separated quasifinite morphism $f: X \rightarrow Y$, the middle extension functor $f_{!_{*}}: \operatorname{Perv}\left(X, \overline{\mathbb{Q}}_{\ell}\right) \rightarrow \operatorname{Perv}\left(Y, \overline{\mathbb{Q}}_{\ell}\right)$ is the image of the supportforgetting morphism ${ }^{p} \mathscr{H}^{0} f_{!} \rightarrow{ }^{p} \mathscr{H}^{0} \mathrm{R} f_{*}$.

Throughout the article, we let $\sigma$ and $\sigma^{\prime}$ represent elements of $\{ \pm 1\}$.

\section{Symmetry of complexes and perverse sheaves}

In this section, we study symmetry properties of $\overline{\mathbb{Q}}_{\ell}$-complexes, namely objects of $\mathrm{D}_{c}^{b}\left(X, \overline{\mathbb{Q}}_{\ell}\right)$, over an arbitrary field $k$. In Section 2.1, we define $\sigma$-self-dual complexes and we study their behavior under operations that commute with duality. In 
Section 2.2, we analyze $\sigma$-self-dual semisimple perverse sheaves. In this generality none of the results is difficult, but they will be used quite often in the sequel.

2.1. Symmetry of complexes. The tensor product endows $\mathrm{D}_{c}^{b}\left(X, \overline{\mathbb{Q}}_{\ell}\right)$ with the structure of a closed symmetric monoidal structure. The definition below only makes use of the symmetry constraint $c_{A B}: A \otimes B \stackrel{\sim}{\rightarrow} B \otimes A$ and the internal mapping object R $\mathscr{H}$ om.

Definition 2.1.1 ( $\sigma$-self-dual complexes). Let $A, C \in \mathrm{D}_{c}^{b}\left(X, \overline{\mathbb{Q}}_{\ell}\right)$. We say that $A$ is 1-self-dual with respect to $C$ (resp. -1-self-dual with respect to $C$ ) if there exists a pairing $A \otimes A \rightarrow C$ that is

- symmetric (resp. alternating), in the sense that the diagram

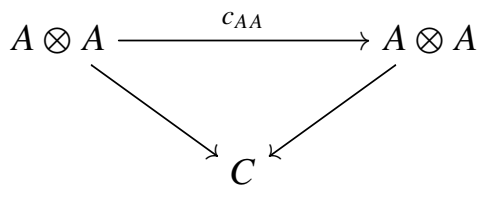

commutes (resp. anticommutes), and

- perfect, in the sense that the pairing induces an isomorphism $A \stackrel{\sim}{\longrightarrow} D_{C} A:=$ $\mathrm{R} \mathscr{H} O m(A, C)$.

We say that $A$ is self-dual with respect to $C$ if there exists an isomorphism $A \stackrel{\sim}{\rightarrow} D_{C} A$.

The symmetry of the pairing $A \otimes A \rightarrow C$ can also be expressed in terms of the induced morphism $f: A \rightarrow D_{C} A$. In fact, the diagram (2-1-1) $\sigma$-commutes if and only if the diagram

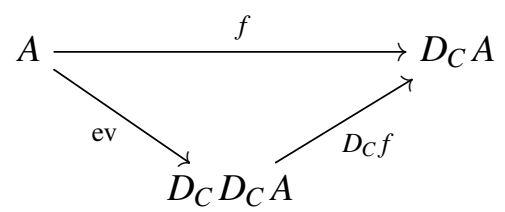

$\sigma$-commutes (Lemma A.4.2).

Remark 2.1.2. Similarly one can define self-dual and $\sigma$-self-dual $E_{\lambda}$-complexes, where $E_{\lambda}$ is any algebraic extension of $\mathbb{Q}_{\ell}$. Note that, for $A, C \in \mathrm{D}_{c}^{b}\left(X, E_{\lambda}\right)$, $A$ is self-dual (resp. $\sigma$-self-dual) with respect to $C$ if and only if $A \otimes_{E_{\lambda}} \overline{\mathbb{Q}}_{\ell}$ satisfies the same property with respect to $C \otimes_{E_{\lambda}} \overline{\mathbb{Q}}_{\ell}$. Indeed, the "only if" part is obvious. To see the "if" part, consider $U=\operatorname{Isom}\left(A_{\bar{k}}, D_{C} A_{\bar{k}}\right)$ as in Lemma 2.1.3 below. Here $A_{\bar{k}}$ denotes the pullback of $A$ to $X_{\bar{k}}$. Recall that rational points form a Zariski dense subset of any affine space over an infinite field. If $A \otimes_{E_{\lambda}} \overline{\mathbb{Q}}_{\ell}$ is self-dual (resp. $\sigma$-self-dual) with respect to $C \otimes_{E_{\lambda}} \overline{\mathbb{Q}}_{\ell}$, then $U \cap V$ is nonempty, and hence has an $E_{\lambda}$-point. Here $V \subseteq \operatorname{Hom}\left(A_{\bar{k}}, D_{C} A_{\bar{k}}\right)$ is represented by the $E_{\lambda}$-vector subspace, 
image of morphisms (resp. $\sigma$-symmetric morphisms) $A \rightarrow D_{C} A$. For the above reason, we will work almost exclusively with $\overline{\mathbb{Q}}_{\ell}$-complexes.

Lemma 2.1.3. Let $A, B \in \mathrm{D}_{c}^{b}\left(X, E_{\lambda}\right)$ satisfy $\operatorname{dim}_{E_{\lambda}} \operatorname{Hom}(A, B)<\infty$. Then there exists a Zariski open subscheme $U=\operatorname{Isom}(A, B)$ of the affine space $\operatorname{Hom}(A, B)$ over $E_{\lambda}$ represented by the $E_{\lambda}$-vector space $\operatorname{Hom}(A, B)$ such that, for any algebraic extension $E_{\lambda}^{\prime}$ of $E_{\lambda}$, the subset $U\left(E_{\lambda}^{\prime}\right)$ is the set of isomorphisms $A \otimes_{E_{\lambda}} E_{\lambda}^{\prime} \stackrel{\sim}{\longrightarrow}$ $B \otimes_{E_{\lambda}} E_{\lambda}^{\prime}$.

Proof. Assume $A, B \in \mathrm{D}^{[a, b]}$. Choose a stratification of $X$ by connected, geometrically unibranch substacks such that the restrictions of $\mathscr{H}^{n} A$ and $\mathscr{H}^{n} B$ to each stratum are lisse sheaves. Choose a geometric point $x$ in each stratum. Then a morphism $f: A \otimes_{E_{\lambda}} E_{\lambda}^{\prime} \rightarrow B \otimes_{E_{\lambda}} E_{\lambda}^{\prime}$ is an isomorphism if and only if $\mathscr{H}^{n} f_{x}$ is for every $n \in[a, b]$ and every $x$ in the finite collection. Then $\operatorname{Isom}(A, B)$ is the intersection of the pullbacks of the open subsets $\operatorname{Isom}\left(\mathscr{H}^{n} A_{x}, \mathscr{H}^{n} B_{x}\right) \subseteq \operatorname{Hom}\left(\mathscr{H}^{n} A_{x}, \mathscr{H}^{n} B_{x}\right)$.

We will mostly be interested only in duality with respect to Tate twists $K_{X}(-w)$, $w \in \mathbb{Z}$, of the dualizing complex $K_{X}=\mathrm{R} a_{X} ! \overline{\mathbb{Q}}_{\ell}$. In this case, the evaluation morphism $A \rightarrow D_{K_{X}(-w)} D_{K_{X}(-w)} A$ is an isomorphism. The functor $D_{K_{X}(-w)}$ preserves perverse sheaves. We will sometimes write $K$ for $K_{X}$ when no confusion arises.

In the rest of this subsection, we study the behavior of $\sigma$-self-dual complexes under operations that commute with the dualizing functors (up to shift and twist). The results are mostly formal, but for completeness we provide a proof for each result, based on general facts on symmetry in categories collected in the Appendix. Readers willing to accept these results may skip the proofs.

Most of the proofs consist of showing that the natural isomorphism representing the commutation of the functor in question with duality is symmetric in the sense of Definition A.3.3. It then follows from Lemma A.3.9 that the functor in question preserves $\sigma$-self-dual objects.

Remark 2.1.4 (preservation of $\sigma$-self-dual complexes). Let $f: X \rightarrow Y$ be a morphism. Let $w, w^{\prime} \in \mathbb{Z}$.

(1) For $n \in \mathbb{Z}$, Tate twist $A \mapsto A(n)$ carries $\overline{\mathbb{Q}}_{\ell}$-complexes $\sigma$-self-dual with respect to $C$ to $\overline{\mathbb{Q}}_{\ell}$-complexes $\sigma$-self-dual with respect to $C(2 n)$; the shift functor $A \mapsto A[n]$ carries $\overline{\mathbb{Q}}_{\ell}$-complexes $\sigma$-self-dual with respect to $C$ to $\overline{\mathbb{Q}}_{\ell}$-complexes $(-1)^{n} \sigma$-selfdual with respect to $C[2 n]$.

This follows from Lemma A.5.6.

(2) $D_{X}$ carries $\overline{\mathbb{Q}}_{\ell}$-complexes $\sigma$-self-dual with respect to $K_{X}(-w)$ to $\overline{\mathbb{Q}}_{\ell}$-complexes $\sigma$-self-dual with respect to $K_{X}(w)$.

Since $D_{X} A \simeq\left(D_{K_{X}(-w)} A\right)(w)$, the assertion follows from (1).

(3) Assume that $f$ is proper. Then $\mathrm{R} f_{*}: \mathrm{D}_{c}^{b}\left(X, \overline{\mathbb{Q}}_{\ell}\right) \rightarrow \mathrm{D}_{c}^{b}\left(Y, \overline{\mathbb{Q}}_{\ell}\right)$ preserves $\sigma$-selfdual objects with respect to $K(-w)$. In other words, $\mathrm{R} f_{*}$ carries $\overline{\mathbb{Q}}_{\ell}$-complexes 
$\sigma$-self-dual with respect to $K_{X}(-w)$ to $\overline{\mathbb{Q}}_{\ell}$-complexes $\sigma$-self-dual with respect to $K_{Y}(-w)$.

Since $\mathrm{R} f_{*}$ is a right-lax symmetric functor (Definition A.1.5), the morphism $\mathrm{R} f_{*} D_{X} \rightarrow D_{\mathrm{R} f_{*} K_{X}} \mathrm{R} f_{*}$ is symmetric by Construction A.4.6. Composing with the adjunction map $\mathrm{R} f_{*} K_{X} \simeq \mathrm{R} f_{!} K_{X} \rightarrow K_{Y}$, we obtain a symmetric isomorphism $\mathrm{R} f_{*} D_{X} \stackrel{\sim}{\longrightarrow} D_{Y} \mathrm{R} f_{*}$.

(4) Assume that $f$ is a closed immersion, and let $A \in \mathrm{D}_{c}^{b}\left(X, \overline{\mathbb{Q}}_{\ell}\right)$. Then $A$ is $\sigma$-self-dual with respect to $K_{X}(-w)$ if and only if $f_{*} A$ is $\sigma$-self-dual with respect to $K_{Y}(-w)$.

Since the functor $f_{*}$ is fully faithful in this case, the assertion follows from the proof of (3) above and Lemma A.3.9.

(5) Assume that $f$ is an open immersion and let $A \in \operatorname{Perv}\left(X, \overline{\mathbb{Q}}_{\ell}\right)$ be a perverse sheaf. Then $A$ is $\sigma$-self-dual with respect to $K_{X}(-w)$ if and only if $f_{! *} A$ is $\sigma$-self-dual with respect to $K_{Y}(-w)$.

Since $f_{!}$is a symmetric functor, the morphism $f_{!} D_{X} \rightarrow D_{f_{!} K_{X}} f_{!}$is symmetric by Construction A.4.6. It follows that the composite map in the commutative square

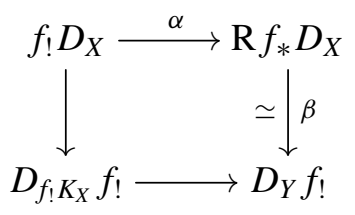

is symmetric. Here the lower horizontal map is given by the adjunction map $f_{!} K_{X} \rightarrow K_{Y}$. Moreover, we have a commutative square

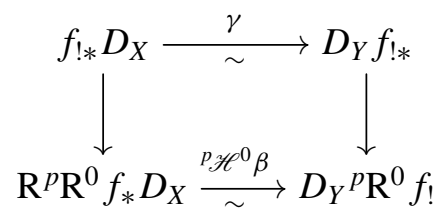

By Lemma A.3.10, $\gamma$ is symmetric. Since the functor $f_{\text {!* }}$ is fully faithful by Lemma 2.1.5 below, it suffices to apply Lemma A.3.9. The "if" part also follows from (8) below.

(6) Let $A \in \mathrm{D}_{c}^{b}\left(X, \overline{\mathbb{Q}}_{\ell}\right)$ be $\sigma$-self-dual with respect to $K_{X}(-w)$ and let $B \in \mathrm{D}_{c}^{b}\left(X^{\prime}, \overline{\mathbb{Q}}_{\ell}\right)$ be $\sigma^{\prime}$-self-dual with respect to $K_{X^{\prime}}\left(-w^{\prime}\right)$. Then the exterior tensor product $A \otimes B$ in $\mathrm{D}_{c}^{b}\left(X \times X^{\prime}, \overline{\mathbb{Q}}_{\ell}\right)$ is $\sigma \sigma^{\prime}$-self-dual with respect to $K_{X \times X^{\prime}}\left(-w-w^{\prime}\right)$.

Since $-\otimes-$ is a symmetric monoidal functor, the Künneth isomorphism (see [Grothendieck 1977, Equation (1.7.6), Proposition 2.3])

$$
D_{X}(-) \otimes D_{X^{\prime}}(-) \stackrel{\sim}{\longrightarrow} D_{K_{X} \otimes K_{X^{\prime}}}(-\otimes-) \simeq D_{X \times X^{\prime}}(-\otimes-)
$$


is symmetric by Construction A.4.6.

(7) If $f$ is smooth, purely of dimension $d$, then $f^{*}[d]$ carries $\overline{\mathbb{Q}}_{\ell}$-complexes $\sigma$-selfdual with respect to $K_{Y}(-w)$ to $\overline{\mathbb{Q}}_{\ell}$-complexes $(-1)^{d} \sigma$-self-dual with respect to $K_{X}(-d-w)$.

Since $f^{*}$ is a symmetric monoidal functor, the isomorphism

$$
f^{*} T^{d} D_{Y} \stackrel{\sim}{\longrightarrow} D_{f^{*} K_{Y}[2 d]} f^{*} T^{d} \simeq D_{K_{X}(-d)} f^{*} T^{d}
$$

is $(-1)^{d}$-symmetric by Construction A.4.6 and Remark A.5.10.

(8) If $X$ and $Y$ are regular, purely of dimension $d$ and $e$, respectively, then $f^{*}[d-e]$ : $\mathrm{D}_{\text {lisse }}^{b}\left(Y, \overline{\mathbb{Q}}_{\ell}\right) \rightarrow \mathrm{D}_{\text {lisse }}^{b}\left(X, \overline{\mathbb{Q}}_{\ell}\right)$ carries $\overline{\mathbb{Q}}_{\ell}$-complexes $\sigma$-self-dual with respect to $K_{Y}(-w)$ to $\overline{\mathbb{Q}}_{\ell}$-complexes $(-1)^{d-e} \sigma$-self-dual with respect to $K_{X}(-(d-e)-w)$. Here $\mathrm{D}_{\text {lisse }}^{b}$ denotes the full subcategory of $\mathrm{D}_{c}^{b}$ consisting of complexes with lisse cohomology sheaves.

Let $r=d-e$. As in (7), the natural transformation

$$
f^{*} T^{r} D_{Y} \rightarrow D_{f^{*} K_{Y}[2 r]} f^{*} T^{r} \simeq D_{K_{X}(-r)} f^{*} T^{r}
$$

is $(-1)^{r}$-symmetric. For $A \in \mathrm{D}_{c}^{b}\left(Y, \overline{\mathbb{Q}}_{\ell}\right)$, this natural transformation can be computed as

$$
f^{*} T^{r} D_{Y} A \simeq f^{*} T^{r} D_{K_{Y}(-r)} A \otimes \mathrm{R} f^{!} \overline{\mathbb{Q}}_{\ell} \stackrel{\alpha}{\longrightarrow} \mathrm{R} f^{!} D_{K_{Y}(-r)} T^{r} A \simeq D_{K_{X}(-r)} f^{*} T^{r} A .
$$

For $A \in \mathrm{D}_{\text {lisse }}^{b}, \alpha$ is an isomorphism.

Similar results hold for self-dual $\overline{\mathbb{Q}}_{\ell}$-complexes.

Lemma 2.1.5. Let $j: U \rightarrow X$ be an immersion. Then the functor $j_{! *}: \operatorname{Perv}\left(U, \overline{\mathbb{Q}}_{\ell}\right) \rightarrow$ $\operatorname{Perv}\left(X, \overline{\mathbb{Q}}_{\ell}\right)$ is fully faithful.

Proof. Let $A$ and $B$ be perverse $\overline{\mathbb{Q}}_{\ell}$-sheaves on $U$, and let $\alpha: \operatorname{Hom}(A, B) \rightarrow$ $\operatorname{Hom}\left(j_{!_{*}} A, j_{! *} B\right)$ be the map induced by $j_{\text {!* }}$. As the composite map

$\operatorname{Hom}(A, B) \stackrel{\alpha}{\longrightarrow} \operatorname{Hom}\left(j_{! *} A, j_{! *} B\right) \stackrel{\beta}{\longrightarrow} \operatorname{Hom}\left({ }^{p} \mathscr{H}^{0} j_{!} A,{ }^{p} \mathscr{H}^{0} R j_{*} B\right) \simeq \operatorname{Hom}\left(j_{!} A, R j_{*} B\right)$

is an isomorphism and $\beta$ is an injection, $\alpha$ is an isomorphism.

Example 2.1.6. Assume that $X$ is equidimensional. We define the intersection complex of $X$ by $\operatorname{IC}_{X}=j_{! *}\left(\mathbb{Q}_{\ell}[d]\right)$, where $j: U \rightarrow X$ is a dominant open immersion such that $U_{\text {red }}$ is regular and $d$ equals $\operatorname{dim} X$. Then by parts (5) and (7) of Remark 2.1.4, $\mathrm{IC}_{X}$ is $(-1)^{d}$-self-dual with respect to $K_{X}(-d)$.

Although we do not need it, let us mention the following stability of $\sigma$-self-dual complexes under nearby cycles.

Remark 2.1.7. Let $S$ be the spectrum of a Henselian discrete valuation ring, of generic point $\eta$ and closed point $s$, on which $\ell$ is invertible. Let $\mathfrak{X}$ be a DeligneMumford stack of finite presentation over $S$. Then the nearby cycle functor 
$\mathrm{R} \Psi: \mathrm{D}_{c}^{b}\left(\mathfrak{X}_{\eta}, \overline{\mathbb{Q}}_{\ell}\right) \rightarrow \mathrm{D}_{c}^{b}\left(\mathfrak{X}_{s} \times_{s} \eta, \overline{\mathbb{Q}}_{\ell}\right)$ preserves $\sigma$-self-dual objects with respect to $K(-w)$.

Indeed, $\mathrm{R} \Psi$ is a right-lax symmetric monoidal functor. Hence, by Construction A.4.6, the composite $\mathrm{R} \Psi D_{\mathfrak{X}_{\eta}} \rightarrow D_{\mathrm{R} \Psi K_{\mathfrak{X}_{\eta}}} \mathrm{R} \Psi \rightarrow D_{\mathfrak{X}_{s}} \mathrm{R} \Psi$, which is a natural isomorphism (see [Illusie 1994, Théorème 4.2]), is symmetric.

Remark 2.1.4(6) can be applied to the exterior tensor power functor $(-)^{\bigotimes m}$ : $\mathrm{D}_{c}^{b}\left(X, \overline{\mathbb{Q}}_{\ell}\right) \rightarrow \mathrm{D}_{c}^{b}\left(X^{m}, \overline{\mathbb{Q}}_{\ell}\right), m \geq 0$. We now discuss a refinement

$$
\mathrm{D}_{c}^{b}\left(X, \overline{\mathbb{Q}}_{\ell}\right) \rightarrow \mathrm{D}_{c}^{b}\left(\left[X^{m} / \mathfrak{S}_{m}\right], \overline{\mathbb{Q}}_{\ell}\right), \quad A \mapsto A^{\otimes m},
$$

given by permutation. Readers not interested in this refinement may skip this part as it will not be used in the proofs of the results mentioned in the Introduction.

We briefly recall one way to define the symmetric product stack $\left[X^{m} / \mathfrak{S}_{m}\right]$. For every $k$-scheme $S$, the groupoid $\left[X^{m} / \mathfrak{S}_{m}\right](S)$ is the groupoid of pairs $(T, x)$, where $T$ is a finite étale cover of $S$ of degree $m$ and $x$ is an object of $X(T)$, with isomorphisms of pairs defined in the obvious way.

Remark 2.1.8. The functor (2-1-2) carries complexes $\sigma$-self-dual with respect to $K_{X}(-w)$ to complexes $\sigma^{m}$-self-dual with respect to $K_{\left[X^{m} / \mathfrak{S}_{m}\right]}(-m w)$.

Indeed, (- $)^{\unrhd m}$ is a symmetric monoidal functor. Hence, the isomorphism

$$
\left(D_{X}(-)\right)^{\unrhd m} \stackrel{\sim}{\longrightarrow} D_{K_{X}^{\bigotimes m}}\left((-)^{\bigotimes m}\right) \simeq D_{\left[X^{m} / \mathfrak{S}_{m}\right]}\left((-)^{\bigotimes m}\right)
$$

is symmetric by Construction A.4.6.

2.2. Symmetry of perverse sheaves. In this subsection, we study $\sigma$-self-dual perverse sheaves. We first prove a two-out-of-three property, which will play an important role in later sections. We then discuss a trichotomy for indecomposable perverse $\overline{\mathbb{Q}}_{\ell}$-sheaves. From this we deduce a criterion for semisimple perverse $\overline{\mathbb{Q}}_{\ell}$-sheaves to be $\sigma$-self-dual in terms of multiplicities of simple factors.

Proposition 2.2.1 (two-out-of-three). Let $A$ be a perverse $\overline{\mathbb{Q}}_{\ell}$-sheaf satisfying $A \simeq A^{\prime} \oplus A^{\prime \prime}$. If $A$ and $A^{\prime}$ are $\sigma$-self-dual with respect to $K_{X}(-w)$, then so is $A^{\prime \prime}$.

Proof. We write $D$ for $D_{K_{X}(-w)}$ and let $f: A \stackrel{\sim}{\longrightarrow} D A$ and $g^{\prime}: A^{\prime} \stackrel{\sim}{\longrightarrow} D A^{\prime}$ be $\sigma$-symmetric isomorphisms. We let $f^{\prime}: A^{\prime} \rightarrow D A^{\prime}$ denote the restriction of $f$ to $A^{\prime}$, namely the composite

$$
A^{\prime} \stackrel{i}{\longrightarrow} A \stackrel{f}{\sim} D A \stackrel{D i}{\longrightarrow} D A^{\prime},
$$

where $i: A^{\prime} \rightarrow A$ is the inclusion. Let $g: A \rightarrow D A$ be the direct sum of $g^{\prime}$ with the zero map $A^{\prime \prime} \rightarrow D A^{\prime \prime}$. These are $\sigma$-symmetric morphisms. Consider linear combinations $h_{a, b}=a f+b g$ and $h_{a, b}^{\prime}=a f^{\prime}+b g^{\prime}$, where $a, b \in \overline{\mathbb{Q}}_{\ell}$. By Lemma 2.1.3, there are only finitely many values of $(a: b)$ for which $h_{a, b}$ is not an isomorphism. The same holds for $h_{a, b}^{\prime}$. Therefore, there exist $a, b \in \overline{\mathbb{Q}}_{\ell}$ such that 
$h_{a, b}$ and $h_{a, b}^{\prime}$ are isomorphisms. Consider the orthogonal complement of $A^{\prime}$ in $A$ with respect to $h_{a, b}$ :

$$
B=\operatorname{Ker}\left(A \stackrel{h_{a, b}}{\sim} D A \stackrel{D i}{\longrightarrow} D A^{\prime}\right) .
$$

Then $h_{a, b}$ induces a $\sigma$-symmetric isomorphism $B \stackrel{\sim}{\longrightarrow} D B$. Moreover, $A \simeq A^{\prime} \oplus B$, so that $B \simeq A^{\prime \prime}$. Here we used the Krull-Schmidt theorem [Atiyah 1956, Theorem 1] and the fact that perverse sheaves have finite lengths.

Remark 2.2.2. The two-out-of-three property also holds more trivially for self-dual complexes. In fact, if we have decompositions of perverse $\overline{\mathbb{Q}}_{\ell}$-sheaves $A \simeq A^{\prime} \oplus A^{\prime \prime}$ and $B \simeq B^{\prime} \oplus B^{\prime \prime}$ such that $A \simeq D_{X} B(-w)$ and $A^{\prime} \simeq D_{X} B^{\prime}(-w)$, then we have $A^{\prime \prime} \simeq D_{X} B^{\prime \prime}(-w)$ by the Krull-Schmidt theorem.

Proposition 2.2.3 (trichotomy). Let $A$ be an indecomposable perverse $\overline{\mathbb{Q}}_{\ell}$-sheaf on $X$. Then exactly one of the following occurs:

- $A$ is 1-self-dual with respect to $K_{X}(-w)$;

- A is-1-self-dual with respect to $K_{X}(-w)$;

- A is not self-dual with respect to $K_{X}(-w)$.

This follows from general facts (Lemma A.2.7 and Remark A.2.8) applied to the category of perverse $\overline{\mathbb{Q}}_{\ell}$-sheaves.

Remark 2.2.4. In the case of a simple perverse $\overline{\mathbb{Q}}_{\ell}$-sheaf, the proof can be somewhat simplified with the help of Schur's lemma. This case is analogous to a standard result on complex representations of finite or compact groups [Serre 1998, Section 13.2, Proposition 38; Bröcker and tom Dieck 1995, Proposition II.6.5].

Remark 2.2.5. An indecomposable perverse $E_{\lambda}$-sheaf on $X$, self-dual with respect to $K_{X}(-w)$, is either 1-self-dual or -1 -self-dual, by Lemma A.2.7 and Remark A.2.8. Note that a simple perverse $E_{\lambda}$-sheaf can be 1-self-dual and -1-selfdual with respect to $K_{X}(-w)$ at the same time.

Corollary 2.2.6. Let $A \simeq \bigoplus_{B} B^{n_{B}}$ be a semisimple perverse $\overline{\mathbb{Q}}_{\ell}$-sheaf on $X$, where $B$ runs through isomorphism classes of simple perverse $\overline{\mathbb{Q}}_{\ell}$-sheaves on $X$. Then $A$ is $\sigma$-self-dual with respect to $K_{X}(-w)$ if and only if the following conditions hold:

(1) $n_{B}=n_{\left(D_{X} B\right)(-w)}$ for $B$ not self-dual with respect to $K_{X}(-w)$;

(2) $n_{B}$ is even for $B$ that are $-\sigma$-self-dual with respect to $K_{X}(-w)$.

Moreover, A is self-dual with respect to $K_{X}(-w)$ if and only if (1) holds.

In particular, if $B$ and $B^{\prime}$ are respectively 1-self-dual and -1-self-dual simple perverse sheaves on $X$, then $B \oplus B^{\prime}$ is self-dual but neither 1-self-dual nor -1-self-dual. 
Proof. It is clear that (1) is equivalent to the condition that $A$ is self-dual. If (1) and (2) hold, then $A$ is $\sigma$-self-dual by Proposition 2.2.3 and the fact that $B \oplus\left(D_{X} B\right)(-w)$ is $\sigma$-self-dual with respect to $K_{X}(-w)$ for all $B$ (Remark A.2.6(2)). It remains to show that if $A$ is $\sigma$-self-dual, then (2) holds. Let $B$ be $-\sigma$-self-dual. As $B^{\oplus n_{B}} \simeq B \otimes V$ is $\sigma$-self-dual, where $V=\overline{\mathbb{Q}}_{\ell}^{\oplus n_{B}}$, the isomorphism

$\operatorname{Hom}\left(B \otimes V, D_{X}(B \otimes V)(-w)\right)$

$$
\simeq \operatorname{Hom}\left(B,\left(D_{X} B\right)(-w)\right) \otimes \operatorname{Hom}\left(V, V^{*}\right) \simeq \operatorname{Hom}\left(V, V^{*}\right)
$$

provides a skew-symmetric $n_{B} \times n_{B}$ matrix with entries in $\overline{\mathbb{Q}}_{\ell}$, which implies that $n_{B}$ is even. More formally, we can apply the second part of Lemma A.3.9 to the fully faithful functor $F: V \mapsto B \otimes V$ from the category of finite-dimensional $\overline{\mathbb{Q}}_{\ell}$-vector spaces to $\mathrm{D}_{c}^{b}\left(X, \overline{\mathbb{Q}}_{\ell}\right)$. The natural isomorphism $F D \stackrel{\sim}{\longrightarrow} D_{K_{X}(-w)} F$ is $-\sigma$-self-dual.

Remark 2.2.7. The semisimplification of a $\sigma$-self-dual perverse sheaf is $\sigma$-self-dual by Lemma A.2.9. The converse does not hold. See Example 3.1.5 below.

We will need to consider more generally geometrically semisimple perverse sheaves, namely perverse sheaves whose pullbacks to $X_{\bar{k}}$ are semisimple. Part (1) of the following lemma extends [Beilinson et al. 1982, Corollaire 5.3.11] for pure perverse sheaves.

Lemma 2.2.8. Let $A$ be a geometrically semisimple perverse $\overline{\mathbb{Q}}_{\ell}$-sheaf on $X$.

(1) Let $i: Y \rightarrow X$ be a closed immersion with complementary open immersion $j: U \rightarrow X$. Then $A$ admits a unique decomposition $A \simeq j_{! *} j^{*} A \oplus i_{*} B$, where $B$ is a perverse sheaf on $Y$. Moreover, we have $B \simeq{ }^{p} \mathscr{H}^{0} i^{*} A \simeq{ }^{p} \mathrm{R}^{0} i^{!} A$.

(2) A admits a unique decomposition $A \simeq \bigoplus_{V} A_{V}$, where $V$ runs through irreducible closed substacks of $X$, and the support of each indecomposable direct summand of the perverse sheaf $A_{V}$ is $V$.

Assume additionally that $A$ is indecomposable. Then, by part (1) of the lemma, we have $j_{! *} j^{*} A \simeq A$ if $U$ intersects with the support of $A$ (and $j^{*} A=0$ otherwise). Moreover, the support of $A$ is irreducible, and $A$ is isomorphic to $f_{! *}(\mathscr{F}[d])$ for some immersion $f: W \rightarrow X$, with $W$ regular irreducible of dimension $d$, and some lisse $\overline{\mathbb{Q}}_{\ell}$-sheaf $\mathscr{F}$ on $V$.

Proof. (1) The proof is identical to that of [Beilinson et al. 1982, Corollaire 5.3.11]. The uniqueness of the decomposition is clear. For existence, it suffices to check that

- the adjunction map ${ }^{p} \mathscr{P}^{0} j ! j^{*} A \rightarrow A$ factorizes through the quotient $j_{! *} j^{*} A$ of ${ }^{p} \mathscr{H}^{0} j ! j^{*} A$, and the adjunction map $A \rightarrow{ }^{p} \mathrm{R}^{0} j_{*} j^{*} A$ factorizes through the subobject $j_{! *} j^{*} A$ of ${ }^{p} \mathrm{R}^{0} j_{*} j^{*} A$; 
- the composite of the adjunction maps $i_{*}{ }^{p} \mathrm{R}^{0} i^{!} A \rightarrow A \rightarrow i_{*}{ }^{p} \mathscr{H}^{0} i^{*} A$ is an isomorphism;

and these maps provide a decomposition of $A$. These statements can be easily checked over $\bar{k}$.

(2) Again the uniqueness is clear. The existence follows from the fact that the support of every indecomposable direct summand of $A$ is irreducible.

Remark 2.2.9. In Lemma 2.2.8, $A$ is $\sigma$-self-dual with respect to $K_{X}(-w)$ if and only if each direct summand $A_{V}$ in the support decomposition is $\sigma$-self-dual with respect to $K_{X}(-w)$.

As an application, we show that for geometrically semisimple perverse sheaves the property of being $\sigma$-self-dual is local for the Zariski topology. This Zariski local nature will be useful in Section 4.

Proposition 2.2.10. Let $\left(X_{\alpha}\right)_{\alpha \in I}$ be a Zariski open covering of $X$, and let $A$ and $B$ be geometrically semisimple perverse $\overline{\mathbb{Q}}_{\ell}$-sheaves on $X$. Then $A \simeq\left(D_{X} B\right)(-w)$ if and only if $A \mid X_{\alpha} \simeq\left(D_{X_{\alpha}} B \mid X_{\alpha}\right)(-w)$ for every $\alpha \in I$. Moreover, $A$ is $\sigma$-self-dual with respect to $K_{X}(-w)$ if and only if $A \mid X_{\alpha}$ is so with respect to $K_{X_{\alpha}}(-w)$ for every $\alpha \in I$.

Proof. We prove the second assertion, the proof of the first assertion being simpler. It suffices to show the "if" part. Let $j_{\alpha}: X_{\alpha} \rightarrow X$. By parts (5) and (8) of Remark 2.1.4, $j_{\alpha ! *} j_{\alpha}^{*} A$ is $\sigma$-self-dual. Since $j_{\alpha ! *} j_{\alpha}^{*} A \simeq \bigoplus_{V} A_{V}$, where $V$ satisfies $V \cap X_{\alpha} \neq \varnothing$, we conclude that each $A_{V}$ is $\sigma$-self-dual.

Alternatively we may apply Lemma 2.2.11 below. Indeed, by quasicompactness, we may assume that $I$ is finite. For $J \subseteq I$ nonempty, $j_{J ! *} j_{J}^{*} A$ is $\sigma$-self-dual. Thus the same holds for $A \simeq j_{\varnothing ! *} j_{\varnothing}^{*} A$ by the two-out-of-three property.

Lemma 2.2.11. Let $\left(X_{\alpha}\right)_{\alpha \in I}$ be a finite Zariski open covering of $X$, and let $A$ be a geometrically semisimple perverse $\overline{\mathbb{Q}}_{\ell}$-sheaf on $X$. Then

$$
\bigoplus_{\substack{J \subseteq I \\ \# J \text { even }}} j_{J ! *} j_{J}^{*} A \simeq \bigoplus_{\substack{J \subseteq I \\ \# J \text { odd }}} j_{J ! *} j_{J}^{*} A,
$$

where $j_{J}: \bigcap_{\alpha \in J} X_{\alpha} \rightarrow X$ is the open immersion.

Proof. We may assume that $A$ is indecomposable. Then both sides are direct sums of copies of $A$ and the multiplicities are equal:

$$
\sum_{\substack{0 \leq i \leq m \\
i \text { even }}}\left(\begin{array}{c}
m \\
i
\end{array}\right)=\sum_{\substack{0 \leq i \leq m \\
i \text { odd }}}\left(\begin{array}{c}
m \\
i
\end{array}\right) .
$$

Here $m \geq 1$ is the number of indices $\alpha \in I$ such that the support of $A$ intersects with $X_{\alpha}$. 


\section{Symmetry and decomposition of pure complexes}

In this section, we study symmetry of pure perverse sheaves and, more generally, of pure complexes that decompose into shifts of perverse sheaves. We first work over a finite field. In Section 3.1, we analyze $\sigma$-self-dual pure perverse sheaves and give a criterion in terms of multiplicities of factors. In Section 3.2, we study the behavior of such perverse sheaves under operations that preserve purity. The main result of this section is the preservation of a certain class of complexes under derived proper direct image (Theorem 3.2.3), which implies the finite field case of Theorem 1.8. Such preservation results constitute the starting point of the analysis in Section 4 of the effects of more general operations in the mixed case. In Section 3.3, we work over a separably closed base field and we prove preservation results for certain semisimple complexes, by reducing to the finite field case.

3.1. Symmetry of pure perverse sheaves over a finite field. In this subsection and the next, we work over a finite field $k=\mathbb{F}_{q}$. Recall that $X$, $Y$, etc., denote DeligneMumford stacks of finite presentation over $\mathbb{F}_{q}$. Let $\iota: \overline{\mathbb{Q}}_{\ell} \rightarrow \mathbb{C}$ be an embedding.

In this subsection, we study $\sigma$-self-dual $\iota$-pure perverse sheaves. We give a criterion for $\iota$-pure perverse sheaves to be $\sigma$-self-dual in terms of multiplicities of factors.

For $n \geq 1$, let $E_{n}$ be the sheaf on $\operatorname{Spec}\left(\mathbb{F}_{q}\right)$ of stalk $\left(\overline{\mathbb{Q}}_{\ell}\right)^{n}=\bigoplus_{i=1}^{n} \overline{\mathbb{Q}}_{\ell} e_{i}$ on which Frobenius $F=$ Frob $_{q}$ acts unipotently with one Jordan block: $F e_{1}=e_{1}$ and $F e_{i}=e_{i}+e_{i-1}$ for $i>1$. Recall that any indecomposable $\iota$-pure perverse sheaf $A$ on $X$ is isomorphic to a perverse sheaf of the form $B \otimes a_{X}^{*} E_{n}$, where $B$ is a simple perverse sheaf on $X, n \geq 1$, and where $a_{X}: X \rightarrow \operatorname{Spec}\left(\mathbb{F}_{q}\right)$.

Proposition 3.1.1. Let $w \in \mathbb{Z}$, and let $A$ be a perverse $\overline{\mathbb{Q}}_{\ell}$-sheaf on $X$, isomorphic to $\bigoplus_{B}\left(B \otimes a_{X}^{*} E_{n}\right)^{m_{B, n}}$, where $B$ runs over simple perverse $\overline{\mathbb{Q}}_{\ell}$-sheaves on $X$. Then $A$ is $\sigma$-self-dual with respect to $K_{X}(-w)$ if and only if the following conditions hold:

(1) $m_{B, n}=m_{\left(D_{X} B\right)(-w), n}$ for $B$ not self-dual with respect to $K_{X}(-w)$;

(2) $m_{B, n}$ is even for $B$-self-dual with respect to $K_{X}(-w)$ and $n$ even;

(3) $m_{B, n}$ is even for $B-\sigma$-self-dual with respect to $K_{X}(-w)$ and $n$ odd.

Moreover, A is self-dual with respect to $K_{X}(-w)$ if and only if (1) holds.

Proof. The equivalence between (1) and the condition that $A$ is self-dual follows from the isomorphism $D_{X}\left(B \otimes a_{X}^{*} E_{n}\right) \simeq D_{X} B \otimes a_{X}^{*} E_{n}$. For the "if" part of the $\sigma$-selfdual case, note that $C \oplus D_{X} C(-w)$ is $\sigma$-self dual with respect to $K_{X}(-w)$, so that, by the trichotomy in Proposition 2.2.3, it suffices to show that $B \otimes a_{X}^{*} E_{n}$ is $\sigma^{\prime}$-self-dual (resp. $-\sigma^{\prime}$-self-dual) for $B \sigma^{\prime}$-self-dual and $n$ odd (resp. even). For the "only if" part, we reduce to the case where $m_{B, n}=0$ for all $B$ except for one $\sigma^{\prime}$-self-dual $B$. For both parts, consider the functor $F=B \otimes a_{X}^{*}-: \operatorname{Perv}\left(\operatorname{Spec}\left(\mathbb{F}_{q}\right), \overline{\mathbb{Q}}_{\ell}\right) \rightarrow \operatorname{Perv}\left(X, \overline{\mathbb{Q}}_{\ell}\right)$, which is fully faithful by Lemma 3.1.2 below. The natural isomorphism $F D_{\operatorname{Spec}\left(\mathbb{F}_{q}\right)} \simeq$ 
$D_{K_{X}(-w)} F$ is $\sigma^{\prime}$-symmetric. By Lemma A.3.8, we are then reduced to the case where $X=\operatorname{Spec}\left(\mathbb{F}_{q}\right)$ and $B=\left(\overline{\mathbb{Q}}_{\ell}\right)_{X}$, which follows from Lemma 3.1.3 below.

Lemma 3.1.2. Let $B$ be a simple perverse $\overline{\mathbb{Q}}_{\ell}$-sheaf on $X$. Then the functor $B \otimes a_{X}^{*}-i$ fully faithful. In other words, for $\overline{\mathbb{Q}}_{\ell}$-sheaves $E$ and $E^{\prime}$ on $\operatorname{Spec}\left(\mathbb{F}_{q}\right)$, the map $\alpha: \operatorname{Hom}\left(E, E^{\prime}\right) \rightarrow \operatorname{Hom}\left(B \otimes a_{X}^{*} E, B \otimes a_{X}^{*} E^{\prime}\right)$ is an isomorphism.

Proof. We have $B \simeq j_{! *}(\mathscr{F}[d])$, where $j: U \rightarrow X$ is an immersion, with $U_{\text {red }}$ connected regular, purely of dimension $d$, and where $\mathscr{F}$ is a simple lisse $\overline{\mathbb{Q}}_{\ell}$-sheaf on $U$. We have $B \otimes a_{X}^{*} E \simeq j_{! *}\left(\left(\mathscr{F} \otimes a_{U}^{*} E\right)[d]\right)$. The map $\alpha$ is the composite

$$
\operatorname{Hom}\left(E, E^{\prime}\right) \stackrel{\beta}{\longrightarrow} \operatorname{Hom}\left(\mathscr{F} \otimes a_{U}^{*} E, \mathscr{F} \otimes a_{U}^{*} E^{\prime}\right) \stackrel{\gamma}{\longrightarrow} \operatorname{Hom}\left(B \otimes a_{X}^{*} E, B \otimes a_{X}^{*} E^{\prime}\right),
$$

where $\gamma$ is an isomorphism by Lemma 2.1.5. The map $\beta$ is obviously injective. To show that $\beta$ is an isomorphism, we may assume that $E=E_{n}, E^{\prime}=E_{m}$. Since the socle of $\mathscr{F} \otimes a_{U}^{*} E_{m}$ is $\mathscr{F}$,

$$
\operatorname{Hom}(\mathscr{F}, \mathscr{F}) \simeq \operatorname{Hom}\left(\mathscr{F}, \mathscr{F} \otimes a_{U}^{*} E_{m}\right)
$$

is one-dimensional. Dually, since the cosocle of $\mathscr{F} \otimes a_{U}^{*} E_{n}$ is $\mathscr{F}$,

$$
\operatorname{Hom}\left(\mathscr{F} \otimes a_{U}^{*} E_{n}, \mathscr{F}\right) \simeq \operatorname{Hom}(\mathscr{F}, \mathscr{F})
$$

is one-dimensional. Thus

$$
\operatorname{dim} \operatorname{Hom}\left(\mathscr{F} \otimes a_{U}^{*} E_{n}, \mathscr{F} \otimes a_{U}^{*} E_{m}\right) \leq \min \{n, m\}=\operatorname{dim} \operatorname{Hom}\left(E_{n}, E_{m}\right) .
$$

It follows that $\beta$ is an isomorphism.

Lemma 3.1.3. Let $L$ be a field of characteristic $\neq 2$, and let $N_{n} \in \mathrm{M}_{n \times n}(L)$ be the matrix defined by $\left(N_{n}\right)_{i, j}=1$ for $i=j-1$ and $\left(N_{n}\right)_{i, j}=0$ otherwise. Let $m_{1}, \ldots, m_{l} \geq 0$ be integers, and let

$$
N:=N\left(m_{1}, \ldots, m_{l}\right):=\operatorname{diag}\left(N_{1}, \ldots, N_{1}, \ldots, N_{l}, \ldots, N_{l}\right),
$$

where each $N_{n}$ is repeated $m_{n}$ times. Then there exists an invertible symmetric (resp. invertible skew-symmetric) matrix A such that $A N=-N^{T} A$ if and only if $m_{n}$ is even for $n$ even (resp. $m_{n}$ is even for $n$ odd).

For $L$ of characteristic 0 , the equality is equivalent to $\exp (N)^{T} A \exp (N)=A$. Proof. We denote the entries of $A$ by $a_{n, c, i}^{n^{\prime}, c^{\prime}, i^{\prime}}$, where

$$
1 \leq n, n^{\prime} \leq l, \quad 1 \leq c \leq m_{n}, \quad 1 \leq c^{\prime} \leq m_{n^{\prime}}, \quad 1 \leq i \leq n, \quad 1 \leq i^{\prime} \leq n^{\prime} .
$$

Then we have $A N=-N^{T} A$ if and only if $a_{n, c, i}^{n^{\prime}, c^{\prime}, i^{\prime}}=-a_{n, c, i-1}^{n^{\prime}, c^{\prime}, i^{\prime}+1}$ for $1<i \leq n$, $1 \leq i^{\prime}<n^{\prime}$ and $a_{n, c, i}^{n^{\prime}, c^{\prime}, i^{\prime}}=0$ for $i+i^{\prime} \leq \max \left\{n, n^{\prime}\right\}$. Let $A_{n}$ be the $n \times n$ matrix given by $\left(A_{n}\right)_{i j}=(-1)^{i}$ for $i+j=n+1$ and $\left(A_{n}\right)_{i j}=0$ otherwise. For $N=N_{n}, n$ odd (resp. even), we can take $A=A_{n}$. For $N=\operatorname{diag}\left(N_{n}, N_{n}\right), n$ even (resp. odd), we 
can take $A=\left(\begin{array}{cc}0 & A_{n} \\ -A_{n} & 0\end{array}\right)$. The "if" part follows. The "only if" part follows from the case $\sigma=-1$ of Proposition A.6.8, because $P_{1-n}$ is $(-1)^{1-n}$-self-dual (resp. $(-1)^{n}$-self-dual) and $\operatorname{dim} P_{1-n}$ equals $m_{n}$. Let us give a more elementary proof of the "only if" part by induction on $l$. For $l=1$, the assertion is void (resp. $A$ defines a nondegenerate alternating bilinear form on an $m_{1}$-dimensional vector space, which implies that $m_{1}$ is even). For $l \geq 2$, consider the $m_{l} \times m_{l}$ submatrices $B=\left(a_{l, c, 1}^{l, c^{\prime}, l}\right)_{c, c^{\prime}}$, $C=\left(a_{l, c, l}^{l, c^{\prime}, 1}\right)_{c, c^{\prime}}$ of $A$. Let $A^{\prime}$ be the matrix obtained from $A$ by removing the rows and columns in $A$ that contain entries of $B$ or $C$. Note that for $i^{\prime}<l$ we have $a_{l, c, 1}^{n^{\prime}, c^{\prime}, i^{\prime}}=0$, and for $i<l$ we have $a_{n, c, i}^{l, c^{\prime}, 1}=0$. Thus, up to reordering the indices, we have

$$
A=\left(\begin{array}{ccc}
A^{\prime} & 0 & B^{\prime} \\
0 & 0 & B \\
C^{\prime} & C & D
\end{array}\right) .
$$

It follows that $B^{T}=\sigma^{\prime} C=(-1)^{l-1} \sigma^{\prime} B$, where $\sigma^{\prime}=1$ (resp. $\sigma^{\prime}=-1$ ), and that $B$ is invertible, so that $m_{l}$ is even for $l$ even (resp. $l$ odd). Moreover, $A^{\prime}$ is invertible symmetric (resp. invertible skew-symmetric) and $A^{\prime} N^{\prime}=-N^{\prime T} A^{\prime}$, where $N^{\prime}=N\left(m_{1}, \ldots, m_{l-3}, m_{l-2}+m_{l}, m_{l-1}\right)\left(N^{\prime}=N\left(m_{1}\right)\right.$ for $\left.l=2\right)$. The assertion then follows from the induction hypothesis.

Example 3.1.4. Let $A$ and $A^{\prime}$ be sheaves on $X=\operatorname{Spec}\left(\mathbb{F}_{q}\right)$ and let $w \in \mathbb{Z}$. For $n \geq 1$ and $\lambda \in \overline{\mathbb{Q}}_{\ell}^{\times}$, we let $\mu_{\lambda, n}$ and $\mu_{\lambda, n}^{\prime}$ denote the number of $n \times n$ Jordan blocks of eigenvalue $\lambda$ in the Jordan normal forms of the Frobenius Frob $q$ acting on $A_{\overline{\mathbb{F}}_{q}}$ and $\left(A^{\prime}\right)_{\overline{\mathbb{F}}_{q}}$, respectively. Then:

- $A \simeq\left(D_{X} A^{\prime}\right)(-w)$ if and only if $\mu_{\lambda, n}=\mu_{q^{w} / \lambda, n}^{\prime}$ for all $n \geq 1$ and all $\lambda$. In particular, $A$ is self-dual with respect to $\overline{\mathbb{Q}}_{\ell}(-w)$ if and only if $\mu_{\lambda, n}=\mu_{q^{w} / \lambda, n}$ for all $n \geq 1$ and all $\lambda$. Note that the last condition trivially holds for $\lambda= \pm q^{w / 2}$.

- $A$ is 1 -self-dual (resp. -1 -self-dual) with respect to $\overline{\mathbb{Q}}_{\ell}(-w)$ if and only if it is self-dual with respect to $\overline{\mathbb{Q}}_{\ell}(-w)$ and $\mu_{q^{w / 2}, n}, \mu_{-q^{w / 2}, n}$ are even for $n$ even (resp. $n$ odd).

Example 3.1.5. Let $B$ be a simple perverse sheaf $\iota$-pure of weight $w$, not self-dual with respect to $K_{X}(-w)$. Then $A=B^{\oplus 2} \oplus\left(\left(D_{X} B\right)(-w) \otimes a_{X}^{*} E_{2}\right)$ is not self-dual, but the semisimplification of $A$ is both 1-self-dual and -1-self-dual.

Remark 3.1.6. (1) An $\iota$-pure complex self-dual with respect to $K_{X}(-w)$ is necessarily of weight $w$.

(2) Every simple perverse $\overline{\mathbb{Q}}_{\ell}$-sheaf is $\iota$-pure by a theorem of Lafforgue [2002, Corollaire VII.8] (with a gap filled by Deligne [2012, Théorème 1.6]; see [Sun 2012b, Remark 2.8.1] for the case of stacks).

(3) The two-out-of-three property (Proposition 2.2.1) in the case of $\iota$-pure perverse sheaves also follows from the criterion of Proposition 3.1.1. 
(4) Since $\iota$-pure perverse sheaves are geometrically semisimple, for such perverse sheaves the property of being self-dual (resp. $\sigma$-self-dual) is local for the Zariski topology by Proposition 2.2.10.

3.2. Symmetry and decomposition of pure complexes over a finite field. In this subsection, we study the behavior of $\sigma$-self-dual $\iota$-pure perverse sheaves under operations that preserve purity. The main goal is to prove the finite field case of Theorem 1.8 on derived proper direct image of $\sigma$-self-dual $\iota$-pure perverse sheaves. The behavior of $\sigma$-self-dual complexes has already been described in Section 2.1. The focus of this subsection is on decomposition and on the self-duality of individual perverse cohomology sheaves. To state our results, it is convenient to introduce the following terminology.

Definition 3.2.1 (split complexes). We say that a complex of $\overline{\mathbb{Q}}_{\ell}$-sheaves $A$ is split if it is a direct sum of shifts of perverse sheaves, or, in other words, if $A \simeq \bigoplus_{i}\left(\mathscr{H}^{i} A\right)[-i]$.

Definition 3.2.2 $\left(\mathrm{D}_{l, \sigma}^{w}\right)$. Let $w \in \mathbb{Z}$. Denote by $\mathrm{D}_{\iota, \sigma}^{w}\left(X, \overline{\mathbb{Q}}_{\ell}\right) \subseteq \mathrm{Ob}\left(\mathrm{D}_{c}^{b}\left(X, \overline{\mathbb{Q}}_{\ell}\right)\right)$ (resp. $\left.\mathrm{D}_{\iota, \mathrm{sd}}^{w}\left(X, \overline{\mathbb{Q}}_{\ell}\right) \subseteq \mathrm{Ob}\left(\mathrm{D}_{c}^{b}\left(X, \overline{\mathbb{Q}}_{\ell}\right)\right)\right)$ the subset consisting of split $\iota$-pure complexes $A$ of weight $w$ such that ${ }^{p} \mathscr{H}^{i} A$ is $(-1)^{w+i} \sigma$-self-dual (resp. self-dual) with respect to $K_{X}(-w-i)$ for all $i$. Denote by $\mathrm{D}_{\iota, \mathrm{d}}^{w}\left(X, \overline{\mathbb{Q}}_{\ell}\right) \subseteq \mathrm{Ob}\left(\mathrm{D}_{c}^{b}\left(X, \overline{\mathbb{Q}}_{\ell}\right) \times \mathrm{D}_{c}^{b}\left(X, \overline{\mathbb{Q}}_{\ell}\right)\right)$ the subset consisting of pairs $(A, B)$ of split $\iota$-pure complexes of weight $w$ such that $p_{\mathscr{H}^{i}} A$ is isomorphic to $\left(D_{X}{ }^{p} \mathscr{H}^{i} B\right)(-w-i)$ for all $i$.

By definition, we have $\mathrm{D}_{\iota, \mathrm{sd}}^{w}\left(X, \overline{\mathbb{Q}}_{\ell}\right)=\Delta^{-1}\left(\mathrm{D}_{\iota, \mathrm{d}}^{w}\left(X, \overline{\mathbb{Q}}_{\ell}\right)\right)$, where $\Delta: \mathrm{D}_{c}^{b}\left(X, \overline{\mathbb{Q}}_{\ell}\right) \rightarrow$ $\mathrm{D}_{c}^{b}\left(X, \overline{\mathbb{Q}}_{\ell}\right) \times \mathrm{D}_{c}^{b}\left(X, \overline{\mathbb{Q}}_{\ell}\right)$ is the diagonal embedding.

Since in this subsection we will only consider operations that preserve purity, the factor $(-1)^{w}$ in the definition above is fixed, hence not essential. We include this factor here to make the definition compatible with the mixed case studied in Section 4, where the factor is essential (see Definition 4.2.1).

The main result of this section is the following preservation result under proper direct image, which clearly implies the finite field case of Theorem 1.8.

Theorem 3.2.3. Let $f: X \rightarrow Y$ be a proper morphism of Deligne-Mumford stacks of finite presentation over $\mathbb{F}_{q}$, where $Y$ has finite inertia. Then $\mathrm{R} f_{*}$ preserves $\mathrm{D}_{\iota, \sigma}^{w}$ and $\mathrm{D}_{\iota, \mathrm{d}}^{w}$. In other words, for $A \in \mathrm{D}_{\iota, \sigma}^{w}\left(X, \overline{\mathbb{Q}}_{\ell}\right)$ we have $\mathrm{R} f_{*} A \in \mathrm{D}_{\iota, \sigma}^{w}\left(Y, \overline{\mathbb{Q}}_{\ell}\right)$, and for $(A, B) \in \mathrm{D}_{\iota, \mathrm{d}}^{w}\left(X, \overline{\mathbb{Q}}_{\ell}\right)$ we have $\left(\mathrm{R} f_{*} A, \mathrm{R} f_{*} B\right) \in \mathrm{D}_{\iota, \mathrm{d}}^{w}$.

The preservation of $\mathrm{D}_{\iota, \mathrm{d}}^{w}$ has the following two consequences, obtained respectively by considering the diagonal embedding and the first factor.

Corollary 3.2.4. Let $f: X \rightarrow Y$ be a proper morphism, where $Y$ has finite inertia. Then $\mathrm{R} f_{*}$ preserves $\mathrm{D}_{\iota, \mathrm{sd}}^{w}$. 
Corollary 3.2.5. Let $f: X \rightarrow Y$ be a proper morphism, where $Y$ has finite inertia. Then $\mathrm{R} f_{*}$ preserves split ı-pure complexes of weight $w$. In other words, if $A$ is a split ı-pure complex of weight $w$ on $X$, then $\mathrm{R} f_{*} A$ is a split ı-pure complex of weight $w$ on $Y$.

Corollary 3.2.5 clearly extends to the case where $w \in \mathbb{R}$. Recall that the Beilinson-Bernstein-Deligne-Gabber decomposition theorem [Beilinson et al. 1982, Théorème 5.4.5] ([Sun 2012a, Theorem 1.2] for the case of stacks) implies that the pullback of $\mathrm{R} f_{*} A$ (or any $\iota$-pure complex on $Y$ ) to $Y \otimes_{\mathbb{F}_{q}} \overline{\mathbb{F}}_{q}$ is split.

Remark 3.2.6. For $w \in \mathbb{Z}$, let $\mathrm{D}_{\iota, \mathrm{spl}}^{w}\left(X, \overline{\mathbb{Q}}_{\ell}\right) \subseteq \mathrm{D}_{c}^{b}\left(X, \overline{\mathbb{Q}}_{\ell}\right)$ be the full subcategory consisting of split $\iota$-pure complexes of weight $w$. Consider the twisted dualizing functor $\bar{D}_{\iota, X}^{w}: \mathrm{D}_{\iota, \mathrm{spl}}^{w}\left(X, \overline{\mathbb{Q}}_{\ell}\right)^{\mathrm{op}} \rightarrow \mathrm{D}_{\iota, \mathrm{spl}}^{w}\left(X, \overline{\mathbb{Q}}_{\ell}\right)$ that carries each complex $A$ to $\bigoplus_{i}\left(D_{X} p_{\mathscr{H}^{i}} A\right)(-w-i)[-i]$. Then $\mathrm{D}_{\iota, \mathrm{d}}^{w}$ is the collection of pairs $\left(A, \bar{D}_{\iota, X}^{w} A\right)$. Thus the preservation of $\mathrm{D}_{\iota, \mathrm{d}}^{w}$ by $\mathrm{R} f_{*}$ is equivalent to the preservation of $\mathrm{D}_{\iota, \mathrm{spl}}^{w}$ and the existence of an isomorphism $\mathrm{R} f_{*} \bar{D}_{\iota, X}^{w} A \simeq \bar{D}_{\iota, X}^{w} \mathrm{R} f_{*} A$ for every object $A$ of $\mathrm{D}_{\iota, \mathrm{spl}}^{w}\left(X, \overline{\mathbb{Q}}_{\ell}\right)$. Our proof of Theorem 3.2.3 relies on the two-out-of-three property, and the resulting isomorphism is not necessarily functorial in $A$. Thus our proof does not provide a natural isomorphism between the functors $\mathrm{R} f_{*} \bar{D}_{\iota, X}^{w}$ and $\bar{D}_{\iota, X}^{w} \mathrm{R} f_{*}$.

Let us first recall that the following operations preserve $\iota$-pure complexes [Beilinson et al. 1982, Stabilités 5.1.14, Corollaire 5.4.3] ([Sun 2012a] for the case of stacks). The proof makes use of the fact that these operations commute with duality (up to shift and twist).

Remark 3.2.7 (preservation of $\iota$-pure complexes). Let $f: X \rightarrow Y$ be a morphism, and let $w, w^{\prime} \in \mathbb{R}$.

(1) For $A \in \mathrm{D}_{c}^{b}\left(X, \overline{\mathbb{Q}}_{\ell}\right) \iota$-pure of weight $w, A(n)$ is $\iota$-pure of weight $w-2 n$ and $A[n]$ is $\iota$-pure of weight $w+n$ for $n \in \mathbb{Z}$.

(2) $A \in \mathrm{D}_{c}^{b}\left(X, \overline{\mathbb{Q}}_{\ell}\right)$ is $\iota$-pure of weight $w$ if and only if $D_{X} A$ is $\iota$-pure of weight $-w$.

(3) If $f$ is an open immersion, the functor $f_{\text {!* }}$ preserves $\iota$-pure perverse sheaves of weight $w$.

(4) Assume that $f$ is smooth. Then $f^{*}$ preserves $\iota$-pure complexes of weight $w$. Moreover, if $f$ is surjective, then $A \in \mathrm{D}_{c}^{b}\left(Y, \overline{\mathbb{Q}}_{\ell}\right)$ is $\iota$-pure of weight $w$ if and only if $f^{*} A$ is so.

(5) Assume that $X$ and $Y$ are regular. Then $f^{*}$ preserves $\iota$-pure complexes of weight $w$ in $\mathrm{D}_{\text {lisse }}^{b}$. Moreover, if $f$ is surjective, then $A \in \mathrm{D}_{\text {lisse }}^{b}\left(Y, \overline{\mathbb{Q}}_{\ell}\right)$ is $\iota$-pure of weight $w$ if and only if $f^{*} A$ is so.

(6) For $A \in \mathrm{D}_{c}^{b}\left(X, \overline{\mathbb{Q}}_{\ell}\right) \iota$-pure of weight $w$ and $A^{\prime} \in \mathrm{D}_{c}^{b}\left(X^{\prime}, \overline{\mathbb{Q}}_{\ell}\right) \iota$-pure of weight $w^{\prime}$, $A \otimes A^{\prime} \in \mathrm{D}_{c}^{b}\left(X \times X^{\prime}, \overline{\mathbb{Q}}_{\ell}\right)$ is $\iota$-pure of weight $w+w^{\prime}$. 
(7) $A \in \mathrm{D}_{c}^{b}\left(X, \overline{\mathbb{Q}}_{\ell}\right)$ is $\iota$-pure of weight $w$ if and only if $A^{\bigotimes m} \in \mathrm{D}_{c}^{b}\left(\left[X^{m} / \mathfrak{S}_{m}\right], \overline{\mathbb{Q}}_{\ell}\right)$ is $\iota$-pure of weight $m w$, where $m \geq 1$.

(8) If $f$ is a proper morphism, $\mathrm{R} f_{*}$ preserves $\iota$-pure complexes of weight $w$.

(9) Assume that $f$ is a closed immersion and let $A \in \mathrm{D}_{c}^{b}\left(X, \overline{\mathbb{Q}}_{\ell}\right)$. Then $A$ is $\iota$-pure of weight $w$ if and only if $f_{*} A$ is so.

Recall that these operations also preserve $\sigma$-self-dual complexes (Remark 2.1.4). With the exception of derived proper direct image, the operations also preserve perversity (up to shift). Hence, they also preserve $\mathrm{D}_{l, \sigma}^{w}$, up to modification of $w$ and $\sigma$. The details are given below. The case of $(-)^{\bigotimes m}$ requires some additional arguments and will be given in Proposition 3.2.14 later.

Remark 3.2.8 (preservation of $\mathrm{D}_{\iota, \sigma}^{w}$, easy part). Let $f: X \rightarrow Y$ be a morphism, and let $w, w^{\prime} \in \mathbb{Z}$.

(1) If $A \in \mathrm{D}_{\iota, \sigma}^{w}\left(X, \overline{\mathbb{Q}}_{\ell}\right)$, then $A[n] \in \mathrm{D}_{\iota, \sigma}^{w+n}\left(X, \overline{\mathbb{Q}}_{\ell}\right)$ and $A(n) \in \mathrm{D}_{\iota, \sigma}^{w-2 n}\left(X, \overline{\mathbb{Q}}_{\ell}\right)$ for $n \in \mathbb{Z}$.

(2) $D_{X}$ carries $\mathrm{D}_{\iota, \sigma}^{w}\left(X, \overline{\mathbb{Q}}_{\ell}\right)$ to $\mathrm{D}_{\iota, \sigma}^{-w}\left(X, \overline{\mathbb{Q}}_{\ell}\right)$.

(3) Assume that $X$ is regular and let $\mathscr{F}$ be a lisse $\overline{\mathbb{Q}}_{\ell}$-sheaf on $X$, punctually $\iota$-pure of weight $w$. Then there exists a nondegenerate $\sigma$-symmetric pairing $\mathscr{F} \otimes \mathscr{F} \rightarrow \overline{\mathbb{Q}}_{\ell}(-w)$ if and only if $\mathscr{F}$ belongs to $\mathrm{D}_{\iota,(-1)^{w} \sigma}^{w}\left(X, \overline{\mathbb{Q}}_{\ell}\right)$.

(4) If $f$ is smooth, then $f^{*}$ preserves $\mathrm{D}_{\iota, \sigma}^{w}$.

(5) If $X$ and $Y$ are regular, then $f^{*}$ preserves $\mathrm{D}_{\iota, \sigma}^{w} \cap \mathrm{D}_{\text {lisse }}^{b}$.

(6) The functor $-\otimes-$ carries $\mathrm{D}_{\iota, \sigma}^{w}\left(X, \overline{\mathbb{Q}}_{\ell}\right) \times \mathrm{D}_{\iota, \sigma^{\prime}}^{w^{\prime}}\left(X^{\prime}, \overline{\mathbb{Q}}_{\ell}\right)$ to $\mathrm{D}_{\iota, \sigma \sigma^{\prime}}^{w+w^{\prime}}\left(X \times X^{\prime}, \overline{\mathbb{Q}}_{\ell}\right)$.

(7) Assume that $f$ is a closed immersion and let $A \in \mathrm{D}_{c}^{b}\left(X, \overline{\mathbb{Q}}_{\ell}\right)$. Then we have $A \in \mathrm{D}_{\iota, \sigma}^{w}\left(X, \overline{\mathbb{Q}}_{\ell}\right)$ if and only if $f_{*} A \in \mathrm{D}_{\iota, \sigma}^{w}\left(Y, \overline{\mathbb{Q}}_{\ell}\right)$.

(8) Assume that $f$ is an open immersion and let $A \in \operatorname{Perv}\left(X, \overline{\mathbb{Q}}_{\ell}\right)$. Then we have $A \in \mathrm{D}_{\iota, \sigma}^{w}\left(X, \overline{\mathbb{Q}}_{\ell}\right)$ if and only if $f_{! *} A \in \mathrm{D}_{\iota, \sigma}^{w}\left(Y, \overline{\mathbb{Q}}_{\ell}\right)$.

Similar properties hold for $\mathrm{D}_{\iota, \mathrm{d}}^{w}$.

Next we transcribe the two-out-of-three property (Proposition 2.2.1) established earlier in terms of $\mathrm{D}_{\iota, \sigma}^{w}$.

Remark 3.2.9. If $A, A^{\prime}, A^{\prime \prime} \in \mathrm{D}_{c}^{b}\left(X, \overline{\mathbb{Q}}_{\ell}\right)$ satisfy $A \simeq A^{\prime} \oplus A^{\prime \prime}$ and two of the three complexes are in $\mathrm{D}_{\iota, \sigma}^{w}$, then so is the third one. A similar property holds for $\mathrm{D}_{\iota, \mathrm{d}}$.

Note that the proper direct image $\mathrm{R} f_{*}$ does not preserve perversity and in general there seems to be no canonical way to produce pairings on the perverse cohomology sheaves ${ }^{p} \mathrm{R}^{i} f_{*} A$ from pairings on $A$. In the case of projective direct image, the relative hard Lefschetz theorem provides such pairings. Let us first fix some terminology on projective morphisms of Deligne-Mumford stacks. 
Definition 3.2.10. Let $f: X \rightarrow Y$ be a quasicompact schematic morphism of Deligne-Mumford stacks. We say that an invertible sheaf $\mathscr{L}$ on $X$ is $f$-ample if, for one (or, equivalently, for every) étale surjective morphism $g: Y^{\prime} \rightarrow Y$ where $Y^{\prime}$ is a scheme, $h^{*} \mathscr{L}$ is $f^{\prime}$-ample [Grothendieck 1961, Définition 4.6.1]. Here $h$ and $f^{\prime}$ are as shown in the following Cartesian square:

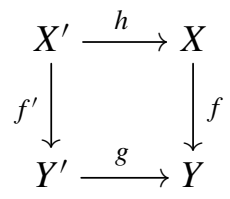

We say that a morphism $f: X \rightarrow Y$ of Deligne-Mumford stacks is quasiprojective if it is schematic, of finite presentation, and if there exists an $f$-ample invertible sheaf on $X$. We say that $f$ is projective if it is quasiprojective and proper.

The following is an immediate extension of the case of schemes [Beilinson et al. 1982, Théorème 5.4.10].

Proposition 3.2.11 (relative hard Lefschetz). Let $f: X \rightarrow Y$ be a projective morphism of Deligne-Mumford stacks of finite presentation over $\mathbb{F}_{q}$. Let $\eta \in \mathrm{H}^{2}\left(X, \overline{\mathbb{Q}}_{\ell}(1)\right)$ be the first Chern class of an f-ample invertible sheaf on $X$. Let $A$ be an ı-pure perverse sheaf on $X$. Then, for $i \geq 0$, the morphism

$$
{ }^{p} \mathrm{R}^{-i} f_{*}\left(\eta^{i} \otimes \mathrm{id}_{A}\right):{ }^{p} \mathrm{R}^{-i} f_{*} A \rightarrow{ }^{p} \mathrm{R}^{i} f_{*} A(i)
$$

is an isomorphism.

By Deligne's decomposition theorem [1994], the proposition implies that $\mathrm{R} f_{*} A$ is split.

Proposition 3.2.12. Let $f: X \rightarrow Y$ be a projective morphism, and let $w \in \mathbb{Z}$. Then $\mathrm{R} f_{*}$ preserves $\mathrm{D}_{\iota, \sigma}^{w}$ and $\mathrm{D}_{\iota, \mathrm{d}}^{w}$.

Proof. We prove the case of $\mathrm{D}_{\iota, \sigma}^{w}$, the case of $\mathrm{D}_{\iota, \mathrm{d}}^{w}$ being simpler. It suffices to show that, for every $(-1)^{w} \sigma$-self-dual $\iota$-pure perverse sheaf $A$ of weight $w, \mathrm{R} f_{*} A$ belongs to $\mathrm{D}_{\iota, \sigma}^{w}$. Given a $(-1)^{w} \sigma$-symmetric isomorphism $A \stackrel{\sim}{\longrightarrow}\left(D_{X} A\right)(-w)$, the isomorphism

${ }^{p} \mathrm{R}^{-i} f_{*} A \stackrel{\sim}{\longrightarrow}{ }^{p} \mathrm{R}^{-i} f_{*}\left(D_{X} A\right)(-w) \underset{\eta^{i}}{\sim}{ }^{p} \mathrm{R}^{i} f_{*}\left(D_{X} A\right)(i-w) \stackrel{\sim}{\longrightarrow}\left(D_{Y}{ }^{p} \mathrm{R}^{-i} f_{*} A\right)(i-w)$

corresponding to the pairing obtained from

$$
\begin{aligned}
\mathrm{R} f_{*} A[-i] \otimes \mathrm{R} f_{*} A[-i] & \rightarrow \mathrm{R} f_{*}(A \otimes A)[-2 i] \\
& \rightarrow \mathrm{R} f_{*} K_{X}(-w)[-2 i] \stackrel{\eta^{i}}{\longrightarrow} \mathrm{R} f_{*} K_{X}(i-w) \rightarrow K_{Y}(i-w)
\end{aligned}
$$

is $(-1)^{w+i} \sigma$-symmetric by Lemma A.5.11. 
Proof of Theorem 3.2.3. We will prove the case of $\mathrm{D}_{\iota, \sigma}^{w}$. The case of $\mathrm{D}_{\iota, \mathrm{d}}^{w}$ is similar. Consider the diagram

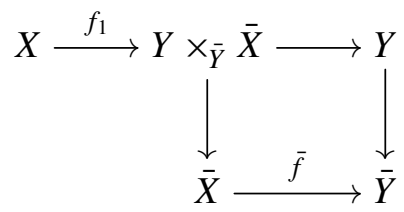

in which $\bar{f}$ is the morphism of coarse moduli spaces (they exist by the Keel-Mori theorem [1997]) associated to $f$. Since $f_{1}$ is proper and quasifinite, $\mathrm{R} f_{1 *}$ is $t$-exact for the perverse $t$-structures, and by Remarks 3.2.7(8) and 2.1.4(3) we see that the theorem holds for $f_{1}$. Thus we may assume that $f$ is representable. We proceed by induction on the dimension of $X$. Let $A \in \mathrm{D}_{\iota, \sigma}^{w}\left(X, \overline{\mathbb{Q}}_{\ell}\right)$; we may assume that $A$ is perverse. Applying Chow's lemma [Raynaud and Gruson 1971, Corollaire I.5.7.13] to the proper morphism $\bar{f}$ of algebraic spaces, we obtain a projective birational morphism $\bar{g}: \bar{X}^{\prime} \rightarrow \bar{X}$ such that $\bar{f} \bar{g}: \bar{X}^{\prime} \rightarrow \bar{Y}$ is projective. Let $g: X^{\prime} \rightarrow X$ be the base change of $\bar{g}$. Let $U$ be a dense open substack of $X$ such that $g$ induces an isomorphism $g^{-1}(U) \stackrel{\sim}{\rightarrow} U$. Let $j$ and $j^{\prime}$ be the open immersions, as shown in the commutative diagram

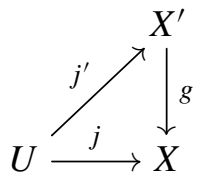

By [Beilinson et al. 1982, Corollaire 5.3.11] (see also Lemma 2.2.8), we have

$$
A \simeq j_{! *} j^{*} A \oplus B,
$$

where $B \in \operatorname{Perv}\left(X, \overline{\mathbb{Q}}_{\ell}\right)$ is supported on $X \backslash U$. By parts (4) and (8) of Remark 3.2.8, we have $j_{! *} j^{*} A \in \mathrm{D}_{\iota, \sigma}^{w}\left(X, \overline{\mathbb{Q}}_{\ell}\right)$. By the two-out-of-three property, $B \in \mathrm{D}_{\iota, \sigma}^{w}\left(X, \overline{\mathbb{Q}}_{\ell}\right)$. Since $g$ is projective, by Proposition 3.2.12 we have $\operatorname{Rg}_{*} j_{! *}^{\prime} j^{*} A \in \mathrm{D}_{\iota, \sigma}^{w}\left(X, \overline{\mathbb{Q}}_{\ell}\right)$, so

$$
\mathrm{R} g_{*} j_{! *}^{\prime} j^{*} A \simeq j_{! *} j^{*} A \oplus C,
$$

where $C \in \mathrm{D}_{\iota, \sigma}^{w}\left(X, \overline{\mathbb{Q}}_{\ell}\right)$ is supported on $X \backslash U$. Now by applying $\mathrm{R} f_{*}$ to $A \oplus C \simeq$ $\mathrm{R}_{*} j_{! *}^{\prime} j^{*} A \oplus B$, we obtain

$$
\mathrm{R} f_{*} A \oplus \mathrm{R} f_{*} C \simeq \mathrm{R} f_{*} \mathrm{R} g_{*} j_{! *}^{\prime} j^{*} A \oplus \mathrm{R} f_{*} B .
$$

By the induction hypothesis, $\mathrm{R} f_{*} B$ and $\mathrm{R} f_{*} C$ belong to $\mathrm{D}_{\iota, \sigma}^{w}\left(Y, \overline{\mathbb{Q}}_{\ell}\right)$. As $f g$ is projective, by Proposition 3.2.12 we have $\mathrm{R}(f g)_{*} j_{! *}^{\prime} j^{*} A \in \mathrm{D}_{\iota, \sigma}^{w}\left(Y, \overline{\mathbb{Q}}_{\ell}\right)$. It then follows from the two-out-of-three property that $\mathrm{R} f_{*} A$ belongs to $\mathrm{D}_{\iota, \sigma}^{w}\left(Y, \overline{\mathbb{Q}}_{\ell}\right)$.

Remark 3.2.13 (Gabber). In the case $Y=\operatorname{Spec}\left(\mathbb{F}_{q}\right)$, the proof of Theorem 3.2.3 still makes use of the relative hard Lefschetz theorem (applied to the morphism $g$ ). 
With the help of a refined Chow's lemma, it is possible to prove this case of Theorem 3.2.3 using only the absolute hard Lefschetz theorem, at least in the case of schemes.

The following is a preservation result for the exterior tensor power functor (-) $)^{\bigotimes m}$. Unlike the functors listed in Remark 3.2.8, (- $)^{\bigotimes m}$ is not additive and the reduction to the case of perverse sheaves is not trivial.

Proposition 3.2.14. Let $A$ be an l-mixed $\overline{\mathbb{Q}}_{\ell}$-complex of integral weights on $X$ such that, for all $n, w \in \mathbb{Z}, \operatorname{gr}_{w}^{W} p_{\mathscr{H}^{n}} A$ is $(-1)^{w} \sigma$-self-dual with respect to $K_{X}(-w)$. Then $\operatorname{gr}_{w}^{W} p_{H^{n}}\left(A^{\bigotimes m}\right)$ is $(-1)^{w} \sigma^{m}$-self-dual with respect to $K_{\left[X^{m} / \mathfrak{S}_{m}\right]}(-w)$ for all $n, w \in \mathbb{Z}$. Here $W$ denotes the $\iota$-weight filtrations. In particular, the functor $(-)^{\bigotimes m}$ carries $\mathrm{D}_{\iota, \sigma}^{w}\left(X, \overline{\mathbb{Q}}_{\ell}\right)$ to $\mathrm{D}_{\iota, \sigma^{m}}^{m w}\left(\left[X^{m} / \mathfrak{S}_{m}\right], \overline{\mathbb{Q}}_{\ell}\right)$.

Similar results hold for $\mathrm{D}_{\iota, \mathrm{d}}$.

Proof. Let $A^{n}=P^{p} \mathscr{H}^{n} A$. Then $\tau \in \mathfrak{S}_{m}$ acts on $X^{m}$ by $\left(x_{1}, \ldots, x_{m}\right) \mapsto\left(x_{\tau(1)}, \ldots, x_{\tau(m)}\right)$. By the Künneth formula,

$$
p_{\mathscr{H}^{n}}\left(A^{\bigotimes m}\right) \simeq \bigoplus_{n_{1}+\cdots+n_{m}=n} A^{n_{1}} \otimes \cdots \otimes A^{n_{m}},
$$

where $\tau$ acts on the right-hand side by $\prod_{i<j, \tau(i)>\tau(j)}(-1)^{n_{i} n_{j}}$ times the canonical isomorphism

$$
\tau^{*}\left(A^{n_{\tau(1)}} \otimes \cdots \otimes A^{n_{\tau(m)}}\right) \stackrel{\sim}{\longrightarrow} A^{n_{1}} \otimes \cdots \otimes A^{n_{m}} .
$$

Note that $W_{w} \mathscr{H}^{n}\left(A^{\bowtie m}\right) \subseteq p_{\mathscr{H}^{n}}\left(A^{\bigotimes m}\right)$ is the perverse subsheaf given by

$$
\sum_{\substack{n_{1}+\cdots+n_{m}=n \\ w_{1}+\cdots+w_{m}=w}} W_{w_{1}} A^{n_{1}} \otimes \cdots \otimes W_{w_{m}} A^{n_{m}} .
$$

So

$$
\operatorname{gr}_{w}^{W} p_{\mathscr{H}}^{n}\left(A^{\bigotimes m}\right) \simeq \bigoplus_{\substack{n_{1}+\cdots+n_{m}=n \\ w_{1}+\cdots+w_{m}=w}} \operatorname{gr}_{w_{1}}^{W} A^{n_{1}} \otimes \cdots \otimes \operatorname{gr}_{w_{1}}^{W} A^{n_{m}} .
$$

Thus the $(-1)^{w_{i}} \sigma$-symmetric isomorphisms

$$
\operatorname{gr}_{w_{i}}^{W} A^{n_{i}} \stackrel{\sim}{\longrightarrow}\left(D_{X} \operatorname{gr}_{w_{i}}^{W} A^{n_{i}}\right)\left(-w_{i}\right)
$$

induce a $(-1)^{w} \sigma^{m}$-symmetric isomorphism

$$
\operatorname{gr}_{w}^{W} p_{\mathscr{H}^{n}}\left(A^{\llbracket m}\right) \stackrel{\sim}{\longrightarrow}\left(D_{X^{m}} \operatorname{gr}_{w}^{W} p_{\mathscr{H}^{n}}\left(A^{\bigotimes m}\right)\right)(-w),
$$

compatible with the actions of $\mathfrak{S}_{m}$.

We conclude this subsection with a symmetry criterion in terms of traces of squares of Frobenius, an analogue of the Frobenius-Schur indicator theorem in representation theory (see [Serre 1998, Section 13.2, Proposition 39; Bröcker and tom 
Dieck 1995, Proposition II.6.8]). This criterion will be used to show a result on the independence of $\ell$ of symmetry (Corollary 4.2.14). We refer the reader to [Katz 2005, Theorem 1.9.6] for a related criterion on the symmetry of the geometric monodromy. For a groupoid $\mathscr{C}$, we let $|\mathscr{C}|$ denote the set of isomorphism classes of its objects.

Proposition 3.2.15. Let $X$ be a Deligne-Mumford stack of finite presentation over $\mathbb{F}_{q}$, connected and geometrically unibranch of dimension $d$. Let $\mathscr{F}$ be a semisimple lisse $\overline{\mathbb{Q}}_{\ell}$-sheaf on $X$, punctually $\iota$-pure of weight $w \in \mathbb{Z}$. Consider the series

$$
L^{(2)}(T)=\exp \left(\sum_{m \geq 1} \sum_{x \in\left|X\left(\mathbb{F}_{q^{m}}\right)\right|} \frac{\iota \operatorname{tr}\left(\operatorname{Frob}_{x}^{2} \mid \mathscr{F}_{\bar{x}}\right)}{\# \operatorname{Aut}(x)} \frac{T^{m}}{m}\right),
$$

where $\bar{x}$ denotes a geometric point above $x$, and where Frob $_{x}=$ Frob $_{q^{m}}$. Then the series $L^{(2)}(T)$ converges absolutely for $|T|<q^{-w-d}$ and extends to a rational function satisfying

$-\operatorname{ord}_{T=q^{-w-d}} L^{(2)}(T)$

$$
=\operatorname{dim} \mathrm{H}^{0}\left(X,\left(\operatorname{Sym}^{2}\left(\mathscr{F}^{\vee}\right)\right)(-w)\right)-\operatorname{dim} \mathrm{H}^{0}\left(X,\left(\wedge^{2}\left(\mathscr{F}^{\vee}\right)\right)(-w)\right) .
$$

In particular, if $\mathscr{F}$ is simple and $\sigma$-self-dual (resp. not self-dual) with respect to $\overline{\mathbb{Q}}_{\ell}(-w)$, then

$$
-\operatorname{ord}_{T=q^{-w-d}} L^{(2)}(T)=\sigma \quad(\text { resp. }=0) .
$$

Proof. For $x \in X\left(\mathbb{F}_{q^{m}}\right)$,

$$
\operatorname{tr}\left(\operatorname{Frob}_{x}^{2} \mid \mathscr{F}_{\bar{x}}\right)=\operatorname{tr}\left(\operatorname{Frob}_{x} \mid \operatorname{Sym}^{2} \mathscr{F}_{\bar{x}}\right)-\operatorname{tr}\left(\operatorname{Frob}_{x} \mid \wedge^{2} \mathscr{F}_{\bar{x}}\right) .
$$

Thus $L^{(2)}(T)=L_{\iota}\left(X, \operatorname{Sym}^{2} \mathscr{F}, T\right) / L_{\iota}\left(X, \wedge^{2} \mathscr{F}, T\right)$ (see [Sun 2012b, Definition 4.1] for the definition of the $L$-series $\left.L_{\iota}(X,-, T)\right)$. Note that $\mathscr{F} \otimes \mathscr{F}$ is lisse punctually $\iota$-pure of weight $2 w$, and semisimple by a theorem of Chevalley [1955, Chapitre IV, Proposition 5.2]. The same holds for $\operatorname{Sym}^{2} \mathscr{F}$ and $\wedge^{2} \mathscr{F}$. For $\mathscr{G}$ lisse punctually $\iota$-pure of weight $2 w$ on $X$, the series $L_{\iota}(X, \mathscr{G}, T)$ converges absolutely for $|T|<q^{-w-d}$ and extends to a rational function

$$
\iota \prod_{i} \operatorname{det}\left(1-T \operatorname{Frob}_{q} \mid \mathrm{H}_{c}^{i}\left(X_{\overline{\mathbb{F}}_{q}}, \mathscr{G}\right)\right)^{(-1)^{i+1}}
$$

by [Sun 2012b, Theorem 4.2]. Only the factor $i=2 d$ may contribute to poles on the circle $|T|=q^{-w-d}$, by [Sun 2012b, Theorem 1.4]. For every dense open substack $U$ of $X$ such that $U_{\text {red }}$ is regular, we have

$$
\mathrm{H}_{c}^{2 d}\left(X_{\overline{\mathbb{F}}_{q}}, \mathscr{G}\right) \simeq \mathrm{H}^{0}\left(U_{\overline{\mathbb{F}}_{q}}, \mathscr{G}^{\vee}\right)^{\vee}(-d) \simeq \mathrm{H}^{0}\left(X_{\overline{\mathbb{F}}_{q}}, \mathscr{G}^{\vee}\right)^{\vee}(-d)
$$


Here in the second assertion we used the hypothesis on $X$, which implies that the homomorphism $\pi_{1}(U) \rightarrow \pi_{1}(X)$ is surjective. Therefore,

$$
-\operatorname{ord}_{T=q^{-w-d}} L_{\iota}(X, \mathscr{G}, T)=\operatorname{dim} \mathrm{H}^{0}\left(X, \mathscr{G}^{\vee}(-w)\right)
$$

for $\mathscr{G}$ semisimple.

3.3. Variant: semisimple complexes over a separably closed field. In this subsection, let $k$ be a separably closed field. We establish variants over $k$ of results of Section 3.2. The main result is a preservation result under proper direct image (Theorem 3.3.7). We also include an example of Gabber (Remark 3.3.13) showcasing the difference in parity of Betti numbers between zero and positive characteristics.

One key point in Section 3.2 is the relative hard Lefschetz theorem for pure perverse sheaves. If $k$ has characteristic zero, a conjecture of Kashiwara states that all semisimple perverse sheaves satisfy relative hard Lefschetz. Kashiwara's conjecture was proved by Drinfeld [2001] assuming de Jong's conjecture for infinitely many primes $\ell$, which was later proved by Gaitsgory [2007] for $\ell>2$.

Drinfeld's proof uses the techniques of reduction from $k$ to finite fields in [Beilinson et al. 1982, Section 6]. The reduction holds, in fact, without restriction on the characteristic of $k$ and provides a class of semisimple perverse sheaves over $k$ for which the relative hard Lefschetz theorem holds. Let us briefly recall the reduction. Let $X$ be a Deligne-Mumford stack of finite presentation over $k$. There exist a subring $R \subseteq k$ of finite type over $\mathbb{Z}[1 / \ell]$ and a Deligne-Mumford stack $X_{R}$ of finite presentation over $\operatorname{Spec}(R)$ such that $X \simeq X_{R} \otimes_{R} k$. For extra data $(\mathscr{T}, \mathscr{L})$ on $X$, we have an equivalence [Beilinson et al. 1982, 6.1.10]

$$
\mathrm{D}_{\mathscr{T}, \mathscr{L}}^{b}\left(X, \overline{\mathbb{Q}}_{\ell}\right) \stackrel{\sim}{\rightarrow} \mathrm{D}_{\mathscr{T}, \mathscr{L}}^{b}\left(X_{s}, \overline{\mathbb{Q}}_{\ell}\right)
$$

for every geometric point $s$ above a closed point of $\operatorname{Spec}(R)$, provided that $R$ is big enough (relative to the data $(\mathscr{T}, \mathscr{L})$ ). Here $X_{s}$ denotes the base change of $X_{R}$ by $s \rightarrow \operatorname{Spec}(R)$. Note that $s$ is the spectrum of an algebraic closure of a finite field. Each $A \in \mathrm{D}_{c}^{b}\left(X, \overline{\mathbb{Q}}_{\ell}\right)$ is contained in $\mathrm{D}_{\mathscr{T}, \mathscr{L}}^{b}\left(X, \overline{\mathbb{Q}}_{\ell}\right)$ for some $(\mathscr{T}, \mathscr{L})$.

Definition 3.3.1 (admissible semisimple complexes). Let $A$ be a semisimple perverse $\overline{\mathbb{Q}}_{\ell}$-sheaf on $X$. If $k$ is an algebraic closure of a finite field, we say that $A$ is admissible if there exists a Deligne-Mumford stack $X_{0}$ of finite presentation over a finite subfield $k_{0}$ of $k$, an isomorphism $X \simeq X_{0} \otimes_{k_{0}} k$, and a perverse $\overline{\mathbb{Q}}_{\ell}$-sheaf $A_{0}$ on $X_{0}$ such that $A$ is isomorphic to the pullback of $A_{0}$. More generally, if $k$ has characteristic $>0$, we say that $A$ is admissible if the images of $A$ under the equivalences (3-3-1), for all geometric points $s$ over a closed point of $\operatorname{Spec}(R)$, are admissible, for some $R$ big enough. If $k$ has characteristic zero, we adopt the convention that every semisimple perverse $\overline{\mathbb{Q}}_{\ell}$-sheaf is admissible. 
We say that a complex $B \in \mathrm{D}_{c}^{b}\left(X, \overline{\mathbb{Q}}_{\ell}\right)$ is admissible semisimple if we have $B \simeq \bigoplus_{i}\left(P_{\mathscr{H}^{i}} B\right)[-i]$ and if, for each $i$, the $i$-th perverse cohomology sheaf ${ }^{p} \mathscr{H}^{i} B$ is admissible semisimple.

Remark 3.3.2. In the case where $k$ is the algebraic closure of a finite field $k_{0}$, for $X_{0}$ as above, the pullback of an $\iota$-pure complex on $X_{0}$ to $X$ is admissible semisimple by the decomposition theorems [Beilinson et al. 1982, Théorèmes 5.3.8, 5.4.5] ([Sun 2012a] for the case of stacks). Conversely, if a semisimple perverse $\overline{\mathbb{Q}}_{\ell}$-sheaf $A$ on $X$ is the pullback of $A_{0}$ on $X_{0}$ as above, then we may take $A_{0}$ to be pure (of weight 0 , for example) by Lafforgue's theorem [2002, Corollaire VII.8], mentioned in Remark 3.1.6(2).

Remark 3.3.3. Following [Beilinson et al. 1982, 6.2.4], we say that a simple perverse $\overline{\mathbb{Q}}_{\ell}$-sheaf on $X$ is of geometric origin if it belongs to the class of simple perverse $\overline{\mathbb{Q}}_{\ell}$-sheaves generated from the constant sheaf $\overline{\mathbb{Q}}_{\ell}$ on $\operatorname{Spec}(k)$ by taking composition factors of perverse cohomology sheaves under the six operations. By [Beilinson et al. 1982, Lemme 6.2.6] (suitably extended), simple perverse $\overline{\mathbb{Q}}_{\ell}$-sheaves of geometric origin are admissible.

The operations that preserve purity also preserve admissible semisimple complexes. The details are given below.

Remark 3.3.4 (preservation of admissible semisimple complexes). Let $f: X \rightarrow Y$ be a morphism.

- The full subcategory of $\mathrm{D}_{c}^{b}$ consisting of objects $A$ such that the composition factors of $P_{\mathscr{H}^{i}} A$ are admissible for all $i$ is stable under the operations $\mathrm{R} f_{*}$, $\mathrm{R} f_{!}, f^{*}, \mathrm{R} f^{!}, \otimes, \mathrm{R} \mathscr{H} o m$, and $(-)^{\bigotimes m}$.

- $D_{X}: \mathrm{D}_{c}^{b}\left(X, \overline{\mathbb{Q}}_{\ell}\right)^{\mathrm{op}} \rightarrow \mathrm{D}_{c}^{b}\left(X, \overline{\mathbb{Q}}_{\ell}\right)$ preserves admissible semisimple complexes.

- If $f$ is an open immersion, $f_{! *}$ preserves admissible semisimple perverse sheaves.

- Assume that $f$ is a closed immersion and let $A \in \mathrm{D}_{c}^{b}\left(X, \overline{\mathbb{Q}}_{\ell}\right)$. Then $A$ is admissible semisimple if and only if $f_{*} A$ is admissible semisimple.

- If $f$ is smooth, $f^{*}$ preserves admissible semisimple complexes.

- If $X$ and $Y$ are regular, $f^{*}$ preserves admissible semisimple complexes in $\mathrm{D}_{\text {lisse }}^{b}$.

- The functors

$$
\begin{gathered}
-\otimes-: \mathrm{D}_{c}^{b}\left(X, \overline{\mathbb{Q}}_{\ell}\right) \times \mathrm{D}_{c}^{b}\left(X^{\prime}, \overline{\mathbb{Q}}_{\ell}\right) \rightarrow \mathrm{D}_{c}^{b}\left(X \times X^{\prime}, \overline{\mathbb{Q}}_{\ell}\right), \\
(-)^{\bigotimes m}: \mathrm{D}_{c}^{b}\left(X, \overline{\mathbb{Q}}_{\ell}\right) \rightarrow \mathrm{D}_{c}^{b}\left(\left[X^{m} / \mathfrak{S}_{m}\right], \overline{\mathbb{Q}}_{\ell}\right), \quad m \geq 0,
\end{gathered}
$$

preserve admissible semisimple complexes.

- If $f$ is a proper morphism, $\mathrm{R} f_{*}$ preserves admissible semisimple complexes. 
These properties reduce to the corresponding properties for pure complexes over a finite field (Remark 3.2.7). Since the equivalences (3-3-1) are compatible with these operations, this reduction is clear in positive characteristic. The reduction in characteristic zero is more involved. The case of $\mathrm{R} f_{*}$ is done in [Drinfeld 2001] and the other cases can be done similarly.

Definition 3.3.5 $\left(\mathrm{D}_{\sigma}\right)$. We let

$$
\mathrm{D}_{\sigma}\left(X, \overline{\mathbb{Q}}_{\ell}\right) \subseteq \mathrm{Ob}\left(\mathrm{D}_{c}^{b}\left(X, \overline{\mathbb{Q}}_{\ell}\right)\right) \quad\left(\text { resp. } \mathrm{D}_{\mathrm{sd}}\left(X, \overline{\mathbb{Q}}_{\ell}\right) \subseteq \mathrm{Ob}\left(\mathrm{D}_{c}^{b}\left(X, \overline{\mathbb{Q}}_{\ell}\right)\right)\right)
$$

be the subset consisting of admissible semisimple complexes $A$ such that ${ }^{p{ }^{i} A}$ is $(-1)^{i} \sigma$-self-dual (resp. self-dual) with respect to $K_{X}$, for all $i$. We denote by $\mathrm{D}_{\mathrm{d}}\left(X, \overline{\mathbb{Q}}_{\ell}\right) \subseteq \mathrm{Ob}\left(\mathrm{D}_{c}^{b}\left(X, \overline{\mathbb{Q}}_{\ell}\right) \times \mathrm{D}_{c}^{b}\left(X, \overline{\mathbb{Q}}_{\ell}\right)\right)$ the subset consisting of pairs $(A, B)$ such that both $A$ and $B$ are admissible semisimple, and such that ${ }^{p} \mathscr{H}^{i} A$ is isomorphic to $D_{X}{ }^{p} \mathscr{H}^{i} B$.

By definition, we have $\mathrm{D}_{\mathrm{sd}}\left(X, \overline{\mathbb{Q}}_{\ell}\right)=\Delta^{-1}\left(\mathrm{D}_{\mathrm{d}}\left(X, \overline{\mathbb{Q}}_{\ell}\right)\right)$, where $\Delta: \mathrm{D}_{c}^{b}\left(X, \overline{\mathbb{Q}}_{\ell}\right) \rightarrow$ $\mathrm{D}_{c}^{b}\left(X, \overline{\mathbb{Q}}_{\ell}\right) \times \mathrm{D}_{c}^{b}\left(X, \overline{\mathbb{Q}}_{\ell}\right)$ is the diagonal embedding.

Example 3.3.6. For $X=\operatorname{Spec}(k)$, every object $A$ of $\mathrm{D}_{c}^{b}\left(X, \overline{\mathbb{Q}}_{\ell}\right)$ is admissible semisimple and belongs to $\mathrm{D}_{\text {sd }}\left(X, \overline{\mathbb{Q}}_{\ell}\right)$. Let $d_{i}=\operatorname{dim} \mathscr{H}^{i}(A)$. Then:

- $A$ is 1 -self-dual with respect to $\overline{\mathbb{Q}}_{\ell}$ if and only if it is self-dual with respect to $\overline{\mathbb{Q}}_{\ell}$, namely if $d_{i}=d_{-i}$ for all $i$. (Recall that in general self-dual objects are not necessarily 1 -self-dual.)

- $A$ is -1 -self-dual with respect to $\overline{\mathbb{Q}}_{\ell}$ if and only if $d_{i}$ equals $d_{-i}$ for all $i$ and $d_{0}$ is even.

- $A \in \mathrm{D}_{1}\left(X, \overline{\mathbb{Q}}_{\ell}\right)$ if and only if $d_{i}$ is even for $i$ odd.

- $A \in \mathrm{D}_{-1}\left(X, \overline{\mathbb{Q}}_{\ell}\right)$ if and only if $d_{i}$ is even for $i$ even.

- For $A, B \in \mathrm{D}_{c}^{b}\left(X, \overline{\mathbb{Q}}_{\ell}\right)$, we have $(A, B) \in \mathrm{D}_{\mathrm{d}}\left(X, \overline{\mathbb{Q}}_{\ell}\right)$ if and only if $A \simeq B$.

The main result of this subsection is the following.

Theorem 3.3.7. Let $f: X \rightarrow Y$ be a proper morphism of Deligne-Mumford stacks of finite presentation over $k$, where $Y$ has finite inertia. Then $R f_{*}$ preserves $D_{\sigma}$ and $D_{d}$.

Corollary 3.3.8. Let $f: X \rightarrow Y$ be a proper morphism, where $Y$ has finite inertia. Then $\mathrm{R} f_{*}$ preserves $\mathrm{D}_{\mathrm{sd}}$.

The strategy for proving Theorem 3.3.7 is the same as for Theorem 3.2.3. Let us recall that the operations listed in Remark 3.3.4 that preserve admissible semisimple complexes also preserve $\sigma$-self-dual complexes (Remark 2.1.4). With the exception of proper direct image $\mathrm{R} f_{*}$, they also preserve perversity, hence they preserve $\mathrm{D}_{\sigma}$. The details are given below. 
Remark 3.3.9 (preservation of $\mathrm{D}_{\sigma}$, easy part). Let $f: X \rightarrow Y$ be a morphism, and let $n \in \mathbb{Z}$.

(1) If $A \in \mathrm{D}_{\sigma}\left(X, \overline{\mathbb{Q}}_{\ell}\right)$, then $A[n] \in \mathrm{D}_{(-1)^{n} \sigma}\left(X, \overline{\mathbb{Q}}_{\ell}\right)$.

(2) $D_{X}$ preserves $\mathrm{D}_{\sigma}\left(X, \overline{\mathbb{Q}}_{\ell}\right)$.

(3) Assume that $X$ is regular and let $\mathscr{F}$ be a lisse $\overline{\mathbb{Q}}_{\ell}$-sheaf on $X$, admissible semisimple. Then there exists a nondegenerate $\sigma$-symmetric pairing $\mathscr{F} \otimes \mathscr{F} \rightarrow \overline{\mathbb{Q}}_{\ell}$ if and only if $\mathscr{F}$ belongs to $\mathrm{D}_{\sigma}\left(X, \overline{\mathbb{Q}}_{\ell}\right)$.

(4) If $f$ is smooth, then $f^{*}$ preserves $\mathrm{D}_{\sigma}$.

(5) If $X$ and $Y$ are regular, then $f^{*}$ preserves $\mathrm{D}_{\sigma} \cap \mathrm{D}_{\text {lisse }}^{b}$.

(6) Assume that $f$ is a closed immersion and let $A \in \mathrm{D}_{c}^{b}\left(X, \overline{\mathbb{Q}}_{\ell}\right)$. Then we have $A \in \mathrm{D}_{\sigma}\left(X, \overline{\mathbb{Q}}_{\ell}\right)$ if and only if $f_{*} A \in \mathrm{D}_{\sigma}\left(Y, \overline{\mathbb{Q}}_{\ell}\right)$.

(7) The exterior tensor product functors induce functors

$$
\begin{gathered}
-\otimes-: \mathrm{D}_{\sigma}\left(X, \overline{\mathbb{Q}}_{\ell}\right) \times \mathrm{D}_{\sigma^{\prime}}\left(X^{\prime}, \overline{\mathbb{Q}}_{\ell}\right) \rightarrow \mathrm{D}_{\sigma \sigma^{\prime}}\left(X \times X^{\prime}, \overline{\mathbb{Q}}_{\ell}\right), \\
(-)^{\bigotimes m}: \mathrm{D}_{\sigma}\left(X, \overline{\mathbb{Q}}_{\ell}\right) \rightarrow \mathrm{D}_{\sigma^{m}}\left(\left[X^{m} / \mathfrak{S}_{m}\right], \overline{\mathbb{Q}}_{\ell}\right), \quad m \geq 0 .
\end{gathered}
$$

For $(-)^{\unrhd m}$, the reduction to perverse sheaves is nontrivial and is similar to Proposition 3.2.14.

Similar properties hold for $\mathrm{D}_{\mathrm{d}}$.

By Proposition 2.2.1, the two-out-of-three property holds for $\mathrm{D}_{\sigma}$ and $\mathrm{D}_{\mathrm{d}}$.

We state a relative hard Lefschetz theorem over an arbitrary field $F$ in which $\ell$ is invertible.

Proposition 3.3.10 (relative hard Lefschetz). Let $f: X \rightarrow Y$ be a projective morphism of Deligne-Mumford stacks of finite presentation over $F$. Let $\eta \in \mathrm{H}^{2}\left(X, \overline{\mathbb{Q}}_{\ell}(1)\right)$ be the first Chern class of an $f$-ample invertible sheaf on $X$. Let $A$ be a perverse $\overline{\mathbb{Q}}_{\ell}$-sheaf on $X$ whose pullback to $X \otimes_{F} \bar{F}$ is admissible semisimple. Then, for $i \geq 0$, the morphism

$$
p_{\mathscr{H}^{-i}}\left(\eta^{i} \otimes \mathrm{id}_{A}\right):{ }^{p} \mathrm{R}^{-i} f_{*} A \rightarrow{ }^{p} \mathrm{R}^{i} f_{*} A(i)
$$

is an isomorphism.

That the morphism is an isomorphism can be checked on $X \otimes_{F} \bar{F}$. Thus we are reduced to the case where $F=k$ is separably closed. As mentioned earlier, the relative hard Lefschetz theorem in this case is obtained by reduction to the finite field case (Proposition 3.2.11).

Combining Proposition 3.3.10 with Lemma A.5.11, we obtain the following preservation result under projective direct image.

Proposition 3.3.11. Let $f: X \rightarrow Y$ be a projective morphism (over $k$ ). Then $\mathrm{R} f_{*}$ preserves $\mathrm{D}_{\sigma}$ and $\mathrm{D}_{\mathrm{d}}$. 
The proof of Theorem 3.2.3 can now be repeated verbatim to prove Theorem 3.3.7.

Over an arbitrary field $F$ in which $\ell$ is invertible, we may exploit the relative hard Lefschetz theorem to get analogues for split complexes that are geometrically semisimple.

Proposition 3.3.12. Let $f: X \rightarrow Y$ be a proper morphism of separated DeligneMumford stacks of finite type over $F$. Assume that $X$ is regular. Let $\mathscr{F}$ be a lisse $\overline{\mathbb{Q}}_{\ell}$-sheaf on $X$, whose pullback to $X_{\bar{F}}$ is admissible semisimple. Then we have $\mathrm{R} f_{*} \mathscr{F} \simeq \bigoplus_{i}\left({ }^{p} \mathrm{R}^{i} f_{*} \mathscr{F}\right)[-i]$.

Proof. By [Laumon and Moret-Bailly 2000, Théorème 16.6], there exists a finite surjective morphism $g_{1}: X^{\prime} \rightarrow X$ where $X^{\prime}$ is a scheme. Up to replacing $X^{\prime}$ by its normalization, we may assume that $X^{\prime}$ is normal. By de Jong's alterations [1996, Theorem 4.1], there exists a proper surjective morphism $g_{2}: X^{\prime \prime} \rightarrow X^{\prime}$, generically finite, such that $X^{\prime \prime}$ is regular and quasiprojective over $k$. Let $g=g_{1} g_{2}: X^{\prime \prime} \rightarrow X$. By the relative hard Lefschetz theorem (Proposition 3.3.10) and Deligne's decomposition theorem [1994], we have

$$
\mathrm{R}(f g)_{*} g^{*} \mathscr{F} \simeq \bigoplus_{i}\left({ }^{p} \mathrm{R}^{i}(f g)_{*} g^{*} \mathscr{F}\right)[-i] .
$$

Note that $g^{*} \mathscr{F} \simeq g^{*} \mathscr{F} \otimes \mathrm{R} g ! \overline{\mathbb{Q}}_{\ell} \simeq \mathrm{R} g ! \mathscr{F}$. Consider the composite

$$
\alpha: \mathscr{F} \rightarrow \mathrm{R} g_{*} g^{*} \mathscr{F} \simeq \mathrm{R} g_{!} \mathrm{R} g^{!} \mathscr{F} \rightarrow \mathscr{F}
$$

of the adjunction morphisms. Since $\alpha$ is generically multiplication by the degree of $g$, it is an isomorphism. It follows that $\mathscr{F}$ is a direct summand of $\mathrm{R} g_{*} g^{*} \mathscr{F}$, so that $\mathrm{R} f_{*} \mathscr{F}$ is a direct summand of $\mathrm{R}(f g)_{*} g^{*} \mathscr{F}$.

Remark 3.3.13 (Gabber). Let $X$ be a proper smooth algebraic space over $k$ and let $\mathscr{F}$ be a lisse $\overline{\mathbb{Q}}_{\ell}$-sheaf on $X$ with finite monodromy, -1 -self-dual with respect to $\overline{\mathbb{Q}}_{\ell}$. Then $\mathscr{F}$ is admissible semisimple (since each simple factor is of geometric origin), and $\mathscr{F}$ belongs to $\mathrm{D}_{-1}$. By Theorem 3.3.7, $b_{n}(\mathscr{F}):=\operatorname{dim} \mathrm{H}^{n}(X, \mathscr{F})$ is even for $n$ even.

If $k$ has characteristic 0 , then $b_{n}(\mathscr{F})$ is even for all $n$. To see this, we may assume $k=\mathbb{C}, X$ connected, and $\mathscr{F}$ simple. Let $G$ be the monodromy group of $\mathscr{F}$ and let $f: Y \rightarrow X$ be the corresponding Galois étale cover. Then

$$
\mathrm{H}^{n}(X, \mathscr{F}) \simeq \mathrm{H}^{n}\left(Y, f^{*} \mathscr{F}\right)^{G} \simeq\left(\mathrm{H}^{n}\left(Y, \overline{\mathbb{Q}}_{\ell}\right) \otimes_{\overline{\mathbb{Q}}_{\ell}} V\right)^{G},
$$

where $V$ is the representation of $G$ corresponding to $\mathscr{F}$. Thus $b_{n}(\mathscr{F})$ is the multiplicity of $V^{\vee}$ in the representation $\mathrm{H}^{n}\left(Y, \overline{\mathbb{Q}}_{\ell}\right)$ of $G$. Since the complex representation $\mathrm{H}^{n}(Y(\mathbb{C}), \mathbb{C})$ of $G$ has a real structure $\mathrm{H}^{n}(Y(\mathbb{C}), \mathbb{R})$, it admits a $G$-invariant nondegenerate symmetric bilinear form. In other words, it is 1-self-dual. The same holds for $\mathrm{H}^{n}\left(Y, \overline{\mathbb{Q}}_{\ell}\right)$. Therefore, the multiplicity of $V^{\vee}$, which is -1-self-dual, is 
necessarily even (see [Serre 1998, Section 13.2, Theorem 31; Bröcker and tom Dieck 1995, Proposition II.6.6 (i)-(iii)]).

By contrast, if $k$ has characteristic 2 or 3 , then $b_{n}(\mathscr{F})$ may be odd for $n$ odd, as shown by the following counterexample. Let $E$ be a supersingular elliptic curve over $k$ and let $G$ be its automorphism group. Let $X^{\prime} \rightarrow X$ be a finite étale cover of connected projective smooth curves over $k$ of Galois group $G$, which exists by [Pacheco and Stevenson 2000, Theorem 7.4], as explained in [Partsch 2013, Section 3]. Let $f: Y=\left(X^{\prime} \times E\right) / G \rightarrow X$ be the projection, where $G$ acts diagonally on $X^{\prime} \times E$. Then $\mathscr{F}=\mathrm{R}^{1} f_{*} \overline{\mathbb{Q}}_{\ell}$ is a -1 -self-dual simple lisse sheaf of rank 2 on $X$, of monodromy $G$. Note that $f^{*} \mathscr{F}$ is a -1 -self-dual simple lisse sheaf on $Y$ of monodromy $G$, since $\pi_{1}(Y)$ maps onto $\pi_{1}(X)$. We claim that $b_{1}\left(f^{*} \mathscr{F}\right)$ and $b_{1}(\mathscr{F})$ are not of the same parity. Indeed, consider the Leray spectral sequence for $\left(f, f^{*} \mathscr{F}\right)$ :

$$
E_{2}^{p q}=\mathrm{H}^{p}\left(X, R^{q} f_{*} f^{*} \mathscr{F}\right) \Rightarrow \mathrm{H}^{p+q}\left(Y, f^{*} \mathscr{F}\right) .
$$

By the projection formula,

$$
f_{*} f^{*} \mathscr{F} \simeq f_{*} \overline{\mathbb{Q}}_{\ell} \otimes \mathscr{F} \simeq \mathscr{F}, \quad \mathrm{R}^{1} f_{*} f^{*} \mathscr{F} \simeq \mathrm{R}^{1} f_{*} \overline{\mathbb{Q}}_{\ell} \otimes \mathscr{F}=\mathscr{F} \otimes \mathscr{F},
$$

so we have an exact sequence

$$
0 \rightarrow \mathrm{H}^{1}(X, \mathscr{F}) \rightarrow \mathrm{H}^{1}\left(Y, f^{*} \mathscr{F}\right) \rightarrow \mathrm{H}^{0}(X, \mathscr{F} \otimes \mathscr{F}) \rightarrow E_{2}^{20}=0 .
$$

Since $\operatorname{dim} \mathrm{H}^{0}(X, \mathscr{F} \otimes \mathscr{F})=1$, we get $b_{1}\left(f^{*} \mathscr{F}\right)=b_{1}(\mathscr{F})+1$. (That the Leray spectral sequence degenerates at $E_{2}$ also follows from a general theorem of Deligne [1968].) By the Grothendieck-Ogg-Shafarevich formula, $b_{1}(\mathscr{F})=(2 g-2) \operatorname{rk}(\mathscr{F})=4 g-4$ is even, where $g$ is the genus of $X$. It follows that $b_{1}\left(f^{*} \mathscr{F}\right)=4 g-3$ is odd.

\section{Symmetry in Grothendieck groups}

In Section 3, we studied the behavior of $\sigma$-self-dual pure perverse sheaves under operations that preserve purity. Mixed Hodge theory suggests that one may expect results for more general operations in the mixed case. This section confirms such expectations in a weak sense, by working in Grothendieck groups. We work over a finite field $k=\mathbb{F}_{q}$. In Section 4.1, we review operations on Grothendieck groups. In Section 4.2, we define certain subgroups of the Grothendieck groups and state the main result of this section (Theorem 4.2.5), which says that these subgroups are preserved by Grothendieck's six operations, and which contains the finite field case of Theorem 1.9. The proof is a bit involved and is given in Section 4.3.

4.1. Operations on Grothendieck groups. In this subsection, we review Grothendieck groups and operations on them. The six operations are easily defined. The 
action of the middle extension functor $f_{! *}$ on Grothendieck groups is more subtle, and we justify our definition with the help of purity.

Construction 4.1.1 (six operations on Grothendieck groups). Let $X$ be a DeligneMumford stack of finite presentation over a field. We let $\mathrm{K}\left(X, \overline{\mathbb{Q}}_{\ell}\right)$ denote the Grothendieck group of $\mathrm{D}_{c}^{b}\left(X, \overline{\mathbb{Q}}_{\ell}\right)$, which is a free abelian group generated by the isomorphism classes of simple perverse $\overline{\mathbb{Q}}_{\ell}$-sheaves. For an object $A$ of $\mathrm{D}_{c}^{b}\left(X, \overline{\mathbb{Q}}_{\ell}\right)$, we let $[A]$ denote its class in $\mathrm{K}\left(X, \overline{\mathbb{Q}}_{\ell}\right)$. The usual operations on derived categories induce maps between Grothendieck groups. More precisely, for a morphism $f: X \rightarrow Y$ of Deligne-Mumford stacks of finite presentation over a field, we have $\mathbb{Z}$-(bi)linear maps

$$
\begin{aligned}
&-\otimes-: \mathrm{K}\left(X, \overline{\mathbb{Q}}_{\ell}\right) \times \mathrm{K}\left(Y, \overline{\mathbb{Q}}_{\ell}\right) \rightarrow \mathrm{K}\left(X \times Y, \overline{\mathbb{Q}}_{\ell}\right), \\
&-\otimes-, \mathscr{H o m}(-,-): \mathrm{K}\left(X, \overline{\mathbb{Q}}_{\ell}\right) \times \mathrm{K}\left(X, \overline{\mathbb{Q}}_{\ell}\right) \rightarrow \mathrm{K}\left(X, \overline{\mathbb{Q}}_{\ell}\right), \\
& D_{X}: \mathrm{K}\left(X, \overline{\mathbb{Q}}_{\ell}\right) \rightarrow \mathrm{K}\left(X, \overline{\mathbb{Q}}_{\ell}\right), \\
& f^{*}, f^{!}: \mathrm{K}\left(Y, \overline{\mathbb{Q}}_{\ell}\right) \rightarrow \mathrm{K}\left(X, \overline{\mathbb{Q}}_{\ell}\right), \\
& f_{*}, f_{!}: \mathrm{K}\left(X, \overline{\mathbb{Q}}_{\ell}\right) \rightarrow \mathrm{K}\left(Y, \overline{\mathbb{Q}}_{\ell}\right) .
\end{aligned}
$$

The tensor product $(-\otimes-)$ endows $\mathrm{K}\left(X, \overline{\mathbb{Q}}_{\ell}\right)$ with a ring structure. The map $f^{*}$ is a ring homomorphism.

The Grothendieck ring is equipped with the structure of a $\lambda$-ring as follows. Readers not interested in this structure may skip this part as it is not used in the proof of the theorems in the Introduction. For $m \geq 0$, we have a map

$$
(-)^{\unrhd m}: \mathrm{K}\left(X, \overline{\mathbb{Q}}_{\ell}\right) \rightarrow \mathrm{K}\left(\left[X^{m} / \mathfrak{S}_{m}\right], \overline{\mathbb{Q}}_{\ell}\right),
$$

which preserves multiplication and satisfies $(n[A])^{\bigotimes m}=n^{m}[A]^{\bigotimes m}$ (with the convention $0^{0}=1$ ) for $n \geq 0$ and $(-[A])^{\bowtie m}=(-1)^{m}[\mathscr{S}] \otimes[A]^{\bowtie m}$, where $\mathscr{S}$ is the lisse sheaf of rank 1 on $\left[X^{m} / \mathfrak{S}_{m}\right]$ given by the sign character $\mathfrak{S}_{m} \rightarrow \overline{\mathbb{Q}}_{\ell}^{\times}$. The maps $\lambda^{m}: \mathrm{K}\left(X, \overline{\mathbb{Q}}_{\ell}\right) \rightarrow \mathrm{K}\left(X, \overline{\mathbb{Q}}_{\ell}\right)$ given by $\lambda^{m}(x)=(-1)^{m} p_{*} \Delta^{*}(-x)^{\bigotimes m}$, where $\Delta:$ $X \times B \mathfrak{S}_{m} \rightarrow\left[X^{m} / \mathfrak{S}_{m}\right]$ is the diagonal morphism and $p: X \times B \mathfrak{S}_{m} \rightarrow X$ is the projection, endow $\mathrm{K}\left(X, \overline{\mathbb{Q}}_{\ell}\right)$ with the structure of a special $\lambda$-ring. The map $f^{*}$ is a $\lambda$-ring homomorphism. We refer the reader to [Grothendieck 1958, Section 4] and [Atiyah and Tall 1969] for the definitions of special $\lambda$-ring and $\lambda$-ring homomorphism.

Remark 4.1.2. For a separated quasifinite morphism $f: X \rightarrow Y$, the functor $f_{!_{*}}: \operatorname{Perv}\left(X, \overline{\mathbb{Q}}_{\ell}\right) \rightarrow \operatorname{Perv}\left(Y, \overline{\mathbb{Q}}_{\ell}\right)$ is not exact in general. There exists a unique homomorphism $f_{! *}: \mathrm{K}\left(X, \overline{\mathbb{Q}}_{\ell}\right) \rightarrow \mathrm{K}\left(Y, \overline{\mathbb{Q}}_{\ell}\right)$ such that $f_{! *}[A]=\left[f_{! *} A\right]$ for $A$ perverse semisimple. As we shall see in Lemma 4.1.8, over a finite field this identity also holds for $A$ pure perverse.

We note the following consequence of Lemma 2.2.11 (applied to semisimple perverse sheaves). 
Lemma 4.1.3. Let $\left(X_{\alpha}\right)_{\alpha \in I}$ be a finite Zariski open covering of $X$. Let $A \in \mathrm{K}\left(X, \overline{\mathbb{Q}}_{\ell}\right)$. Then

$$
\sum_{\substack{J \subseteq I \\ \# J \text { even }}} j_{J ! *} j_{J}^{*} A=\sum_{\substack{J \subseteq I \\ \# J \text { odd }}} j_{J ! * j} j_{J}^{*} A
$$

where $j_{J}: \bigcap_{\alpha \in J} X_{\alpha} \rightarrow X$ is the open immersion.

In the rest of this section we work over a finite field $k=\mathbb{F}_{q}$. We first recall the following injectivity, which will be used in the proof of Corollary 4.2.10.

Lemma 4.1.4. The homomorphism $\mathrm{K}\left(X, \overline{\mathbb{Q}}_{\ell}\right) \rightarrow \operatorname{Map}\left(\coprod_{m \geq 1}\left|X\left(\mathbb{F}_{q^{m}}\right)\right|, \overline{\mathbb{Q}}_{\ell}\right)$ sending A to $x \mapsto \operatorname{tr}\left(\operatorname{Frob}_{x} \mid A_{\bar{x}}\right)$ is injective.

As in [Laumon 1987, Théorème 1.1.2], this injectivity follows from Chebotarev's density theorem [Serre 1965, Theorem 7], which extends to the case of DeligneMumford stacks as follows.

Lemma 4.1.5. Let $Y \rightarrow X$ be a Galois étale cover of irreducible Deligne-Mumford stacks of dimension d of finite presentation over $\mathbb{F}_{q}$, and let $G$ be the Galois group. Let $R \subseteq G$ be a subset stable under conjugation. Then

$$
\lim _{T \rightarrow\left(q^{-d}\right)^{-}} \sum_{m \geq 1} \sum_{x} \frac{1}{\# \operatorname{Aut}(x)} \frac{T^{m}}{m} / \log \frac{1}{T-q^{-d}}=\# R / \# G,
$$

where $x$ runs through isomorphism classes of $X\left(\mathbb{F}_{q^{m}}\right)$ such that the image $F_{x}$ of Frob $_{x}$ in $G$ (well-defined up to conjugation) lies in $R$.

Proof. For a character $\chi: G \rightarrow \overline{\mathbb{Q}}_{\ell}$ of a $\overline{\mathbb{Q}}_{\ell}$-representation of $G$, consider the $L$-series

$$
L(X, \iota \chi, T)=L_{\iota}\left(X, \mathscr{F}_{\chi}, T\right)=\exp \left(\sum_{m \geq 1} \sum_{x \in\left|X\left(\mathbb{F}_{q}\right)\right|} \frac{\iota \chi\left(F_{x}\right)}{\# \operatorname{Aut}(x)} \frac{T^{m}}{m}\right)
$$

associated to the corresponding lisse $\overline{\mathbb{Q}}_{\ell}$-sheaf $\mathscr{F}_{\chi}$ on $X$ [Sun 2012b, Definition 4.1]. The series $L(X, \iota \chi, T)$ converges absolutely for $|T|<q^{-d}$ and extends, by [Sun $2012 b$, Theorem 4.2], to a rational function

$$
\iota \prod_{i} \operatorname{det}\left(1-T \operatorname{Frob}_{q} \mid \mathrm{H}_{c}^{i}\left(X_{\overline{\mathbb{F}}_{q}}, \mathscr{F}_{\chi}\right)\right)^{(-1)^{i+1}} .
$$

As $\mathrm{H}_{c}^{2 d}\left(X_{\overline{\mathbb{F}}_{q}}, \mathscr{F}_{\chi}\right) \simeq \mathrm{H}^{0}\left(U_{\overline{\mathbb{F}}_{q}}, \mathscr{F}_{\chi}^{\vee}\right)^{\vee}(-d)$ for a dense open substack $U$ of $X$ such that $U_{\text {red }}$ is regular, $-\operatorname{ord}_{T=q^{-d}} L(X, \iota \chi, T)=\operatorname{dim}^{0}\left(U, \mathscr{F}_{\chi}^{\vee}\right)$ is the multiplicity of the identity character in $\chi$, so that

$$
\lim _{T \rightarrow\left(q^{-d}\right)^{-}} \sum_{m \geq 1} \sum_{x \in\left|X\left(\mathbb{F}_{q^{m}}\right)\right|} \frac{\iota \chi\left(F_{x}\right)}{\# \operatorname{Aut}(x)} \frac{T^{m}}{m} / \log \frac{1}{T-q^{-d}}=\sum_{g \in G} \iota \chi(g) / \# G .
$$


This equality extends to an arbitrary class function $\chi: G \rightarrow \overline{\mathbb{Q}}_{\ell}$. It then suffices to take $\chi$ to be the characteristic function of $R$.

Next we discuss purity.

Notation 4.1.6. For $w \in \mathbb{R}$, we let $\mathrm{K}_{l}^{w}\left(X, \overline{\mathbb{Q}}_{\ell}\right) \subseteq \mathrm{K}\left(X, \overline{\mathbb{Q}}_{\ell}\right)$ denote the subgroup generated by perverse sheaves $\iota$-pure of weight $w$ on $X$. We set $\mathrm{K}_{l}^{\mathbb{Z}}\left(X, \overline{\mathbb{Q}}_{\ell}\right):=$ $\bigoplus_{w \in \mathbb{Z}} \mathbf{K}_{\iota}^{w}\left(X, \overline{\mathbb{Q}}_{\ell}\right)$.

The group $\mathrm{K}_{l}^{w}\left(X, \overline{\mathbb{Q}}_{\ell}\right)$ is a free abelian group generated by the isomorphism classes of simple perverse sheaves $\iota$-pure of weight $w$ on $X$. We also have $\bigoplus_{w \in \mathbb{R}} \mathrm{K}_{l}^{w}\left(X, \overline{\mathbb{Q}}_{\ell}\right) \subseteq \mathrm{K}\left(X, \overline{\mathbb{Q}}_{\ell}\right)$, and the $\lambda$-subring $\mathrm{K}_{l}^{\mathbb{Z}}\left(X, \overline{\mathbb{Q}}_{\ell}\right) \subseteq \mathrm{K}\left(X, \overline{\mathbb{Q}}_{\ell}\right)$ is stable under Grothendieck's six operations and duality. For $w \in \mathbb{Z}$, the group $\mathrm{K}^{w}\left(X, \overline{\mathbb{Q}}_{\ell}\right):=$ $\bigcap_{\iota} \mathrm{K}_{\iota}^{w}\left(X, \overline{\mathbb{Q}}_{\ell}\right)$ is a free abelian group generated by the isomorphism classes of perverse sheaves pure of weight $w$ on $X$.

Remark 4.1.7. In fact, we have $\bigoplus_{w \in \mathbb{R}} \mathrm{K}_{l}^{w}\left(X, \overline{\mathbb{Q}}_{\ell}\right)=\mathrm{K}\left(X, \overline{\mathbb{Q}}_{\ell}\right)$, as every $\overline{\mathbb{Q}}_{\ell}$-sheaf on $X$ is $\iota$-mixed by Lafforgue's theorem [2002, Corollaire VII.8], mentioned in Remark 3.1.6(2).

For a subset $I \subseteq \mathbb{R}$, we let $\operatorname{Perv}_{\iota}^{I}\left(X, \overline{\mathbb{Q}}_{\ell}\right) \subseteq \operatorname{Perv}\left(X, \overline{\mathbb{Q}}_{\ell}\right)$ denote the full subcategory of perverse sheaves $\iota$-mixed of weights contained in $I$. Lemmas 4.1.8 and 4.1 .9 below, which justify the definition of the map $f_{! *}$ in Remark 4.1.2, are taken from [Zheng 2005, Lemme 2.9, Corollaire 2.10].

Lemma 4.1.8. Let $f: X \rightarrow Y$ be a separated quasifinite morphism. For $w \in \mathbb{R}$, the functor

$$
f_{! *}: \operatorname{Perv}_{\iota}^{\{w, w+1\}}\left(X, \overline{\mathbb{Q}}_{\ell}\right) \rightarrow \operatorname{Perv}_{\iota}^{\{w, w+1\}}\left(Y, \overline{\mathbb{Q}}_{\ell}\right)
$$

is exact. In particular, $f_{!_{*}}[A]=\left[f_{! *} A\right]$ for $A \in \operatorname{Perv}_{\iota}^{\{w, w+1\}}\left(X, \overline{\mathbb{Q}}_{\ell}\right)$.

Proof. As the assertion is local for the étale topology on $Y$ and trivial for $f$ proper quasifinite, we may assume that $f$ is an open immersion. Let $i: Z \rightarrow Y$ be the closed immersion complementary to $f$. We proceed by induction on the dimension $d$ of $Z$. Let $0 \rightarrow A_{1} \rightarrow A_{2} \rightarrow A_{3} \rightarrow 0$ be a short exact sequence in $\operatorname{Perv}_{l}^{\{w, w+1\}}\left(X, \overline{\mathbb{Q}}_{\ell}\right)$. As in Gabber's proof of his theorem on the independence on $\ell$ for middle extensions [Fujiwara 2002, Theorem 3], up to shrinking $Z$, we may assume that $Z$ is smooth equidimensional and that $\mathscr{H}^{n} i^{*} \mathrm{R} f_{*} A_{j}$ is lisse for every $j$ and every $n$. It follows that the distinguished triangle

$$
i_{*} \mathrm{R} i^{!} f_{! *} A_{j} \rightarrow f_{! *} A_{j} \rightarrow \mathrm{R} f_{*} A_{j} \rightarrow
$$

induces isomorphisms $f_{! *} A_{j} \stackrel{\sim}{\longrightarrow}{ }^{P} \tau=-d-1 \mathrm{R} f_{*} A_{j}$ and ${ }^{P_{\tau} \geq-d} \mathrm{R} f_{*} A_{j} \stackrel{\sim}{\rightarrow} i_{*} \mathrm{R} i ! f_{! *} A_{j}[1]$ for every $j$. Here $P$ denotes the $t$-structure obtained by gluing $\left(\mathrm{D}_{c}^{b}\left(X, \overline{\mathbb{Q}}_{\ell}\right), 0\right)$ and the canonical $t$-structure on $\mathrm{D}_{c}^{b}\left(Z, \overline{\mathbb{Q}}_{\ell}\right)$. Thus ${ }^{P} \mathrm{R}^{-d-1} f_{*} A_{j} \simeq i_{*} \mathscr{H}^{-d-1} i^{*} f_{! *} A_{j}$ has punctual $\iota$-weights $\leq w-d$, while ${ }^{P} \mathrm{R}^{-d} f_{*} A_{j} \simeq i_{*} \mathscr{H}^{-d+1} \mathrm{R}^{!} f_{! *} A_{j}$ has punctual 
l-weights $\geq w-d+1$. Therefore, the morphism ${ }^{P} \mathrm{R}^{-d-1} f_{*} A_{3} \rightarrow{ }^{P} \mathrm{R}^{-d} f_{*} A_{1}$ is zero. Applying Lemma 4.1.9 below, we get a distinguished triangle

$$
{ }^{P_{\tau} \leq-d-1} \mathrm{R} f_{*} A_{1} \rightarrow{ }^{P_{\tau} \leq-d-1} \mathrm{R} f_{*} A_{2} \rightarrow{ }^{P_{\tau} \leq-d-1} \mathrm{R} f_{*} A_{3} \rightarrow .
$$

Taking perverse cohomology sheaves, we get the exactness of the sequence

$$
0 \rightarrow f_{!_{*}} A_{1} \rightarrow f_{!_{*}} A_{2} \rightarrow f_{! *} A_{3} \rightarrow 0 .
$$

Lemma 4.1.9. Let $P$ be a $t$-structure on a triangulated category $\mathscr{D}$ and let $A \stackrel{a}{\longrightarrow}$ $B \stackrel{b}{\longrightarrow} C \stackrel{c}{\longrightarrow} A[1]$ be a distinguished triangle such that ${ }^{P} \mathrm{H}^{0} c:{ }^{P} \mathrm{H}^{0} C \rightarrow{ }^{P} \mathrm{H}^{1} A$ is zero. Then there exists a unique nine-diagram of the form

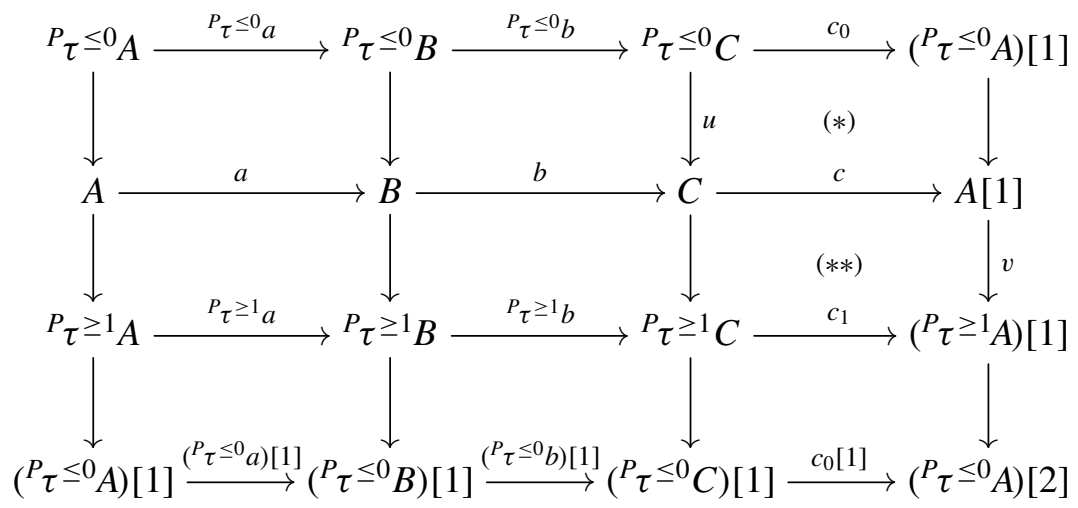

in which the columns are the canonical distinguished triangles.

By a nine-diagram in a triangulated category (see Proposition 1.1.11 of [Beilinson et al. 1982]), we mean a diagram

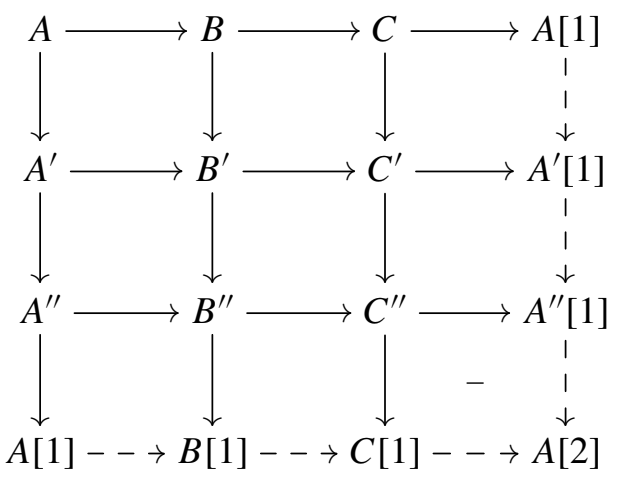

in which the square marked with "-" is anticommutative and all other squares are commutative, the dashed arrows are induced from the solid ones by translation, and the rows and columns in solid arrows are distinguished triangles. 
Proof. First note that $v c u$ is the image of ${ }^{P} \mathrm{H}^{0} c$ under the isomorphism

$$
\operatorname{Hom}\left({ }^{P} \mathrm{H}^{0} C,{ }^{P} \mathrm{H}^{1} A\right) \stackrel{\sim}{\longrightarrow} \operatorname{Hom}\left({ }^{P} \tau^{\leq 0} C,\left({ }^{P} \tau^{\geq 1} A\right)[1]\right) .
$$

Hence $v c u=0$. Moreover, $\operatorname{Hom}\left({ }^{P_{\tau} \leq 0} C,{ }^{P_{\tau} \geq 1} A\right)=0$. Thus by [Beilinson et al. 1982, Proposition 1.1.9], there exist a unique $c_{0}$ making $(*)$ commutative and a unique $c_{1}$ making $(* *)$ commutative. This proves the uniqueness of (4-1-1). It remains to show that (4-1-1) thus constructed is a nine-diagram. To do this, we extend the upper left square of (4-1-1) into a nine-diagram

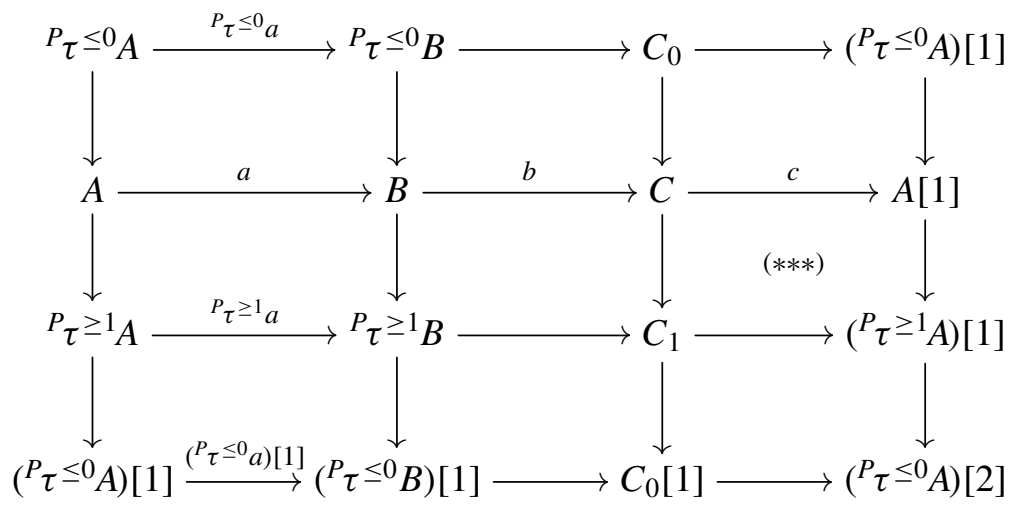

By the first and third rows of (4-1-2), $C_{0} \in{ }^{P} \mathscr{D} \leq 0$ and $C_{1} \in{ }^{P} \mathscr{D} \geq 0$. Taking ${ }^{P} \mathrm{H}^{0}$ of $(* * *)$, we obtain a commutative diagram

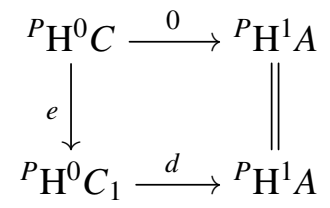

in which $e$ is an epimorphism and $d$ is a monomorphism. Thus ${ }^{P} \mathrm{H}^{0} C_{1}=0$, so that $C_{1} \in{ }^{P} \mathscr{D}^{\geq 1}$. Further applying [Beilinson et al. 1982, Proposition 1.1.9], we may identify (4-1-2) with (4-1-1).

4.2. Statement and consequences of main result. In this subsection, we define a subgroup $\mathrm{K}_{\iota, \sigma}$ of the Grothendieck group and state its preservation by Grothendieck's six operations, given in Theorem 4.2 .5 which contains the finite field case of Theorem 1.9. We then give a number of consequences and discuss the relationship with the independence of $\ell$ and Laumon's theorem on Euler characteristics.

Definition 4.2.1 $\left(\mathrm{K}_{\iota, \sigma}\right)$. We define $\mathrm{K}_{\iota, \sigma}^{w}\left(X, \overline{\mathbb{Q}}_{\ell}\right) \subseteq \mathrm{K}_{\iota}^{w}\left(X, \overline{\mathbb{Q}}_{\ell}\right)\left(\operatorname{resp} . \mathrm{K}_{\iota, \mathrm{sd}}^{w}\left(X, \overline{\mathbb{Q}}_{\ell}\right) \subseteq\right.$ $\left.\mathrm{K}_{\iota}^{w}\left(X, \overline{\mathbb{Q}}_{\ell}\right)\right)$, for $w \in \mathbb{Z}$, to be the subgroup generated by [B], for $B$ perverse, $\iota$-pure 
of weight $w$, and $(-1)^{w} \sigma$-self-dual (resp. self-dual) with respect to $K_{X}(-w)$. We set

$$
\mathrm{K}_{\iota, \sigma}\left(X, \overline{\mathbb{Q}}_{\ell}\right)=\bigoplus_{w \in \mathbb{Z}} \mathrm{K}_{\iota, \sigma}^{w}\left(X, \overline{\mathbb{Q}}_{\ell}\right) \quad\left(\operatorname{resp} . \mathrm{K}_{\iota, \mathrm{sd}}\left(X, \overline{\mathbb{Q}}_{\ell}\right)=\bigoplus_{w \in \mathbb{Z}} \mathrm{K}_{\iota, \mathrm{sd}}^{w}\left(X, \overline{\mathbb{Q}}_{\ell}\right)\right) .
$$

We define the twisted dualizing map

$$
\bar{D}_{\iota, X}: \mathrm{K}_{l}^{\mathbb{Z}}\left(X, \overline{\mathbb{Q}}_{\ell}\right) \rightarrow \mathrm{K}_{l}^{\mathbb{Z}}\left(X, \overline{\mathbb{Q}}_{\ell}\right)
$$

to be the direct sum of the group automorphisms $\bar{D}_{\iota, X}^{w}: \mathrm{K}_{\iota}^{w}\left(X, \overline{\mathbb{Q}}_{\ell}\right) \rightarrow \mathrm{K}_{\iota}^{w}\left(X, \overline{\mathbb{Q}}_{\ell}\right)$ sending $[A]$ to $\left[\left(D_{X} A\right)(-w)\right]$. We let $\mathrm{K}_{l, \mathrm{~d}}^{w}\left(X, \overline{\mathbb{Q}}_{\ell}\right) \subseteq \mathrm{K}_{l}^{w}\left(X, \overline{\mathbb{Q}}_{\ell}\right)^{2}$ denote the graph of $\bar{D}_{\iota, X}^{w}$. We set

$$
\mathrm{K}_{\iota, \mathrm{d}}\left(X, \overline{\mathbb{Q}}_{\ell}\right)=\bigoplus_{w \in \mathbb{Z}} \mathrm{K}_{\iota, \mathrm{d}}^{w}\left(X, \overline{\mathbb{Q}}_{\ell}\right)
$$

Note that $\bar{D}_{\iota, X} \bar{D}_{\iota, X}=\mathrm{id}$, and that $\mathrm{K}_{\iota, \mathrm{d}}\left(X, \overline{\mathbb{Q}}_{\ell}\right) \subseteq \mathrm{K}_{\iota}^{\mathbb{Z}}\left(X, \overline{\mathbb{Q}}_{\ell}\right)^{2}$ is the graph of $\bar{D}_{\iota, X}$. Example 4.2.2. For $X=\operatorname{Spec}\left(\mathbb{F}_{q}\right)$, an element $A \in \mathrm{K}\left(X, \overline{\mathbb{Q}}_{\ell}\right)$ is determined by the determinant

$$
P(A, T):=\operatorname{det}\left(1-T \operatorname{Frob}_{q} \mid A_{\overline{\mathbb{F}}_{q}}\right) \in \overline{\mathbb{Q}}_{\ell}(T) .
$$

Assume $A \in \mathrm{K}_{l}^{w}\left(X, \overline{\mathbb{Q}}_{\ell}\right), w \in \mathbb{Z}$. For $\lambda \in \overline{\mathbb{Q}}_{\ell}$ satisfying $|\iota(\lambda)|=q^{w / 2}$, we let $m_{\lambda}$ and $m_{\lambda}^{\prime}$ denote the order at $T=1 / \lambda$ of $P(A, T)$ and $P\left(\bar{D}_{\iota, X} A, T\right)$, respectively. We then have $m_{\lambda}=m_{q^{w} / \lambda}^{\prime}$; in other words,

$$
\iota P(A, T)=\bar{\imath} P\left(\bar{D}_{\iota, X} A, T\right) .
$$

We also have $\mathrm{K}_{l,(-1)^{w+1}}^{w}\left(X, \overline{\mathbb{Q}}_{\ell}\right) \subseteq \mathrm{K}_{\iota,(-1)^{w}}^{w}\left(X, \overline{\mathbb{Q}}_{\ell}\right)=\mathrm{K}_{\iota, \mathrm{sd}}^{w}\left(X, \overline{\mathbb{Q}}_{\ell}\right)$. The following conditions are equivalent:

(1) $A \in \mathrm{K}_{\iota,(-1)^{w}}^{w}\left(X, \overline{\mathbb{Q}}_{\ell}\right)=\mathrm{K}_{\iota, \mathrm{sd}}^{w}\left(X, \overline{\mathbb{Q}}_{\ell}\right)$;

(2) $m_{\lambda}=m_{q^{w} / \lambda}$ for all $\lambda$;

(3) $\iota P(A, T) \in \mathbb{R}(T)$.

Furthermore, the following conditions are equivalent:

(1) $A \in \mathrm{K}_{\iota,(-1)^{w+1}}^{w}\left(X, \overline{\mathbb{Q}}_{\ell}\right)$;

(2) $m_{q^{w / 2}}, m_{-q^{w / 2}}$ are even, and we have $m_{\lambda}=m_{q^{w} / \lambda}$ for all $\lambda$;

(3) the rank $b=\sum_{\lambda} m_{\lambda} \in \mathbb{Z}$ of $A$ is even, and we have $\iota P(A, T) \in \mathbb{R}(T)$ and $\operatorname{det}\left(\operatorname{Frob}_{q} \mid A_{\overline{\mathbb{F}}_{q}}\right)=q^{w b / 2}$.

Remark 4.2.3. Let $w \in \mathbb{Z}$.

(1) By definition, $\mathrm{K}_{\iota, \sigma}\left(X, \overline{\mathbb{Q}}_{\ell}\right) \subseteq \mathrm{K}\left(X, \overline{\mathbb{Q}}_{\ell}\right)\left(\right.$ resp. $\left.\mathrm{K}_{\iota, \text { sd }}\left(X, \overline{\mathbb{Q}}_{\ell}\right) \subseteq \mathrm{K}\left(X, \overline{\mathbb{Q}}_{\ell}\right)\right)$ is generated by the image of $\mathrm{D}_{\iota, \sigma}^{w}\left(X, \overline{\mathbb{Q}}_{\ell}\right)\left(\right.$ resp. $\left.\mathrm{D}_{\iota, \mathrm{sd}}^{w}\left(X, \overline{\mathbb{Q}}_{\ell}\right)\right)$, from Definition 3.2.2. Moreover, $\mathrm{K}_{\iota, \mathrm{d}}\left(X, \overline{\mathbb{Q}}_{\ell}\right) \subseteq \mathrm{K}\left(X, \overline{\mathbb{Q}}_{\ell}\right)^{2}$ is generated by the image of $\mathrm{D}_{\iota, \mathrm{d}}^{w}\left(X, \overline{\mathbb{Q}}_{\ell}\right)$. 
(2) By Remark 2.2.7, in the definition of $\mathrm{K}_{l, \sigma}^{w}$, one may restrict to semisimple perverse sheaves. This also holds for $\mathrm{K}_{\iota, \mathrm{sd}}^{w}$. Thus $\mathrm{K}_{l, \sigma}^{w}\left(X, \overline{\mathbb{Q}}_{\ell}\right)\left(\operatorname{resp} . \mathrm{K}_{\iota, \mathrm{sd}}^{w}\left(X, \overline{\mathbb{Q}}_{\ell}\right)\right)$ is generated by $[A]+\left[\left(D_{X} A\right)(-w)\right]$ for $A$ simple perverse $\iota$-pure of weight $w$, and $[B]$ for $B$ simple perverse $\iota$-pure of weight $w$ and $(-1)^{w} \sigma$-self-dual (resp. self-dual) with respect to $K_{X}(-w)$.

(3) By Proposition 2.2.3, we have $\mathrm{K}_{\iota, \mathrm{sd}}^{w}\left(X, \overline{\mathbb{Q}}_{\ell}\right)=\mathrm{K}_{\iota, 1}^{w}\left(X, \overline{\mathbb{Q}}_{\ell}\right)+\mathrm{K}_{\iota,-1}^{w}\left(X, \overline{\mathbb{Q}}_{\ell}\right)$.

(4) $\mathrm{K}_{\iota, \mathrm{d}}\left(X, \overline{\mathbb{Q}}_{\ell}\right)$ is generated by $\left([B],\left[\left(D_{X} A\right)(-w)\right]\right)$ for $B$ simple perverse $\iota$-pure of weight $w$. Thus $\mathrm{K}_{\iota, \mathrm{sd}}\left(X, \overline{\mathbb{Q}}_{\ell}\right)=\Delta^{-1}\left(\mathrm{~K}_{\iota, \mathrm{d}}\left(X, \overline{\mathbb{Q}}_{\ell}\right)\right)$, where $\Delta: \mathrm{K}_{l}^{\mathbb{Z}}\left(X, \overline{\mathbb{Q}}_{\ell}\right) \rightarrow$ $\mathrm{K}_{l}^{\mathbb{Z}}\left(X, \overline{\mathbb{Q}}_{\ell}\right)^{2}$ is the diagonal embedding. In other words, for $A \in \mathrm{K}_{l}^{\mathbb{Z}}\left(X, \overline{\mathbb{Q}}_{\ell}\right)$, $A$ belongs to $\mathrm{K}_{\iota, \mathrm{sd}}\left(X, \overline{\mathbb{Q}}_{\ell}\right)$ if and only if $A=\bar{D}_{\iota, X} A$.

(5) For $A \in \mathrm{K}_{l}^{\mathbb{Z}}\left(X, \overline{\mathbb{Q}}_{\ell}\right)$ and $n \in \mathbb{Z}$, we have $\bar{D}_{\iota, X}(A(n))=\left(\bar{D}_{\iota, X} A\right)(n)$. For $A \in \mathrm{K}_{l, \sigma}^{w}\left(X, \overline{\mathbb{Q}}_{\ell}\right)$, we have $A(n) \in \mathrm{K}_{l, \sigma}^{w-2 n}\left(X, \overline{\mathbb{Q}}_{\ell}\right)$.

(6) Let $A$ be a perverse sheaf on $X, \iota$-pure of weight $w$. By Corollary 2.2.6, $[A] \in \mathrm{K}_{l, \sigma}^{w}\left(X, \overline{\mathbb{Q}}_{\ell}\right)$ if and only if the semisimplification of $A$ is $(-1)^{w} \sigma$-selfdual with respect to $K_{X}(-w)$. Similar results hold for $\mathrm{K}_{\iota, \mathrm{sd}}$ and $\mathrm{K}_{\iota, \mathrm{d}}$.

Remark 4.2.4. Although we do not need it in the sequel, let us give two more descriptions of $\mathrm{K}_{\iota, \sigma}^{w}$. In our definition of $\mathrm{K}_{\iota, \sigma}^{w}$, we consider self-dual perverse sheaves $B$ only and do not take the bilinear form $B \simeq D_{X} B(-w)$ as part of the data. Alternatively, we can also include the form and consider the Grothendieck group GS of symmetric spaces in $\operatorname{Perv}_{\iota}^{\{w\}}$ (equipped with the duality $D_{X}(-w)$ and the evaluation map modified by a factor of $\left.(-1)^{w} \sigma\right)$. The Grothendieck-Witt group $\mathrm{GW}$ is a quotient of GS, equipped with a homomorphism $\mathrm{GW} \rightarrow \mathrm{K}_{\iota}^{w}$. We refer the reader to [Quebbemann et al. 1979, page 280; Schlichting 2010, Section 2.2] for the definition of the Grothendieck-Witt group of an abelian category with duality (generalizing Quillen's definition [1971, Section 5.1] for representations). In our situation, the canonical maps

$$
\mathrm{GS} \rightarrow \mathrm{GW} \rightarrow \mathrm{K}_{l, \sigma}^{w}
$$

are isomorphisms. In fact, by definition, $\mathrm{K}_{l, \sigma}^{w}$ is the image of GW. Moreover, since we work over the algebraically closed field $\overline{\mathbb{Q}}_{\ell}$, symmetric spaces with isomorphic underlying objects are isometric [Quebbemann et al. 1979, Applications 3.4(3)].

We now consider preservation of $\mathrm{K}_{\iota, \sigma}$ and $\mathrm{K}_{\iota, \mathrm{d}}$ by cohomological operations. The preservation of $\mathrm{K}_{\iota, \mathrm{d}}$ is equivalent to the commutation with the twisted dualizing map $\bar{D}_{\iota}$. The main result of this section is the following generalization of Theorem 1.9 .

Theorem 4.2.5. Let $f: X \rightarrow Y$ be a morphism between Deligne-Mumford stacks of finite inertia and finite presentation over $\mathbb{F}_{q}$. Then Grothendieck's six operations 
induce maps

$$
\begin{gathered}
-\otimes-, \mathscr{H o m}(-,-): \mathrm{K}_{\iota, \sigma}\left(X, \overline{\mathbb{Q}}_{\ell}\right) \times \mathrm{K}_{\iota, \sigma^{\prime}}\left(X, \overline{\mathbb{Q}}_{\ell}\right) \rightarrow \mathrm{K}_{\iota, \sigma \sigma^{\prime}}\left(X, \overline{\mathbb{Q}}_{\ell}\right), \\
f^{*}, f^{!}: \mathrm{K}_{\iota, \sigma}\left(Y, \overline{\mathbb{Q}}_{\ell}\right) \rightarrow \mathrm{K}_{\iota, \sigma}\left(X, \overline{\mathbb{Q}}_{\ell}\right), \\
f_{*}, f_{!}: \mathrm{K}_{\iota, \sigma}\left(X, \overline{\mathbb{Q}}_{\ell}\right) \rightarrow \mathrm{K}_{\iota, \sigma}\left(Y, \overline{\mathbb{Q}}_{\ell}\right) .
\end{gathered}
$$

Moreover, Grothendieck's six operations on $\mathrm{K}_{l}^{\mathbb{Z}}$ commute with the twisted dualizing $\operatorname{map} \bar{D}_{\iota}$.

The proof will be given in the next section. We now make a list of pure cases in which the preservation has already been established. Most items of the list below follow from Remark 3.2.8.

Remark 4.2.6 (preservation of $\mathrm{K}_{\iota, \sigma}$, pure cases). Let $f: X \rightarrow Y$ be a morphism, and let $w, w^{\prime} \in \mathbb{Z}$.

(1) $D_{X}$ carries $\mathrm{K}_{l, \sigma}^{w}\left(X, \overline{\mathbb{Q}}_{\ell}\right)$ to $\mathrm{K}_{l, \sigma}^{-w}\left(X, \overline{\mathbb{Q}}_{\ell}\right)$.

(2) If $f$ is smooth, then $f^{*}$ preserves $\mathrm{K}_{l, \sigma}^{w}$.

(3) If $f$ is an open immersion, then $f_{! *}$ preserves $\mathbf{K}_{l, \sigma}^{w}$.

(4) The functor $-\nabla-$ carries $\mathrm{K}_{l, \sigma}^{w}\left(X, \overline{\mathbb{Q}}_{\ell}\right) \times \mathrm{K}_{l, \sigma^{\prime}}^{w^{\prime}}\left(X^{\prime}, \overline{\mathbb{Q}}_{\ell}\right)$ to $\mathrm{K}_{l, \sigma \sigma^{\prime}}^{w+w^{\prime}}\left(X \times X^{\prime}, \overline{\mathbb{Q}}_{\ell}\right)$, and the functor $(-)^{\bigotimes m}, m \geq 0$, carries $\mathrm{K}_{l, \sigma}^{w}\left(X, \overline{\mathbb{Q}}_{\ell}\right)$ to $\mathrm{K}_{l, \sigma^{m}}^{m w}\left(\left[X^{m} / \mathfrak{S}_{m}\right], \overline{\mathbb{Q}}_{\ell}\right)$. For the latter we use Proposition 3.2.14.

(5) Assume that $f$ is a closed immersion and let $A \in \mathrm{K}\left(X, \overline{\mathbb{Q}}_{\ell}\right)$. Then we have $A \in \mathrm{K}_{l, \sigma}^{w}\left(X, \overline{\mathbb{Q}}_{\ell}\right)$ if and only if $f_{*} A \in \mathrm{K}_{l, \sigma}^{w}\left(Y, \overline{\mathbb{Q}}_{\ell}\right)$.

(6) Assume that $f$ is proper. If $f$ is projective or $Y$ has finite inertia, then $f_{*}$ preserves $\mathrm{K}_{\iota, \sigma}^{w}$, by Proposition 3.2.12 and Theorem 3.2.3.

Similar properties hold for $\mathrm{K}_{\iota, \mathrm{d}}$.

The Zariski local nature of $\mathrm{K}_{l, \sigma}^{w}$ will be used in the proof of Theorem 4.2.5. It follows from the Zariski local nature of $\sigma$-self-dual perverse sheaves (Proposition 2.2.1). It also follows from Remark 4.2.6(3) and Lemma 4.1.3.

Remark 4.2.7 (Zariski local nature). Let $\left(X_{\alpha}\right)_{\alpha \in I}$ be a Zariski open covering of $X$ and let $A \in \mathrm{K}\left(X, \overline{\mathbb{Q}}_{\ell}\right)$. Then $A \in \mathrm{K}_{l, \sigma}^{w}\left(X, \overline{\mathbb{Q}}_{\ell}\right)$ if and only if $A \mid X_{\alpha} \in \mathrm{K}_{l, \sigma}^{w}\left(X_{\alpha}, \overline{\mathbb{Q}}_{\ell}\right)$ for every $\alpha$. The same holds for $\mathrm{K}_{\iota, \mathrm{d}}$.

We now turn to consequences of Theorem 4.2.5. The ring part of the next two corollaries follows from the two assertions of Theorem 4.2.5 applied to $a_{X}^{*}$ (recall $\left.a_{X}: X \rightarrow \operatorname{Spec}\left(\mathbb{F}_{q}\right)\right)$ and $-\otimes-$. For the $\lambda$-ring part, we apply Remark 4.2.6(4) to the map (-) $)^{\square m}$ and Theorem 4.2.5 to the maps $\Delta^{*}$ and $p_{*}$ in the definition of $\lambda^{m}$ in Construction 4.1.1. 
Corollary 4.2.8. Assume that $X$ has finite inertia. Then $\mathrm{K}_{\iota, 1}\left(X, \overline{\mathbb{Q}}_{\ell}\right)$ is a $\lambda$-subring of $\mathrm{K}\left(X, \overline{\mathbb{Q}}_{\ell}\right)$. In particular, $\mathrm{K}_{\iota, 1}\left(X, \overline{\mathbb{Q}}_{\ell}\right)$ contains the class $\left[\overline{\mathbb{Q}}_{\ell}\right]$ of the constant sheaf $\overline{\mathbb{Q}}_{\ell}$ on $X$.

Corollary 4.2.9. Assume that $X$ has finite inertia. Then $\bar{D}_{\iota, X}: \mathrm{K}_{\iota}^{\mathbb{Z}}\left(X, \overline{\mathbb{Q}}_{\ell}\right) \rightarrow$ $\mathrm{K}_{l}^{\mathbb{Z}}\left(X, \overline{\mathbb{Q}}_{\ell}\right)$ is a $\lambda$-ring homomorphism. In particular, $\bar{D}_{\iota, X}\left[\overline{\mathbb{Q}}_{\ell}\right]=\left[\overline{\mathbb{Q}}_{\ell}\right]$.

Another consequence of Theorem 4.2.5 is the following pointwise characterization of $\mathrm{K}_{\iota, \mathrm{d}}$ and $\mathrm{K}_{\iota, \mathrm{sd}}$. We let $\mathrm{K}_{\iota, \bar{l}}\left(X, \overline{\mathbb{Q}}_{\ell}\right) \subseteq \mathrm{K}\left(X, \overline{\mathbb{Q}}_{\ell}\right)^{2}\left(\operatorname{resp} . \mathrm{K}_{\iota, \mathbb{R}}\left(X, \overline{\mathbb{Q}}_{\ell}\right) \subseteq\right.$ $\left.\mathrm{K}\left(X, \overline{\mathbb{Q}}_{\ell}\right)\right)$ denote the subgroup consisting of elements $\left(A, A^{\prime}\right)$ (resp. $A$ ) such that, for every morphism $x: \operatorname{Spec}\left(\mathbb{F}_{q^{m}}\right) \rightarrow X$ and every geometric point $\bar{x}$ above $x$, we have

$$
\iota \operatorname{tr}\left(\operatorname{Frob}_{x}, A_{\bar{x}}\right)=\bar{\imath} \operatorname{tr}\left(\text { Frob }_{x}, A_{\bar{x}}^{\prime}\right) \quad\left(\text { resp. } \iota \operatorname{tr}\left(\operatorname{Frob}_{x}, A_{\bar{x}}\right) \in \mathbb{R}\right) .
$$

The notation $\mathrm{K}_{\iota, \bar{l}}$ and $\mathrm{K}_{\iota, \mathbb{R}}$ will only be used in Corollary 4.2.10 and Remark 4.2.11.

Corollary 4.2.10. Assume that $X$ has finite inertia. Let $A \in \mathrm{K}_{l}^{\mathbb{Z}}\left(X, \overline{\mathbb{Q}}_{\ell}\right)$. Then for every $m \geq 1$, every morphism $x: \operatorname{Spec}\left(\mathbb{F}_{q^{m}}\right) \rightarrow X$, and every geometric point $\bar{x}$ above $x$, we have

$$
\iota \operatorname{tr}\left(\operatorname{Frob}_{x}, A_{\bar{x}}\right)=\bar{\imath} \operatorname{tr}\left(\operatorname{Frob}_{x},\left(\bar{D}_{\iota, X} A\right)_{\bar{x}}\right) .
$$

Moreover, $\mathrm{K}_{\iota, \mathrm{d}}\left(X, \overline{\mathbb{Q}}_{\ell}\right)=\mathrm{K}_{\iota, \bar{l}}\left(X, \overline{\mathbb{Q}}_{\ell}\right) \cap \mathrm{K}_{l}^{\mathbb{Z}}\left(X, \overline{\mathbb{Q}}_{\ell}\right)^{2}$. In particular, $\mathrm{K}_{\iota, \mathrm{sd}}\left(X, \overline{\mathbb{Q}}_{\ell}\right)=$ $\mathrm{K}_{\iota, \mathbb{R}} \cap \mathrm{K}_{\iota}^{\mathbb{Z}}\left(X, \overline{\mathbb{Q}}_{\ell}\right)$.

Proof. By the second assertion of Theorem 4.2.5 applied to $x^{*}$, we see that $\bar{D}_{\iota, \operatorname{Spec}\left(\mathbb{F}_{q}\right)} x^{*} A=x^{*} \bar{D}_{\iota, X} A$. Therefore, (4-2-1) in Example 4.2.2 implies (4-2-2). It follows that $\mathrm{K}_{\iota, \mathrm{d}}\left(X, \overline{\mathbb{Q}}_{\ell}\right) \subseteq \mathrm{K}_{\iota, \bar{l}}\left(X, \overline{\mathbb{Q}}_{\ell}\right) \cap \mathrm{K}_{l}^{\mathbb{Z}}\left(X, \overline{\mathbb{Q}}_{\ell}\right)^{2}$. The inclusion in the other direction follows from the injectivity of the homomorphism $\mathrm{K}\left(X, \overline{\mathbb{Q}}_{\ell}\right) \rightarrow$ $\operatorname{Map}\left(\bigsqcup_{m \geq 1}\left|X\left(\mathbb{F}_{q^{m}}\right)\right|, \overline{\mathbb{Q}}_{\ell}\right)$ (Lemma 4.1.4). The last assertion of Corollary 4.2.10 follows from the second one.

Remark 4.2.11. Corollary 4.2.10 also follows from [Katz 2005, Parts (1) and (4) of Lemma 1.8.1], which in turn follow from Gabber's theorem on the independence of $\ell$ for middle extensions [Fujiwara 2002, Theorem 3]. By Gabber's theorem on the independence of $\ell$ for Grothendieck's six operations [Fujiwara 2002, Theorem 2] (see [Zheng 2009, 3.2] for a different proof and [Zheng 2009, Proposition 5.8] for the case of stacks), $\mathrm{K}_{\iota, \bar{\iota}}$ and $\mathrm{K}_{\iota, \mathbb{R}}$ in Corollary 4.2.10 are stable under the six operations. Thus the second assertion of Theorem 4.2.5 follows from Gabber's theorems on the independence of $\ell$. We will not use Gabber's theorems on the independence of $\ell$ in our proof of Theorem 4.2.5.

Remark 4.2.12. The pointwise characterization of $\mathrm{K}_{\iota, \mathrm{sd}}$ in Corollary 4.2.10 does not extend to $\mathrm{K}_{\iota, \sigma}$. For instance, if $X$ is regular and geometrically connected and if $f: E \rightarrow X$ is a family of elliptic curves with nonconstant $j$-invariant, then $\mathscr{F}=\mathrm{R}^{1} f_{*} \overline{\mathbb{Q}}_{\ell}$ is a geometrically simple lisse $\overline{\mathbb{Q}}_{\ell}$-sheaf on $X$ by [Deligne 1980, 
Lemme 3.5.5]. Thus we have $[\mathscr{F}] \in \mathrm{K}_{l, 1}^{1}\left(X, \overline{\mathbb{Q}}_{\ell}\right) \backslash \mathrm{K}_{l,-1}^{1}\left(X, \overline{\mathbb{Q}}_{\ell}\right)$, but, for every closed point $x$ of $X$, we have $\left[\mathscr{F}_{x}\right] \in \mathrm{K}_{l, 1}^{1}\left(x, \overline{\mathbb{Q}}_{\ell}\right) \subseteq \mathrm{K}_{l,-1}^{1}\left(x, \overline{\mathbb{Q}}_{\ell}\right)$.

Remark 4.2.13. Let $f: X \rightarrow Y$ be as in Theorem 4.2.5 and let $\mathrm{I}\left(Y, \overline{\mathbb{Q}}_{\ell}\right) \subseteq \mathrm{K}\left(Y, \overline{\mathbb{Q}}_{\ell}\right)$ be the ideal generated by $\left[\overline{\mathbb{Q}}_{\ell}(1)\right]-\left[\overline{\mathbb{Q}}_{\ell}\right]$. A theorem of Laumon [1981] ([Illusie and Zheng 2013, Theorem 3.2] for the case of Deligne-Mumford stacks) states that

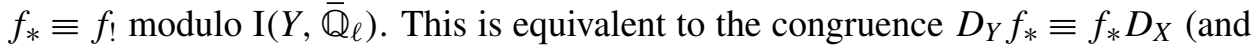
to $\left.D_{Y} f_{!} \equiv f_{!} D_{X}\right)$ modulo $\mathrm{I}\left(Y, \overline{\mathbb{Q}}_{\ell}\right)$. Thus the second assertion of Theorem 4.2.5 can be seen as a refinement of Laumon's theorem.

In the case of $\mathrm{K}_{\iota, \sigma}$, we have the following result on the independence of $(\ell, \iota)$. Let $\ell^{\prime} \neq q$ be a prime number and let $\iota^{\prime}: \overline{\mathbb{Q}}_{\ell^{\prime}} \rightarrow \mathscr{C}$ be an embedding.

Corollary 4.2.14. Assume that $X$ has finite inertia. Let $A \in \mathrm{K}_{l}^{\mathbb{Z}}\left(X, \overline{\mathbb{Q}}_{\ell}\right)$ and let $A^{\prime} \in \mathrm{K}_{l^{\prime}}^{\mathbb{Z}}\left(X, \overline{\mathbb{Q}}_{\ell^{\prime}}\right)$. Assume that $A$ and $A^{\prime}$ are compatible in the sense that, for every morphism $x: \operatorname{Spec}\left(\mathbb{F}_{q^{m}}\right) \rightarrow X$ and every geometric point $\bar{x}$ above $x$, we have

$$
\iota \operatorname{tr}\left(\operatorname{Frob}_{x}, A_{\bar{x}}\right)=\iota^{\prime} \operatorname{tr}\left(\operatorname{Frob}_{x}, A_{\bar{x}}^{\prime}\right) .
$$

Then $A$ belongs to $\mathrm{K}_{\iota, \sigma}\left(X, \overline{\mathbb{Q}}_{\ell}\right)$ if and only if $A^{\prime}$ belongs to $\mathrm{K}_{\iota^{\prime}, \sigma}\left(X, \overline{\mathbb{Q}}_{\ell^{\prime}}\right)$.

Proof. Let $\left(X_{\alpha}\right)_{\alpha \in I}$ be a stratification of $X$. Then we have $A=\sum_{\alpha \in I} j_{\alpha !} j_{\alpha}^{*} A$ and $A^{\prime}=\sum_{\alpha \in I} j_{\alpha} ! j_{\alpha}^{*} A^{\prime}$, where $j_{\alpha}: X_{\alpha} \rightarrow X$ is the immersion. Thus, by Theorem 4.2.5, up to replacing $X$ by a stratum, we may assume that $X$ is regular and $A$ belongs to the subgroup generated by lisse $\overline{\mathbb{Q}}_{\ell}$-sheaves, that is, $A=\sum_{\mathscr{F}} n_{\mathscr{F}}[\mathscr{F}]$, where $\mathscr{F}$ runs over isomorphism classes of simple lisse $\overline{\mathbb{Q}}_{\ell}$-sheaves. For each $\mathscr{F}$ appearing in the decomposition, let $\mathscr{F}^{\prime}$ be the companion of $\mathscr{F}$ [Drinfeld 2012] ([Zheng 2015a] for the case of stacks), namely the simple lisse Weil $\overline{\mathbb{Q}}_{\ell^{\prime}}$-sheaf such that

$$
\iota \operatorname{tr}\left(\operatorname{Frob}_{x}, \mathscr{F}_{\bar{x}}\right)=\iota^{\prime} \operatorname{tr}\left(\operatorname{Frob}_{x}, \mathscr{F}_{\bar{x}}^{\prime}\right)
$$

for all $x$ and $\bar{x}$ as above. Since $A^{\prime}=\sum_{\mathscr{F}} n_{\mathscr{F}}\left[\mathscr{F}^{\prime}\right]$, each $\mathscr{F}^{\prime}$ is an honest $\overline{\mathbb{Q}}_{\ell^{\prime}}$-sheaf. By Corollary 4.2.10, we have $(\bar{D} \mathscr{F})^{\prime}=\bar{D}\left(\mathscr{F}^{\prime}\right)$. Therefore, we may assume that $A=[\mathscr{F}]$ and $A^{\prime}=\left[\mathscr{F}^{\prime}\right]$. In this case, the assertion follows from the symmetry criterion in terms of squares of Frobenius (Proposition 3.2.15).

4.3. Proof of main result. The situation of Theorem 4.2.5 is quite different from that of Gabber's theorem on the independence of $\ell$ [Fujiwara 2002, Theorem 2]. In Gabber's theorem, the preservation by $-\otimes-$ and $f^{*}$ is trivial and the preservation by $f$ ! follows from the Grothendieck trace formula. The key point of Gabber's theorem is thus the preservation by $D_{X}$. The preservation by middle extensions [Fujiwara 2002, Theorem 3] follows from the preservation by the six operations. In Theorem 4.2.5, the stability under each of the six operations is nontrivial, but the preservation by $D_{X}$ and middle extensions is easy. To prove Theorem 4.2.5, we 
will first deduce that $f$ ! preserves $\mathrm{K}_{\iota, \sigma}$ and $\mathrm{K}_{\iota, \mathrm{d}}$ in an important special case from the preservation by middle extensions.

Proposition 4.3.1. Let $X$ be a regular Deligne-Mumford stack of finite presentation over $\mathbb{F}_{q}$ and let $D=\sum_{\alpha \in I} D_{\alpha}$ be a strict normal crossing divisor, with $D_{\alpha}$ regular. Assume that there exists a finite étale morphism $f: Y \rightarrow X$ such that $f^{-1}\left(D_{\alpha}\right)$ is defined globally by $t_{\alpha} \in \Gamma\left(Y, \mathscr{O}_{Y}\right)$ for all $\alpha \in I$. Let $\mathscr{F}$ be a lisse $\overline{\mathbb{Q}}_{\ell}$-sheaf on $U=X-D$, tamely ramified along $D$. Assume that $[\mathscr{F}] \in \mathrm{K}_{l}^{\mathbb{Z}}\left(U, \overline{\mathbb{Q}}_{\ell}\right)$. Then $\bar{D}_{\iota, X}[j ! \mathscr{F}]=j_{!} \bar{D}_{\iota, U}[\mathscr{F}]$ and, if $[\mathscr{F}] \in \mathrm{K}_{\iota, \sigma}\left(U, \overline{\mathbb{Q}}_{\ell}\right),[j ! \mathscr{F}]$ belongs to $\mathrm{K}_{\iota, \sigma}\left(X, \overline{\mathbb{Q}}_{\ell}\right)$, where $j: U \rightarrow X$ is the open immersion.

Proof. We will prove the case of $\mathrm{K}_{\iota, \sigma}$. The case of $\mathrm{K}_{\iota, \mathrm{d}}$ is similar.

We may assume that $f$ is a Galois étale cover of group $G$. Note that, for $g \in G$, we have $g t_{\alpha}=u t_{\alpha}$ for some root of unity $u$ in $k$. We apply the construction of [Deligne 1980, 1.7.9] to our setting as follows. For $J \subseteq I$, let $U_{J}=X-\bigcup_{\beta \in I-J} D_{\beta}$ and let $D_{J}^{*}=\bigcap_{\beta \in J} D_{\beta} \cap U_{J}$. For a locally constant constructible sheaf of sets $\mathscr{G}$ on $U$, tamely ramified along $D$, there exists an integer $n$ invertible in $k$ such that $f^{-1} \mathscr{G}$ extends to $\mathscr{G}^{\prime}$ on the cover $\left(f^{-1} U_{J}\right)\left[t_{\alpha}^{1 / n}\right]_{\alpha \in J}$ of $f^{-1} U_{J}$. We let $\left(f^{-1} \mathscr{G}\right)\left[f^{-1} D_{J}^{*}\right]$ denote the restriction of $\mathscr{G}^{\prime}$ to $D_{J}^{*}$, which is locally constant constructible and equipped with an action of a central extension $G_{J}$ of $G$ by $\mu_{n}^{J}$, compatible with the action of $G$ on $f^{-1} D_{J}^{*}$. Extending this construction to $\overline{\mathbb{Q}}_{\ell}$-sheaves by taking limits, we obtain a lisse $\overline{\mathbb{Q}}_{\ell}$-sheaf $\left(f^{*} \mathscr{F}\right)\left[f^{-1} D_{J}^{*}\right]$ on $f^{-1} D_{J}^{*}$ endowed with an action of a central extension of $G$ by $\hat{\mathbb{Z}}_{L}^{J}(1)$, compatible with the action of $G$ on $f^{-1} D_{J}^{*}$. Here $L$ denotes the set of primes invertible in $k$.

Let us first show that, for all $J \subseteq I$, we have $\left[j_{J}^{*} R j_{*} \mathscr{F}\right] \in \mathrm{K}_{l, \sigma}\left(D_{J}^{*}, \overline{\mathbb{Q}}_{\ell}\right)$, where $j_{J}: D_{J}^{*} \rightarrow X$ is the immersion. We proceed by induction on \#J. The assertion is trivial for $J$ empty, as $j_{\varnothing}=j$. For $J$ nonempty, choose $\beta \in J$. Consider the diagram with Cartesian square

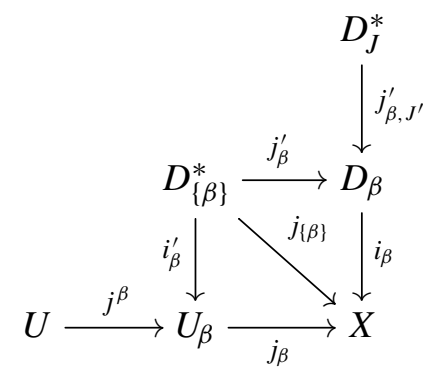

in which $U_{\beta}=X-\bigcup_{\alpha \in I-\{\beta\}} D_{\alpha}, D_{\{\beta\}}=D_{\beta} \cap U_{\beta}$, and $J^{\prime}=J-\{\beta\}$. By [Zheng 2008, Lemme 3.7] (or by direct computation using [Deligne 1980, 1.7.9]), the base change morphism

$$
i_{\beta}^{*} \mathrm{R} j_{*} \mathscr{F} \rightarrow \mathrm{R}_{\beta *}^{\prime} i_{\beta}^{* *} \mathrm{R} j_{*}^{\beta} \mathscr{F}
$$


is an isomorphism, so that

$$
j_{J}^{*} \mathrm{R} j_{*} \mathscr{F} \simeq\left(j_{\beta, J^{\prime}}^{\prime}\right)^{*} i_{\beta}^{*} \mathrm{R} j_{*} \mathscr{F} \simeq\left(j_{\beta, J^{\prime}}^{\prime}\right)^{*} R j_{\beta *}^{\prime} i_{\beta}^{\prime *} \mathrm{R} j_{*}^{\beta} \mathscr{F} .
$$

Since $\# J^{\prime}<\# J$, by the induction hypothesis applied to $j_{\beta}^{\prime}$ and $J^{\prime}$, it suffices to show that the class of $i_{\beta}^{\prime *} \mathrm{R} j_{*}^{\beta} \mathscr{F} \simeq j_{\{\beta\}}^{*} \mathrm{R} j_{*} \mathscr{F}$ is in $\mathrm{K}_{l, \sigma}\left(D_{\{\beta\}}^{*}\right.$, $\left.\overline{\mathbb{Q}}_{\ell}\right)$. For this, we may assume $\mathscr{F}$ l-pure of weight $w \in \mathbb{Z}$. Let $H_{\beta}<\hat{\mathbb{Z}}_{L}(1)^{\{\beta\}}<G_{\{\beta\}}$ be an open subgroup whose action on $V=\left(f^{*} \mathscr{F}\right)\left[f^{-1} D_{\{\beta\}}^{*}\right]$ is unipotent. Let $N: V(1) \rightarrow V$ be the logarithm of this action and let $M$ be the monodromy filtration on $V$. We have $j_{\{\beta\}}^{*} \mathrm{R} j_{*} \in \mathrm{D}^{[0,1]}$,

$$
\begin{aligned}
j_{\{\beta\}}^{*} j_{*} \mathscr{F} & \simeq(\operatorname{Ker}(N)(-1))^{G_{\{\beta\}} / H_{\beta},}, \\
j_{\{\beta\}}^{*} \mathrm{R}^{1} j_{*} \mathscr{F} & \simeq(\operatorname{Coker}(N))^{G_{\{\beta\}} / H_{\beta}}(-1), \\
\operatorname{gr}_{i}^{M}(\operatorname{Ker}(N)(-1)) & \simeq \begin{cases}P_{i}(V, N) & i \leq 0, \\
0 & i>0,\end{cases} \\
\operatorname{gr}_{i}^{M}(\operatorname{Coker}(N)) & \simeq \begin{cases}P_{-i}(V, N)(-i) & i \geq 0, \\
0 & i<0 .\end{cases}
\end{aligned}
$$

By [Deligne 1980, Corollaire 1.8.7, Remarque 1.8.8], $P_{i}(V, N)$ is pure of weight $w+i$ for $i \leq 0$. Moreover, $P_{i}(V, N)$ is $(-1)^{w+i} \sigma$-self-dual by Proposition A.6.8. It follows that $\left[j_{\{\beta\}}^{*} j_{*} \mathscr{F}\right],\left[j_{\{\beta\}}^{*} \mathrm{R}^{1} j_{*} \mathscr{F}\right] \in \mathrm{K}_{\iota, \sigma}$.

Next we show that, if $\mathscr{F}$ is $\iota$-pure of weight $w \in \mathbb{Z}$, then, for all $n \geq 0$ and $J \subseteq I$, we have $\left[j_{J}^{*} \mathrm{R} j_{n *} j_{! *}^{n} \mathscr{F}\right] \in \mathrm{K}_{\iota, \sigma}\left(D_{J}^{*}, \overline{\mathbb{Q}}_{\ell}\right)$. Here $U \stackrel{j^{n}}{\longrightarrow} U_{n} \stackrel{j_{n}}{\longrightarrow} X$ are immersions, where $U_{n}=X-\bigcup_{K \subseteq I, \# K \geq n} D_{K}^{*}$ and $j_{! *}^{n} \mathscr{F}:=\left(j_{! *}^{n}(\mathscr{F}[d])\right)[-d]$, where $d=\operatorname{dim}(X)$ (a function on $\left.\pi_{0}(X)\right)$. The proof is similar to Gabber's proof of the independence of $\ell$ for middle extensions [Fujiwara 2002, Theorem 3]. We proceed by induction on $n$. For $n=0$, we have $U_{0}=U$ and the assertion is shown in the preceding paragraph. For $n \geq 1$, consider the immersions

$$
U_{n}-U_{n-1} \stackrel{i_{n}}{\longrightarrow} U_{n} \stackrel{j_{n-1}^{n}}{\longleftarrow} U_{n-1}
$$

and the distinguished triangle

$$
i_{n *} \mathrm{R} i_{n}^{!} j_{!_{*}}^{n} \mathscr{F} \rightarrow j_{! *}^{n} \mathscr{F} \rightarrow \mathrm{R}\left(j_{n-1}^{n}\right)_{*} j_{! *}^{n-1} \mathscr{F} \rightarrow .
$$

The second and third arrows induce isomorphisms $j_{! *}^{n} \mathscr{F} \stackrel{\sim}{\longrightarrow} \tau^{\leq n-1} \mathrm{R}\left(j_{n-1}^{n}\right)_{*} j_{! *}^{n-1} \mathscr{F}$ and $\tau^{\geq n} \mathrm{R}\left(j_{n-1}^{n}\right)_{*} j_{! *}^{n-1} \mathscr{F} \stackrel{\sim}{\longrightarrow} i_{n *} \mathrm{R} i_{n}^{!} j_{! *}^{n} \mathscr{F}[1]$. By the induction hypothesis, the lefthand side of

$$
\left[i_{n}^{*} \mathrm{R}\left(j_{n-1}^{n}\right)_{*} j_{! *}^{n-1} \mathscr{F}\right]=\left[i_{n}^{*} j_{! *}^{n} \mathscr{F}\right]-\left[\mathrm{R} i{ }_{n}^{!} j_{! *}^{n} \mathscr{F}\right]
$$

belongs to $\mathbf{K}_{\iota, \sigma}$. The first term of the right-hand side belongs to $\bigoplus_{w^{\prime} \leq w+n-1} \mathbf{K}_{\iota}^{w^{\prime}}$ and the second term belongs to $\bigoplus_{w^{\prime} \geq w+n+1} \mathrm{~K}_{l}^{w^{\prime}}$. It follows that both terms belong 
to $\mathrm{K}_{\iota, \sigma}$. Thus, by the preceding paragraph, $\left[j_{J}^{*} R\left(j_{n} i_{n}\right)_{*} \mathrm{R} i_{n}^{!} j_{! *}^{n} \mathscr{F}\right] \in \mathrm{K}_{\iota, \sigma}$. Moreover, by the induction hypothesis, $\left[j_{J}^{*} \mathrm{R}\left(j_{n-1}\right)_{*} j_{! *}^{n-1} \mathscr{F}\right] \in \mathrm{K}_{l, \sigma}$. Therefore, we have $\left[j_{J}^{*} \mathrm{R} j_{n *} j_{! *}^{n} \mathscr{F}\right]=\left[j_{J}^{*} R\left(j_{n} i_{n}\right)_{*} \mathrm{R} i_{n}^{!} j_{! *}^{n} \mathscr{F}\right]+\left[j_{J}^{*} \mathrm{R}\left(j_{n-1}\right)_{*} j_{! *}^{n-1} \mathscr{F}\right] \in \mathrm{K}_{\iota, \sigma}$.

Taking $n=1+\# I$ so that $U_{n}=X$ in the preceding paragraph, we get that $\left[j_{J}^{*} j_{! *} \mathscr{F}\right] \in \mathrm{K}_{\iota, \sigma}\left(D_{J}^{*}, \overline{\mathbb{Q}}_{\ell}\right)$, for $\mathscr{F}$ l-pure and $J \subseteq I$. Here $j_{! *} \mathscr{F}:=\left(j_{! *}(\mathscr{F}[d])\right)[-d]$.

Finally, we show the proposition by induction on \#I. The assertion is trivial for $I$ empty. For $I$ nonempty, we may assume $\mathscr{F}$ l-pure. We have

$$
\left[j_{! *} \mathscr{F}\right]=[j ! \mathscr{F}]+\sum_{\varnothing \neq J \subseteq I}\left[j_{J !} j_{J}^{*} j_{! *} \mathscr{F}\right] .
$$

By the preceding paragraph and the induction hypothesis, for $\varnothing \neq J \subseteq I$, we have $\left[j_{J !} j_{J}^{*} j_{! *} \mathscr{F}\right] \in \mathrm{K}_{\iota, \sigma}\left(X, \overline{\mathbb{Q}}_{\ell}\right)$. Moreover, $\left[j_{! *} \mathscr{F}\right] \in \mathrm{K}_{\iota, \sigma}\left(X, \overline{\mathbb{Q}}_{\ell}\right)$. It follows that $[j ! \mathscr{F}] \in \mathrm{K}_{\iota, \sigma}\left(X, \overline{\mathbb{Q}}_{\ell}\right)$.

Lemma 4.3.2. Let $X$ be a Noetherian Deligne-Mumford stack with separated diagonal. Then there exist a finite group $G$ and a $G$-equivariant dominant open immersion $V \rightarrow W$ of schemes such that the induced morphism $[V / G] \rightarrow[W / G]$ fits into a commutative diagram

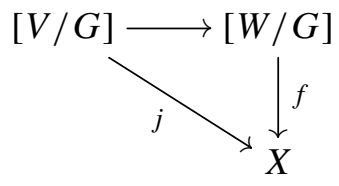

in which $j$ is an open immersion and $f$ is quasifinite, proper, and surjective.

Proof. By [Laumon and Moret-Bailly 2000, 16.6.3], there exists a finite group $G$ acting on a scheme $V$ that fits into a Cartesian square

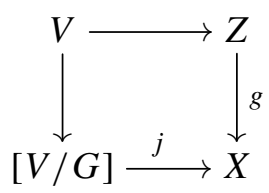

in which $Z$ is a scheme, $g$ is finite surjective, and $j$ is a dense open immersion. It then suffices to take $W$ to be the schematic closure of $V$ in $(Z / X)^{G}$ (fiber product over $X$ of copies of $Z$ indexed by $G$ ) endowed with the action of $G$ by permutation of factors.

Proof of Theorem 4.2.5. We will prove the preservation of $\mathrm{K}_{\iota, \sigma}$. The commutation with $\bar{D}_{\iota}$ is similar.

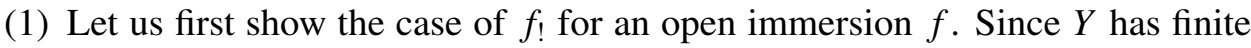
inertia, there exists a Zariski open covering $\left(Y_{\alpha}\right)$ of $Y$ with $Y_{\alpha}$ separated. By the Zariski local nature of $\mathrm{K}_{\iota, \sigma}$ (Remark 4.2.7), we may assume $Y$ separated. We may assume $f$ dominant. 
We proceed by induction on $d=\operatorname{dim} X$. For $d<0$ (i.e., $X=\varnothing$ ), the assertion is trivial. For $d \geq 0$, let $A \in \mathrm{K}_{\iota, \sigma}\left(X, \overline{\mathbb{Q}}_{\ell}\right)$. Note that if $A^{\prime} \in \mathrm{K}\left(X, \overline{\mathbb{Q}}_{\ell}\right)$ is such that the support of $A-A^{\prime}$ has dimension $<d$, then, by the induction hypothesis, to show $f_{!} A \in \mathrm{K}_{\iota, \sigma}\left(Y, \overline{\mathbb{Q}}_{\ell}\right)$, it suffices to show $A^{\prime} \in \mathrm{K}_{\iota, \sigma}\left(X, \overline{\mathbb{Q}}_{\ell}\right)$ and $f_{!} A^{\prime} \in \mathrm{K}_{\iota, \sigma}\left(Y, \overline{\mathbb{Q}}_{\ell}\right)$. This applies in particular to $A^{\prime}=j ! j^{*} A$, where $j: U \rightarrow X$ is a dominant open immersion. In this case $f_{!} A^{\prime}=(f j) ! j^{*} A$. This allows us to shrink $X$.

Applying Lemma 4.3.2 to $Y$, we obtain a finite group $G$, a $G$-equivariant dominant open immersion of schemes $V \rightarrow W$, and a commutative diagram

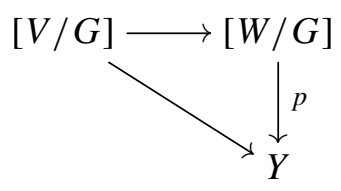

in which $p$ is proper quasifinite surjective, and the oblique arrow is an open immersion. Let $j: U \rightarrow X$, where $U=X \cap[V / G]=\left[V^{\prime} / G\right]$. By the remark above, it suffices to show that $j$ ! and $(f j)$ ! preserve $\mathrm{K}_{\iota, \sigma}$. In the case of $j_{\text {! }}$, up to replacing $Y$ by $X$ and $p$ by its restriction to $X$, we are reduced to the case of $(f j)$ !. Since $(f j)_{!}=f_{!}^{\prime} p_{*}$, where $f^{\prime}: U \rightarrow[W / G]$, we are reduced to the case of $f_{!}^{\prime}$. Thus, changing notation, we are reduced to the case of $f_{!}$, where $f: X=[V / G] \rightarrow[W / G]=Y$ is given by a $G$-equivariant open immersion of schemes $V \rightarrow W$.

The reduction of this case to the case where $V$ is the complement of a $G$-strict normal crossing divisor of $W$ is similar to parts of [Zheng 2009, Section 3]. We may assume $V$ reduced. Shrinking $V$, we may assume $V$ normal and $A=[\mathscr{F}$, where $\mathscr{F}=\mathscr{F}_{\mathscr{O}} \otimes_{\mathscr{O}} \overline{\mathbb{Q}}_{\ell}$, where $\mathscr{F}_{\mathscr{O}}$ is a lisse $\mathscr{O}$-sheaf and $\mathscr{O}$ is the ring of integers of a finite extension of $\mathbb{Q}_{\ell}$. Applying [Zheng 2009, Lemme 3.5], we obtain a $G$-stable dense open subscheme $U$ of $V$ and an equivariant morphism $(u, \alpha):\left(U^{\prime}, G^{\prime}\right) \rightarrow(U, G)$, where $\alpha$ is surjective and $u$ is a Galois étale cover of group $\operatorname{Ker}(\alpha)$ trivializing $\mathscr{F}_{\mathscr{O}} / \mathfrak{m} \mathscr{F}_{\mathscr{O}}$, where $\mathfrak{m}$ is the maximal ideal of $\mathscr{O}$. By Nagata compactification, this can be completed into a commutative diagram

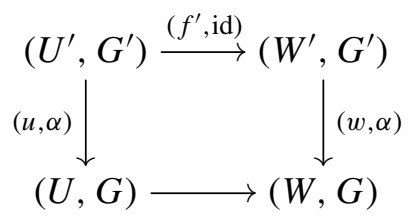

in which $w$ is proper and $f^{\prime}$ is an open immersion. Since $[u / \alpha]$ is an isomorphism and Remark 4.2.6(6) applies to $[w / \alpha]_{*}$, shrinking $X$ and changing notation, we are reduced to the case where $\mathscr{F}_{\mathscr{O}} / \mathfrak{m} \mathscr{F}_{\mathscr{O}}$ is constant on every connected component. We may assume $W$ reduced. Let $k^{\prime}$ be a finite extension of $k$ such that the irreducible components of $W \otimes_{k} k^{\prime}$ are geometrically irreducible. Up to replacing $W$ by $W \otimes_{k} k^{\prime}$ and $G$ by $G \times \operatorname{Gal}\left(k^{\prime} / k\right)$, we may assume that the irreducible components 
of $W$ are geometrically irreducible and that there exists a $G$-equivariant morphism $W \rightarrow \operatorname{Spec}\left(k^{\prime}\right)$. Shrinking $V$, we may assume $V$ regular. Moreover, we may assume that $G$ acts transitively on $\pi_{0}(V)$. Let $V_{0}$ be an irreducible component of $V$ and let $G_{0}$ be the decomposition group. Then $f$ can be decomposed as $X \simeq\left[V_{0} / G_{0}\right] \rightarrow\left[W / G_{0}\right] \stackrel{g}{\longrightarrow}[W / G]=Y$, where $g$ is finite. Changing notation, we may assume $V$ irreducible. Up to replacing $W$ by the closure of $V$, we may assume $W$ irreducible, thus geometrically irreducible.

Applying Gabber's refinement [Zheng 2009, Lemme 3.8] (see also [Vidal 2004, 4.4]) of de Jong's alterations [1997], we obtain a diagram with Cartesian square

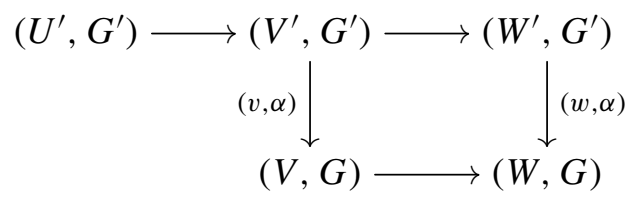

in which $(w, \alpha)$ is a Galois alteration, $W^{\prime}$ is regular quasiprojective over $k$, and $U^{\prime}$ is the complement of a $G^{\prime}$-strict normal crossing divisor of $W^{\prime}$. As $\mathscr{F}$ is lisse and $[V / G],\left[V^{\prime} / G^{\prime}\right]$ are regular, $A^{\prime}=[v / \alpha]^{*} A$ belongs to $\mathrm{K}_{\iota, \sigma}\left(\left[V^{\prime} / G^{\prime}\right], \overline{\mathbb{Q}}_{\ell}\right)$, so that $[v / \alpha]_{*} A$ belongs to $\mathrm{K}_{\iota, \sigma}\left([V / G], \overline{\mathbb{Q}}_{\ell}\right)$. Moreover, the support of $A-[v / \alpha]_{*} A^{\prime}$ has dimension $<d$. Thus it suffices to show that $f_{!}[v / \alpha]_{*} A^{\prime}=[w / \alpha]_{*} f_{!}^{\prime} A^{\prime}$ belongs to $\mathrm{K}_{\iota, \sigma}\left(Y, \overline{\mathbb{Q}}_{\ell}\right)$, where $f^{\prime}:\left[W^{\prime} / G^{\prime}\right] \rightarrow\left[V^{\prime} / G^{\prime}\right]$. Let $j^{\prime}:\left[U^{\prime} / G^{\prime}\right] \rightarrow\left[V^{\prime} / G^{\prime}\right]$. It suffices to show that $j_{!}^{\prime} j^{\prime *} A^{\prime}$ and $\left(f^{\prime} j^{\prime}\right) ! j^{\prime *} A^{\prime}$ belong to $\mathrm{K}_{\iota, \sigma}$. Changing notation, we are reduced to showing $f_{!}[\mathscr{F}] \in \mathrm{K}_{\iota, \sigma}\left([W / G]\right.$, $\left.\overline{\mathbb{Q}}_{\ell}\right)$ for $f:[V / G] \rightarrow[W / G]$, where $V$ is the complement of a $G$-strict normal crossing divisor $D$ of a regular quasiprojective scheme $W$ over $k$ and $\mathscr{F}$ is a lisse sheaf on $[V / G]$ tame along $D$ such that $[\mathscr{F}] \in \mathrm{K}_{\iota, \sigma}\left([V / G], \overline{\mathbb{Q}}_{\ell}\right)$.

Note that $W$ admits a Zariski open covering by $G$-stable affine schemes. Thus, by the Zariski local nature of $\mathrm{K}_{\iota, \sigma}$ (Remark 4.2.7), we may assume $W$ affine. In this case, the assertion is a special case of Proposition 4.3.1. This finishes the proof of the case of $f_{\text {! }}$ for $f$ an open immersion.

(2) Next we establish the general case of $f_{\text {! }}$. Let $\left(X_{\alpha}\right)_{\alpha \in I}$ be a Zariski open covering of $X$ with $X_{\alpha}$ separated. For $J \subseteq I$, let $j_{J}: \bigcap_{\beta \in J} X_{\beta} \rightarrow X$ be the open immersion. Let $A \in \mathrm{K}_{\iota, \sigma}\left(X, \overline{\mathbb{Q}}_{\ell}\right)$. Then, by Lemma 4.1.3, $A=\sum_{\varnothing \neq J \subseteq I}(-1)^{1+\# J} j_{J !} j_{J}^{*} A$. Thus we may assume $X$ separated. Applying Nagata compactification [Conrad et al. 2012] to the morphism $\bar{X} \rightarrow \bar{Y}$ of coarse spaces, we obtain a diagram with Cartesian squares

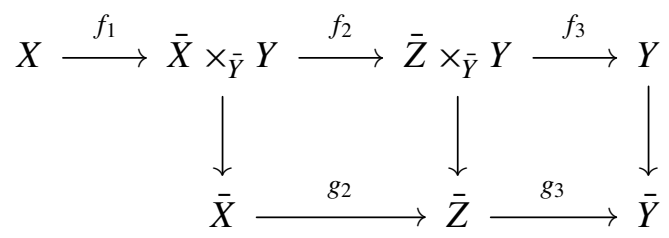


in which $f_{1}$ is proper and quasifinite, $g_{2}$ is an open immersion, and $g_{3}$ is proper. Thus $f_{!}=f_{3 *} f_{2 !} f_{1 *}$ preserves $\mathrm{K}_{\iota, \sigma}$.

The case of $f_{*}=D_{Y} f_{!} D_{X}$ follows immediately.

(3) Next we establish the case of $f^{*}$. The argument is similar to the deduction of the congruence $f^{*} \equiv f^{!}$modulo $\mathrm{I}\left(X, \overline{\mathbb{Q}}_{\ell}\right)$ [Zheng 2015b, Corollary 9.5] from Laumon's theorem, mentioned in Remark 4.2.13. Let $B \in \mathrm{K}_{\iota, \sigma}\left(Y, \overline{\mathbb{Q}}_{\ell}\right)$. If $f$ is a closed immersion, then $B=j_{!} j^{*} B+f_{*} f^{*} B$, where $j$ is the complementary open immersion. It follows that $f_{*} f^{*} B \in \mathrm{K}_{\iota, \sigma}\left(Y, \overline{\mathbb{Q}}_{\ell}\right)$, so that $f^{*} B \in \mathrm{K}_{\iota, \sigma}$. In the general case, let $\left(Y_{\alpha}\right)_{\alpha \in I}$ be a stratification of $Y$ such that each $Y_{\alpha}$ is the quotient stack of an affine scheme by a finite group action. For each $\alpha$, form the Cartesian square

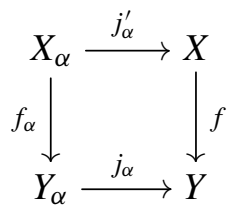

Then $f^{*} B=\sum_{\alpha \in I} f^{*} j_{\alpha !} j_{\alpha}^{*} B=\sum_{\alpha \in I} j_{\alpha !}^{\prime} f_{\alpha}^{*} j_{\alpha}^{*} B$. Thus we may assume $Y=\left[Y^{\prime} / H\right]$, where $Y^{\prime}$ is an affine scheme endowed with an action of a finite group $H$. Similarly, we may assume $X=\left[X^{\prime} / G\right]$, where $X^{\prime}$ is an affine scheme endowed with an action of a finite group $G$. Up to changing $X^{\prime}$ and $G$, we may further assume that $f=\left[f^{\prime} / \gamma\right]$, for $(f, \gamma):\left(X^{\prime}, G\right) \rightarrow\left(Y^{\prime}, H\right)$, by [Zheng 2009, Proposition 5.1]. In this case $f^{\prime}$ can be decomposed into $G$-equivariant morphisms $X^{\prime} \stackrel{i}{\longrightarrow} Z^{\prime} \stackrel{p}{\longrightarrow} Y^{\prime}$ where $i$ is a closed immersion and $p$ is an affine space. Thus $f^{*} \simeq[i / \mathrm{id}]^{*}[p / \gamma]^{*}$ preserves $\mathrm{K}_{\iota, \sigma}$.

The assertions for the other operations follow immediately: $f^{!}=D_{X} f^{*} D_{Y}$, $-\otimes-=\Delta_{X}^{*}(-\otimes-), \mathscr{H} o m(-,-)=D(-\otimes D-)$.

\section{Variant: horizontal complexes}

In this section, let $k$ be a field finitely generated over its prime field. This includes, notably, the case of a number field. Many results in previous sections over finite fields can be generalized to Annette Huber's horizontal complexes [1997], as extended by Sophie Morel [2012], over $k$. In Section 5.1, after briefly reviewing horizontal complexes, we discuss symmetry and decomposition of pure horizontal complexes and prove analogues of results of Section 3.2. In Section 5.2, we discuss symmetry in Grothendieck groups of horizontal complexes and give analogues of results of Section 4. This section stems from a suggestion of Takeshi Saito.

5.1. Symmetry and decomposition of pure horizontal complexes. Let $X$ be a Deligne-Mumford stack of finite presentation over $k$. Huber [1997] (see also [Morel 2012]) defines a triangulated category $\mathrm{D}_{h}^{b}\left(X, \overline{\mathbb{Q}}_{\ell}\right)$ of horizontal complexes. For a finite extension $\Lambda$ of $\mathbb{Z}_{\ell}, \mathrm{D}_{h}^{b}(X, \Lambda)$ is the 2-colimit of the categories $\mathrm{D}_{c}^{b}\left(X_{R}, \Lambda\right)$, 
indexed by triples $\left(R, X_{R}, u\right)$, where $R \subseteq k$ is a subring of finite type over $\mathbb{Z}[1 / \ell]$ such that $k=\operatorname{Frac}(R), X_{R}$ is a Deligne-Mumford stack of finite presentation over $\operatorname{Spec}(R)$, and $u: X \rightarrow X_{R} \otimes_{R} k$ is an isomorphism. We may restrict to $R$ regular and $X_{R}$ flat over $\operatorname{Spec}(R)$. We have Grothendieck's six operations on $\mathrm{D}_{h}^{b}(X, \Lambda)$.

The triangulated category $\mathrm{D}_{h}^{b}\left(X, \overline{\mathbb{Q}}_{\ell}\right)$ is equipped with a canonical $t$-structure and a perverse $t$-structure. We let $\operatorname{Sh}_{h}\left(X, \overline{\mathbb{Q}}_{\ell}\right)$ and $\operatorname{Perv}_{h}\left(X, \overline{\mathbb{Q}}_{\ell}\right)$ denote the respective hearts. The pullback functors via $X \rightarrow X_{R}$ induce a conservative functor $\eta^{*}: \mathrm{D}_{h}^{b}\left(X, \overline{\mathbb{Q}}_{\ell}\right) \rightarrow \mathrm{D}_{c}^{b}\left(X, \overline{\mathbb{Q}}_{\ell}\right) t$-exact for the canonical $t$-structures and the perverse $t$-structures, and compatible with the six operations. Moreover, $\eta^{*}$ induces fully faithful exact functors $\operatorname{Sh}_{h}\left(X, \overline{\mathbb{Q}}_{\ell}\right) \rightarrow \operatorname{Sh}\left(X, \overline{\mathbb{Q}}_{\ell}\right)$ and $\operatorname{Perv}_{h}\left(X, \overline{\mathbb{Q}}_{\ell}\right) \rightarrow \operatorname{Perv}\left(X, \overline{\mathbb{Q}}_{\ell}\right)$ [Morel 2012, Propositions 2.3 and 2.5]. Every object of $\operatorname{Perv}_{h}\left(X, \overline{\mathbb{Q}}_{\ell}\right)$ has finite length.

Remark 5.1.1. The functor $\eta^{*}: \operatorname{Perv}_{h}\left(X, \overline{\mathbb{Q}}_{\ell}\right) \rightarrow \operatorname{Perv}\left(X, \overline{\mathbb{Q}}_{\ell}\right)$ preserves indecomposable objects. By the description of simple objects, the functor also preserves simple objects. Thus, via the functor, $\operatorname{Perv}_{h}\left(X, \overline{\mathbb{Q}}_{\ell}\right)$ can be identified with a full subcategory of $\operatorname{Perv}\left(X, \overline{\mathbb{Q}}_{\ell}\right)$ stable under subquotients. The subcategory is not stable under extensions in general.

By restricting to closed points of $\operatorname{Spec}(R[1 / \ell])$, we get a theory of weights for horizontal complexes. Weight filtration does not always exist, but this will not be a problem for us. The analogue of Remark 3.2.7 for the preservation of pure complexes holds. Moreover, the analogues of [Beilinson et al. 1982, Théorèmes 5.3.8 and 5.4.5] hold for the decomposition of the pullbacks of pure horizontal complexes to $\bar{k}$. In other words, the functor

$$
\bar{\eta}^{*}: \mathrm{D}_{h}^{b}\left(X, \overline{\mathbb{Q}}_{\ell}\right) \stackrel{\eta^{*}}{\longrightarrow} \mathrm{D}_{c}^{b}\left(X, \overline{\mathbb{Q}}_{\ell}\right) \rightarrow \mathrm{D}_{c}^{b}\left(X_{\bar{k}}, \overline{\mathbb{Q}}_{\ell}\right)
$$

obtained by composing $\eta^{*}$ with the pullback functor carries pure complexes to admissible semisimple complexes (Definition 3.3.1). Indeed, both theorems follow from [Beilinson et al. 1982, Proposition 5.1.15(iii)], which has the following analogue, despite the fact that the analogue of [Beilinson et al. 1982, Proposition 5.1.15(ii)] does not hold in general.

Proposition 5.1.2. Let $K, L \in \mathrm{D}_{h}^{b}\left(X, \overline{\mathbb{Q}}_{\ell}\right)$, with $K$ mixed of weights $\leq w$ and $L$ mixed of weights $\geq w$. Then $\left.\operatorname{Ext}^{i}\left(\bar{\eta}^{*} K, \bar{\eta}^{*} L\right)\right)^{\mathrm{Gal}(\bar{k} / k)}=0$ for $i>0$. In particular, the map $\operatorname{Ext}^{i}\left(\eta^{*} K, \eta^{*} L\right) \rightarrow \operatorname{Ext}^{i}\left(\bar{\eta}^{*} K, \bar{\eta}^{*} L\right)$ is zero.

Proof. The second assertion follows from the first one, as the map factors through $E^{i}:=\operatorname{Ext}^{i}\left(\bar{\eta}^{*} K, \bar{\eta}^{*} L\right)^{\mathrm{Gal}(\bar{k} / k)}$. For the first assertion, consider the horizontal complex $\mathscr{E}=\mathrm{R} a_{X *} \mathrm{R} \mathscr{H} o m(K, L)$ on $\operatorname{Spec}(k)$, which has weight $\geq 0$. Therefore, $E^{i} \simeq \Gamma\left(\operatorname{Spec}(k), \mathscr{H}^{i} \mathscr{E}\right)=0$ for $i>0$.

As pure horizontal perverse sheaves are geometrically semisimple, Lemma 2.2.8 on the support decomposition applies (see [Beilinson et al. 1982, Corollaire 5.3.11]). 
The general preservation properties of $\sigma$-self-dual complexes listed in Remark 2.1.4 still hold for $\mathrm{D}_{h}^{b}$. The two-out-of-three property (Proposition 2.2.1) holds for $\sigma$-self-dual horizontal perverse sheaves. The trichotomy for indecomposable horizontal perverse sheaves (Proposition 2.2.3) also holds.

We say that a horizontal complex of $\overline{\mathbb{Q}}_{\ell}$-sheaves $A$ is split if it is a direct sum of shifts of horizontal perverse sheaves, or, in other words, if $A \simeq \bigoplus_{i}\left(P_{\mathscr{H}^{i}} A\right)[-i]$. Definition 3.2.2 can be repeated as follows.

Definition 5.1.3 $\left(\mathrm{D}_{h, \sigma}^{w}\right)$. Let $w \in \mathbb{Z}$. We denote by $\mathrm{D}_{h, \sigma}^{w}\left(X, \overline{\mathbb{Q}}_{\ell}\right) \subseteq \mathrm{Ob}\left(\mathrm{D}_{h}^{b}\left(X, \overline{\mathbb{Q}}_{\ell}\right)\right)$ $\left(\right.$ resp. $\left.\mathrm{D}_{h, \mathrm{sd}}^{w}\left(X, \overline{\mathbb{Q}}_{\ell}\right) \subseteq \mathrm{Ob}\left(\mathrm{D}_{c}^{b}\left(X, \overline{\mathbb{Q}}_{\ell}\right)\right)\right)$ the subset consisting of split pure horizontal complexes $A$ of weight $w$ such that ${ }^{p_{H^{i}} A} A$ is $(-1)^{w+i} \sigma$-self-dual (resp. self-dual) with respect to $K_{X}(-w-i)$ for all $i$. We denote by $\mathrm{D}_{h, \mathrm{~d}}^{w}\left(X, \overline{\mathbb{Q}}_{\ell}\right) \subseteq$ $\operatorname{Ob}\left(\mathrm{D}_{h}^{b}\left(X, \overline{\mathbb{Q}}_{\ell}\right) \times \mathrm{D}_{h}^{b}\left(X, \overline{\mathbb{Q}}_{\ell}\right)\right)$ the subset consisting of pairs $(A, B)$ of split pure horizontal complexes of weight $w$ such that ${ }^{p} \mathscr{H}^{i} A$ is isomorphic to $\left(D_{X}{ }^{p^{i}}{ }^{i} B\right)(-w-i)$ for all $i$.

The analogue of Remark 3.2.8 holds for the preservation of $\mathrm{D}_{h, \sigma}^{w}$ and $\mathrm{D}_{h, \mathrm{~d}}^{w}$. The two-out-of-three property, an analogue of Remark 3.2.9, also holds for $\mathrm{D}_{h, \sigma}^{w}$ and $\mathrm{D}_{h, \mathrm{~d}}^{w}$. We have the following analogue of Proposition 3.2.14, which holds with the same proof as before, and a similar result for $\mathrm{D}_{h, \mathrm{~d}}^{w}$.

Proposition 5.1.4. Let $m \geq 0$. Let $A$ be a mixed horizontal complex on $X$ such that, for all $n \in \mathbb{Z}, P^{p} \mathscr{H}^{n} A$ admits a weight filtration $W$, and such that $\mathrm{gr}_{w}^{W} p_{\mathscr{H}^{n}} A$ is $(-1)^{w} \sigma$-self-dual with respect to $K_{X}(-w)$ for all $w \in \mathbb{Z}$. Then, for all $n \in \mathbb{Z}$, $p_{\mathscr{H}^{n}}\left(A^{\bigotimes m}\right)$ admits a weight filtration $W$, and $\mathrm{gr}_{w}^{W} p_{\mathscr{H}^{n}}\left(A^{\bigotimes m}\right)$ is $(-1)^{w} \sigma^{m}$-self-dual with respect to $K_{\left[X^{m} / \mathfrak{S}_{m}\right]}(-m w)$ for all $w \in \mathbb{Z}$. Moreover, the functor $(-)^{\bigotimes m}$ carries $\mathrm{D}_{h, \sigma}^{w}\left(X, \overline{\mathbb{Q}}_{\ell}\right)$ to $\mathrm{D}_{h, \sigma^{m}}^{m w}\left(\left[X^{m} / \mathfrak{S}_{m}\right], \overline{\mathbb{Q}}_{\ell}\right)$.

We have the following analogues of Theorem 3.2.3 and Corollary 3.2.5, which hold with the same proofs as before.

Theorem 5.1.5. Let $f: X \rightarrow Y$ be a proper morphism of Deligne-Mumford stacks of finite presentation over $k$. Assume that $Y$ has finite inertia. Then $\mathrm{R} f_{*}$ preserves $\mathrm{D}_{h, \sigma}^{w}$ and $\mathrm{D}_{h, \mathrm{~d}}^{w}$.

Corollary 5.1.6. Assume that $Y$ has finite inertia. Then $R f_{*}$ preserves split pure complexes of weight $w$.

The analogue of Corollary 3.2.4 also holds for $\mathrm{D}_{h, \mathrm{sd}}^{b}$.

Theorem 1.8 is a special case of Theorem 5.1.5. Applying it to $a_{X}$, we obtain Theorem 1.1. Indeed, as we remarked in the Introduction, in Theorem 1.1 we may assume that $k$ is finitely generated over its prime field. The horizontal perverse sheaf $\mathrm{IC}_{X}$, pure of weight $d$, is $(-1)^{d}$-self-dual with respect to $K_{X}(-d)$ by Example 2.1.6, so $\mathrm{IC}_{X}[-d] \in \mathrm{D}_{h, 1}^{0}$, hence $\mathrm{R} a_{X *} \mathrm{IC}_{X}[-d] \in \mathrm{D}_{h, 1}^{0}$ by Theorem 1.8 , which proves Theorem 1.1. 
5.2. Symmetry in Grothendieck groups of horizontal complexes. We let $X$ be a Deligne-Mumford stack of finite presentation over $k$, and we let $\mathrm{K}_{h}\left(X, \overline{\mathbb{Q}}_{\ell}\right)$ denote the Grothendieck group of $\mathrm{D}_{h}^{b}\left(X, \overline{\mathbb{Q}}_{\ell}\right)$, which is a free abelian group generated by the isomorphism classes of simple horizontal perverse $\overline{\mathbb{Q}}_{\ell}$-sheaves. The functor $\eta^{*}$ induces an injection $\mathrm{K}_{h}\left(X, \overline{\mathbb{Q}}_{\ell}\right) \rightarrow \mathrm{K}\left(X, \overline{\mathbb{Q}}_{\ell}\right)$, which identifies $\mathrm{K}_{h}\left(X, \overline{\mathbb{Q}}_{\ell}\right)$ with a $\lambda$-subring of $\mathrm{K}\left(X, \overline{\mathbb{Q}}_{\ell}\right)$. The operations on Grothendieck groups in Construction 4.1.1 and Remark 4.1.2 induce operations on $\mathrm{K}_{h}$.

For $w \in \mathbb{Z}$, we let $\mathrm{K}_{h}^{w}\left(X, \overline{\mathbb{Q}}_{\ell}\right) \subseteq \mathrm{K}_{h}\left(X, \overline{\mathbb{Q}}_{\ell}\right)$ denote the subgroup generated by pure horizontal perverse sheaves of weight $w$ on $X$, and we let $\mathrm{K}_{h}^{\mathbb{Z}}\left(X, \overline{\mathbb{Q}}_{\ell}\right)=$ $\bigoplus_{w \in \mathbb{Z}} \mathrm{K}_{h}^{w}\left(X, \overline{\mathbb{Q}}_{\ell}\right) \subseteq \mathrm{K}_{h}\left(X, \overline{\mathbb{Q}}_{\ell}\right)$. The analogue of Lemma 4.1 .8 holds, which further justifies the definition of the map $f_{! *}$ in Remark 4.1.2.

We repeat Definition 4.2.1 as follows.

Definition 5.2.1 $\left(\mathrm{K}_{h, \sigma}\right)$. We let $\mathrm{K}_{h, \sigma}^{w}\left(X, \overline{\mathbb{Q}}_{\ell}\right) \subseteq \mathrm{K}_{h}^{w}\left(X, \overline{\mathbb{Q}}_{\ell}\right)\left(\operatorname{resp} . \mathrm{K}_{h, \mathrm{sd}}^{w}\left(X, \overline{\mathbb{Q}}_{\ell}\right) \subseteq\right.$ $\left.\mathrm{K}_{h}^{w}\left(X, \overline{\mathbb{Q}}_{\ell}\right)\right)$, for $w \in \mathbb{Z}$, be the subgroup generated by $[B]$, for $B$ perverse, $\iota$-pure of weight $w$, and $(-1)^{w} \sigma$-self-dual (resp. self-dual) with respect to $K_{X}(-w)$. We set

$$
\mathrm{K}_{h, \sigma}\left(X, \overline{\mathbb{Q}}_{\ell}\right)=\bigoplus_{w \in \mathbb{Z}} \mathrm{K}_{h, \sigma}^{w}\left(X, \overline{\mathbb{Q}}_{\ell}\right) \quad\left(\operatorname{resp} . \mathrm{K}_{\iota, \mathrm{sd}}\left(X, \overline{\mathbb{Q}}_{\ell}\right)=\bigoplus_{w \in \mathbb{Z}} \mathrm{K}_{h, \mathrm{sd}}^{w}\left(X, \overline{\mathbb{Q}}_{\ell}\right)\right) .
$$

We define the twisted dualizing map

$$
\bar{D}_{h, X}: \mathrm{K}_{h}^{\mathbb{Z}}\left(X, \overline{\mathbb{Q}}_{\ell}\right) \rightarrow \mathrm{K}_{h}^{\mathbb{Z}}\left(X, \overline{\mathbb{Q}}_{\ell}\right)
$$

to be the direct sum of the group automorphisms $\bar{D}_{h, X}^{w}: \mathrm{K}_{h}^{w}\left(X, \overline{\mathbb{Q}}_{\ell}\right) \rightarrow \mathrm{K}_{h}^{w}\left(X, \overline{\mathbb{Q}}_{\ell}\right)$ sending $[A]$ to $\left[\left(D_{X} A\right)(-w)\right]$. We let $\mathrm{K}_{h, \mathrm{~d}}^{w}\left(X, \overline{\mathbb{Q}}_{\ell}\right) \subseteq \mathrm{K}_{h}^{w}\left(X, \overline{\mathbb{Q}}_{\ell}\right)^{2}$ denote the graph of $\bar{D}_{h, X}^{w}$ and set

$$
\mathrm{K}_{h, \mathrm{~d}}\left(X, \overline{\mathbb{Q}}_{\ell}\right)=\bigoplus_{n \in Z} \mathrm{~K}_{h, \mathrm{~d}}^{w}\left(X, \overline{\mathbb{Q}}_{\ell}\right)
$$

The subgroup $\mathrm{K}_{\text {orth }}\left(X, \overline{\mathbb{Q}}_{\ell}\right)$ in the Introduction is $\mathrm{K}_{h, 1}\left(X, \overline{\mathbb{Q}}_{\ell}\right)$. If $k$ is a finite field, $\mathrm{K}_{h, \sigma}\left(X, \overline{\mathbb{Q}}_{\ell}\right)$ is the intersection $\bigcap_{\iota} \mathrm{K}_{\iota, \sigma}\left(X, \overline{\mathbb{Q}}_{\ell}\right)$ of the subgroups $\mathrm{K}_{\iota, \sigma}$ of Definition 4.2.1, where $\iota$ runs over embeddings $\overline{\mathbb{Q}}_{\ell} \hookrightarrow \mathbb{C}$.

The analogue of Remark 4.2.3 holds for the definition of $\mathrm{K}_{h, \sigma}, \mathrm{K}_{h, \mathrm{sd}}$, and $\mathrm{K}_{h, \mathrm{~d}}$. The analogues of Remarks 4.2.6 and 4.2.7 hold for the preservation and Zariski local nature of $\mathrm{K}_{h, \sigma}$ and $\mathrm{K}_{h, \mathrm{~d}}$ with the same proofs.

The following analogue of Theorem 4.2.5 holds with the same proof. In particular, the analogue of Proposition 4.3.1 holds.

Theorem 5.2.2. Let $X$ and $Y$ be Deligne-Mumford stacks of finite inertia and finite presentation over $k$ and let $f: X \rightarrow Y$ be a morphism. Then Grothendieck's six 
operations induce maps

$$
\begin{gathered}
-\otimes-, \mathscr{H o m}(-,-): \mathrm{K}_{h, \sigma}\left(X, \overline{\mathbb{Q}}_{\ell}\right) \times \mathrm{K}_{h, \sigma^{\prime}}\left(X, \overline{\mathbb{Q}}_{\ell}\right) \rightarrow \mathrm{K}_{h, \sigma \sigma^{\prime}}\left(X, \overline{\mathbb{Q}}_{\ell}\right), \\
f^{*}, f^{!}: \mathrm{K}_{h, \sigma}\left(Y, \overline{\mathbb{Q}}_{\ell}\right) \rightarrow \mathrm{K}_{h, \sigma}\left(X, \overline{\mathbb{Q}}_{\ell}\right), \\
f_{*}, f_{!}: \mathrm{K}_{h, \sigma}\left(X, \overline{\mathbb{Q}}_{\ell}\right) \rightarrow \mathrm{K}_{h, \sigma}\left(Y, \overline{\mathbb{Q}}_{\ell}\right) .
\end{gathered}
$$

Moreover, Grothendieck's six operations on $\mathrm{K}_{h}^{\mathbb{Z}}$ commute with the twisted dualizing map $\bar{D}_{h}$.

The analogues of Corollaries 4.2.8 and 4.2.9 hold. The relationship with Laumon's theorem (Remark 4.2.13) also holds.

Theorem 1.9 is a special case of Theorem 5.2.2.

\section{Appendix: Symmetry and duality in categories}

In the appendix, we collect some general symmetry properties in categories with additional structures. The tensor product equips the derived category of $\ell$-adic sheaves with a symmetric structure. We discuss symmetry of pairings in symmetric categories in Section A.1. The category of perverse sheaves is not stable under tensor product, but is equipped with a duality functor. We study symmetry in categories with duality in Sections A.2 and A.3. We discuss the relation of the two points of view in Section A.4. We then study the effects of translation on symmetry in Section A.5; these results are applied in the main text to the Lefschetz pairing. In Section A.6, we study symmetry of primitive parts under a nilpotent operator; these results are applied in the main text to the monodromy operator. The results of the appendix are formal but are used in the main text. The presentation here is influenced by [Quebbemann et al. 1979], [Riou 2014, Section 12], and [Schlichting 2010]. Recall that $\sigma, \sigma^{\prime} \in\{ \pm 1\}$.

A.1. Symmetric categories. In this subsection, we discuss symmetry of pairings in symmetric categories.

Definition A.1.1 (symmetric category). A symmetric category is a category $\mathscr{C}$ endowed with a bifunctor $-\otimes-: \mathscr{C} \times \mathscr{C} \rightarrow \mathscr{C}$ and a natural isomorphism (called the symmetry constraint) $c_{A B}: A \otimes B \rightarrow B \otimes A$, for objects $A$ and $B$ of $\mathscr{C}$, satisfying $c_{A B}^{-1}=c_{B A}$. We say that the symmetric category $\mathscr{C}$ is closed if for every object $A$ of $\mathscr{D}$, the functor $-\otimes A: \mathscr{C} \rightarrow \mathscr{C}$ admits a right adjoint, which we denote by $\mathscr{H}$ om $(A,-)$.

In our applications, we mostly encounter symmetric monoidal categories (see, for example, [Mac Lane 1998, Section VII.7] for the definition), but the associativity and unital constraints are mostly irrelevant to the results of this article.

To deal with signs, we need the following additive variant of Definition A.1.1. 
Definition A.1.2. A symmetric additive category is a symmetric category $(\mathscr{D}, \otimes)$ such that $\mathscr{D}$ is an additive category and $-\otimes-: \mathscr{D} \times \mathscr{D} \rightarrow \mathscr{D}$ is an additive bifunctor (namely, a bifunctor additive in each variable). A closed symmetric additive category is a closed symmetric category $(\mathscr{D}, \otimes)$ such that $\mathscr{D}$ is an additive category.

A closed symmetric additive category is necessarily a symmetric additive category and the internal Hom functor $\mathscr{H} O m(-,-): \mathscr{D}^{\mathrm{op}} \times \mathscr{D} \rightarrow \mathscr{D}$ is an additive bifunctor.

Definition A.1.3. Let $(\mathscr{C}, \otimes, c)$ be a symmetric category. Assume that $\mathscr{C}$ is an additive category if $\sigma=-1$. Let $A, B, K$ be objects of $\mathscr{C}$.

(1) We define the transpose of a pairing $g: B \otimes A \rightarrow K$ to be the composite

$$
g^{T}: A \otimes B \stackrel{c}{\longrightarrow} B \otimes A \stackrel{g}{\longrightarrow} K .
$$

We call $\sigma g^{T}$ the $\sigma$-transpose of $g$.

(2) We say that a pairing $f: A \otimes A \rightarrow K$ is $\sigma$-symmetric if $f=\sigma f^{T}$.

We have $\left(g^{T}\right)^{T}=g$. We will often say "symmetric" instead of "1-symmetric".

Note that, for a pair of pairings $f: A \otimes B \rightarrow K$ and $g: B \otimes A \rightarrow K$ in a symmetric additive category, $(2 f, 2 g)$ is a sum of a pair of 1-transposes and a pair of -1-transposes:

$$
(2 f, 2 g)=\left(f+g^{T}, g+f^{T}\right)+\left(f-g^{T}, g-f^{T}\right) .
$$

Remark A.1.4. Let $(\mathscr{C}, \otimes, c)$ be a symmetric category such that $\mathscr{C}$ is an additive category. Then $(\mathscr{C}, \otimes,-c)$ is another symmetric category. The -1 -transpose in $(\mathscr{C}, \otimes, c)$ of a pairing $g: A \otimes B \rightarrow K$ is the transpose in $(\mathscr{C}, \otimes,-c)$ of $g$.

Next we consider effects of functors on symmetry.

Definition A.1.5. Let $\mathscr{C}$ and $\mathscr{D}$ be symmetric categories. A right-lax symmetric functor (resp. symmetric functor) from $\mathscr{C}$ to $\mathscr{D}$ is a functor $G: \mathscr{C} \rightarrow \mathscr{D}$ endowed with a natural transformation (resp. natural isomorphism) of functors $\mathscr{C} \times \mathscr{C} \rightarrow \mathscr{D}$ given by morphisms $G(A) \otimes G(B) \rightarrow G(A \otimes B)$ in $\mathscr{D}$ for objects $A, B$ of $\mathscr{C}$, such that the following diagram commutes:

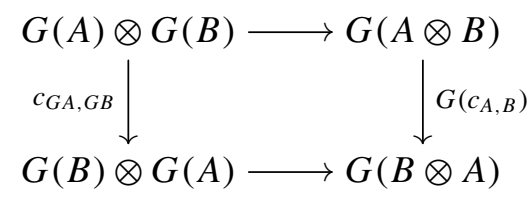

Between symmetric monoidal categories, one has the notions of symmetric monoidal functors and lax symmetric monoidal functors, which are compatible with the associativity constraints and unital constraints. In our applications we will need to consider symmetric functors between symmetric monoidal categories that are not symmetric monoidal functors. For example, if $f$ is an open immersion, 
then $f$ ! is a symmetric functor compatible with the associativity constraint, but not compatible with the unital constraints except in trivial cases. Again we emphasize that the compatibility with the associativity and unital constraints is irrelevant to the results in this article.

Example A.1.6. Let $\mathscr{C}$ and $\mathscr{D}$ be symmetric categories. Let $F: \mathscr{C} \rightarrow \mathscr{D}$ be a functor admitting a right adjoint $G: \mathscr{D} \rightarrow \mathscr{C}$. Then every symmetric structure on $F$ induces a right-lax symmetric structure on $G$, given by the morphism $G(A) \otimes G(B) \rightarrow$ $G(A \otimes B)$ adjoint to

$$
F(G(A) \otimes G(B)) \stackrel{\sim}{\longrightarrow} F(G(A)) \otimes F(G(B)) \rightarrow A \otimes B .
$$

This construction extends to left-lax symmetric structures on $F$ and provides a bijection between left-lax symmetric structures on $F$ and right-lax symmetric structures on $G$. Since we do not need this extension, we omit the details.

Example A.1.7. Let $\mathscr{C}$ be a symmetric monoidal category. Then $\mathscr{C} \times \mathscr{C}$ is a symmetric monoidal category and the functor $-\otimes-: \mathscr{C} \times \mathscr{C} \rightarrow \mathscr{C}$ is a symmetric monoidal functor and, in particular, a symmetric functor. The symmetric structure of the functor is given by the isomorphisms $\left(A \otimes A^{\prime}\right) \otimes\left(B \otimes B^{\prime}\right) \stackrel{\sim}{\longrightarrow}(A \otimes B) \otimes\left(A^{\prime} \otimes B^{\prime}\right)$ for objects $A, A^{\prime}, B, B^{\prime}$ of $\mathscr{C}$.

Construction A.1.8. Let $\mathscr{C}$ and $\mathscr{D}$ be symmetric categories and let $G: \mathscr{C} \rightarrow \mathscr{D}$ be a right-lax symmetric functor. Let $A, B, K$ be objects of $\mathscr{C}$. A pairing $A \otimes B \rightarrow K$ induces a pairing $G(A) \otimes G(B) \rightarrow G(A \otimes B) \rightarrow G(K)$.

The following lemma follows immediately from the definitions.

Lemma A.1.9. Let $\mathscr{C}$ and $\mathscr{D}$ be symmetric categories and let $G: \mathscr{C} \rightarrow \mathscr{D}$ be a right-lax symmetric functor. Let $A, B, K$ be objects of $\mathscr{C}$. Let $A \otimes B \rightarrow K$ and $B \otimes A \rightarrow K$ be transposes of each other. Then the induced pairings $G A \otimes G B \rightarrow G K$ and $G B \otimes G A \rightarrow G K$ are transposes of each other.

A.2. Categories with duality. In this subsection, we study symmetry in categories with duality.

Definition A.2.1 (duality). Let $\mathscr{C}$ be a category. A duality on $\mathscr{C}$ is a functor $D: \mathscr{D}^{\mathrm{op}} \rightarrow \mathscr{D}$ endowed with a natural transformation ev $: \mathrm{id}_{\mathscr{C}} \rightarrow D D$ such that the composite $D \stackrel{\text { ev } D}{\longrightarrow} D D D \stackrel{D \text { ev }}{\longrightarrow} D$ is isomorphic to $\mathrm{id}_{D}$. The duality $(D$, ev) is said to be strong if ev is a natural isomorphism.

We are mostly interested in strong dualities in the main text. However, for the proofs of many results on strong dualities, it is necessary to consider general dualities (for example, the duality $D_{\mathrm{R} f_{*} K_{X}}$ in the proof of Remark 2.1.4(3) is not strong in general). Our terminology here is consistent with [Schlichting 2010, Definition 3.1]. Some authors refer to a strong duality simply as "duality" [Quebbemann et al. 1979]. 
The underlying functor of a strong duality is an equivalence of categories. If $\mathscr{C}$ is an additive category, we say that a duality on $\mathscr{C}$ is additive if the underlying functor is additive. By an additive category with duality, we mean an additive category equipped with an additive duality.

A basic example of duality is provided by the internal Hom functor in a closed symmetric category. We will discuss this in detail in Section A.4. By analogy with this case, we sometimes refer to morphisms $B \rightarrow D A$ in a category with duality as forms. We have the following notion of symmetry for forms.

Definition A.2.2 (symmetry of forms). Let ( $\mathscr{C}, D$, ev) be a category with duality for $\sigma=1$ (resp. additive category with duality for $\sigma=-1$ ) and let $A, B$ be objects of $\mathscr{D}$.

(1) We define the transpose of a morphism $g: B \rightarrow D A$ to be the composite

$$
A \stackrel{\mathrm{ev}}{\longrightarrow} D D A \stackrel{D g}{\longrightarrow} D B .
$$

We call $\sigma g^{T}$ the $\sigma$-transpose of $g$.

(2) We say that a morphism $f: A \rightarrow D A$ is $\sigma$-symmetric if $f=\sigma f^{T}$.

Again we will often say "symmetric" instead of "1-symmetric". The terminology above is justified by the following lemma.

Lemma A.2.3. We have $\left(g^{T}\right)^{T}=g$. The map $\operatorname{Hom}_{\mathscr{C}}(B, D A) \rightarrow \operatorname{Hom}_{\mathscr{C}}(A, D B)$ carrying $g$ to $g^{T}$ is a bijection.

Proof. The first assertion follows from the commutativity of the diagram

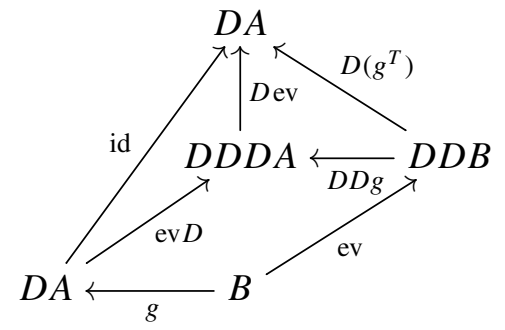

For the second assertion, note that the map carrying $h: A \rightarrow D B$ to $h^{T}$ is the inverse of the map $g \mapsto g^{T}$, by the first assertion.

Remark A.2.4. If $(\mathscr{C}, D$, ev $)$ is an additive category with duality, then $(\mathscr{C}, D,-\mathrm{ev})$ is another additive category with duality. The -1 -transpose in $(\mathscr{C}, D$, ev) of a morphism $g: B \rightarrow D A$ is the transpose in $(\mathscr{C}, D,-\mathrm{ev})$ of $g$. This allows us in the sequel to omit the -1 -symmetric case in many results without loss of generality.

We will be especially interested in objects $A$ that admit isomorphisms $A \stackrel{\sim}{\longrightarrow} D A$. Definition A.2.5. Let $(\mathscr{C}, D$, ev) be a category with duality and $A$ an object of $\mathscr{C}$.

(1) We say that $A$ is self-dual if there exists an isomorphism $A \stackrel{\sim}{\longrightarrow} D A$. 
(2) Assume that $(\mathscr{C}, D$, ev) is an additive category with duality if $\sigma=-1$. We say that $A$ is $\sigma$-self-dual if there exists a $\sigma$-symmetric isomorphism $A \stackrel{\sim}{\longrightarrow} D A$.

We warn the reader that being 1-self-dual is more restrictive than being self-dual. If $A$ is 1 -self-dual or -1 -self-dual, then ev : $A \rightarrow D D A$ is an isomorphism.

Remark A.2.6. Let $(\mathscr{C}, D$, ev) be an additive category with duality.

(1) The classes of self-dual objects and $\sigma$-self-dual objects of $\mathscr{D}$ are stable under finite products.

(2) If $A$ is an object of $\mathscr{D}$ such that ev : $A \rightarrow D D A$ is an isomorphism, then $A \oplus D A$ is 1-self-dual and -1 -self-dual. In fact, the isomorphism $A \oplus D A \stackrel{\sigma \text { ev} \oplus \mathrm{id}}{\longrightarrow}$ $D D A \oplus D A \simeq D(A \oplus D A)$ is $\sigma$-symmetric.

(3) Some self-dual objects are neither 1-self-dual nor -1-self-dual (Corollary 2.2.6).

We close this subsection with a couple of lemmas on $\sigma$-self-dual objects. They are used in Section 2.2 but not in the rest of this appendix.

A $\sigma$-symmetric isomorphism $f: A \stackrel{\sim}{\longrightarrow} D A$ induces an involution on $\operatorname{End}(A)$ carrying $g \in \operatorname{End}(A)$ to $f^{-1}(D g) f$. If $\mathscr{C}$ is a $k$-linear category and $D$ is a $k$-linear functor, then the involution is $k$-linear.

Lemma A.2.7. Let $(\mathscr{C}, D$, ev) be an additive category with duality. Let $A$ be an object of $\mathscr{D}$ such that $R=\operatorname{End}(A)$ is a local ring and 2 is invertible in $R$.

(1) If A is self-dual with respect to $D$, then $A$ is 1-self-dual or-1-self-dual with respect to $D$.

(2) If $A$ is both 1-self-dual and-1-self-dual with respect to $D$, then every symmetric (resp. -1-symmetric) isomorphism $f: A \rightarrow D A$ induces a nontrivial involution on the residue division ring of $R$.

This is essentially [Quebbemann et al. 1979, Proposition 2.5]. We recall the proof in our notation. It will be apparent from the proof that the additional assumption in [Quebbemann et al. 1979] that $D$ is a strong duality is not used.

Proof. (1) Since $A \simeq D A$, we have $\operatorname{End}(A) \simeq \operatorname{Hom}(A, D A)$. The image $M \subseteq$ $\operatorname{Hom}(A, D A)$ of the maximal ideal of $R=\operatorname{End}(A)$ is the complement of the set of isomorphisms. For any $f \in \operatorname{Hom}(A, D A)$, we have $2 f=\left(f+f^{T}\right)+\left(f-f^{T}\right)$, where $f+f^{T}$ is symmetric and $f-f^{T}$ is -1 -symmetric. If $f$ is an isomorphism, then $2 f$ is an isomorphism, so that either $f+f^{T}$ or $f-f^{T}$ is an isomorphism.

(2) Let $g: A \rightarrow D A$ be a -1 -symmetric (resp. symmetric) isomorphism. Then $h=f^{-1} g$ is a unit of $R$ whose image under the involution induced by $f$ is $-h$. Thus the involution is nontrivial on the residue field of $R$.

Remark A.2.8. (1) If $\mathscr{C}$ is an abelian category and $A$ is an indecomposable object of finite length, then End $(A)$ is a local ring [Atiyah 1956, Lemma 7]. 
(2) Let $k$ be a separably closed field of characteristic $\neq 2$. Assume that $\mathscr{C}$ is a $k$-linear category, $D$ is a $k$-linear functor, and $R=\operatorname{End}(A)$ is a finite $k$-algebra. Then any $k$-linear involution on $R$ is trivial on the residue field. It follows then from Lemma A.2.7 that exactly one of the following holds: $A$ is 1-self-dual; $A$ is -1 -self-dual; $A$ is not self-dual.

Lemma A.2.9. Let ( $D$, ev) be a strong duality on an abelian category $\mathscr{C}$. Let $A$ be a $\sigma$-self-dual object of finite length. Then the semisimplification $A^{\mathrm{ss}}$ of $A$ is $\sigma$-self-dual.

Note that by assumption $D$ is an equivalence of categories, hence an exact functor. Proof. We fix a $\sigma$-symmetric isomorphism $f: A \rightarrow D A$. For any subobject $N$ of $A$, we let $N^{\perp}$ denote the kernel of the morphism $A \stackrel{f}{\sim} D A \rightarrow D N$. Then we have $A / N^{\perp} \simeq D N$, so that $N^{\mathrm{ss}} \oplus\left(A / N^{\perp}\right)^{\mathrm{ss}}$ is $\sigma$-self-dual by Remark A.2.6. If $N$ is totally isotropic, namely $N \subseteq N^{\perp}$, then $f$ induces a $\sigma$-symmetric isomorphism $N^{\perp} / N \stackrel{\sim}{\longrightarrow} D\left(N^{\perp} / N\right)$ (see [Quebbemann et al. 1979, Lemma 5.2]). Now let $N$ be a maximal totally isotropic subobject of $A$. By [Quebbemann et al. 1979, Theorem 6.12], $N^{\perp} / N$ is semisimple. Therefore, $A^{\mathrm{ss}} \simeq N^{\mathrm{ss}} \oplus\left(A / N^{\perp}\right)^{\mathrm{ss}} \oplus N^{\perp} / N$ is $\sigma$-self-dual.

A.3. Duality and functors. In this subsection, we study symmetry of functors between categories with duality.

Given categories with duality $\left(\mathscr{C}, D_{\mathscr{C}}, \mathrm{ev}\right)$ and $\left(\mathscr{D}, D_{\mathscr{D}}, \mathrm{ev}\right)$, and functors $F, G$ : $\mathscr{C} \rightarrow \mathscr{D}$, we sometimes refer to natural transformations $G D_{\mathscr{C}} \rightarrow D_{\mathscr{D}} F$ as form transformations. If $\mathscr{C}$ is the category with one object $*$ and one morphism id, and if we identify functors $\{*\} \rightarrow \mathscr{D}$ with objects of $\mathscr{D}$, then a form transformation is simply a form in $\mathscr{D}$. Form transformations are composed as follows.

Construction A.3.1. Let $\left(\mathscr{B}, D_{\mathscr{B}}\right.$, ev $),\left(\mathscr{C}, D_{\mathscr{C}}, \mathrm{ev}\right)$, and $\left(\mathscr{D}, D_{\mathscr{D}}\right.$, ev) be categories with duality. Let $F, G: \mathscr{C} \rightarrow \mathscr{D}$ and $F^{\prime}, G^{\prime}: \mathscr{B} \rightarrow \mathscr{C}$ be functors. Let $\alpha: F D_{\mathscr{C}} \rightarrow D_{\mathscr{D}} G$ and $\alpha^{\prime}: F^{\prime} D_{\mathscr{B}} \rightarrow D_{\mathscr{C}} G^{\prime}$ be natural transformations. We define the composite of $\alpha$ and $\alpha^{\prime}$ to be

$$
\alpha \alpha^{\prime}: F F^{\prime} D_{\mathscr{B}} \stackrel{F \alpha^{\prime}}{\longrightarrow} F D_{\mathscr{C}} G^{\prime} \stackrel{\alpha G^{\prime}}{\longrightarrow} D_{\mathscr{D}} G G^{\prime}
$$

As the name suggests, form transformations act on forms. This can be seen as the case $\mathscr{B}=\{*\}$ of the preceding construction, as follows.

Construction A.3.2. Let $\left(\mathscr{C}, D_{\mathscr{C}}\right.$, ev) and $\left(\mathscr{D}, D_{\mathscr{D}}\right.$, ev) be categories with duality. Let $F, G: \mathscr{C} \rightarrow \mathscr{D}$ be functors and let $\alpha: F D_{\mathscr{C}} \rightarrow D_{\mathscr{D}} G$ be a natural transformation. Let $A, B$ be objects of $\mathscr{C}$ and let $f: A \rightarrow D_{\mathscr{C}} B$ be a morphism in $\mathscr{C}$. The action of $\alpha$ on $f$ is the composite

$$
\alpha f: F A \stackrel{F f}{\longrightarrow} F D_{\mathscr{C}} B \stackrel{\alpha_{B}}{\longrightarrow} D_{\mathscr{D}} G B .
$$


We have the following notion of symmetry for form transformations.

Definition A.3.3 (symmetry of form transformations). Let $\left(\mathscr{C}, D_{\mathscr{C}}, \mathrm{ev}\right),\left(\mathscr{D}, D_{\mathscr{D}}\right.$, ev) be categories with duality. Let $F, G: \mathscr{C} \rightarrow \mathscr{D}$ be functors. Assume that $\left(\mathscr{D}, D_{\mathscr{D}}\right.$, ev) is an additive category with duality if $\sigma=-1$.

(1) We define the transpose of a natural transformation $\beta: G D_{\mathscr{C}} \rightarrow D_{\mathscr{D}} F$ to be the composite

$$
\beta^{T}: F D_{\mathscr{C}} \stackrel{\text { ev } F D_{\mathscr{C}}}{\longrightarrow} D_{\mathscr{D}} D_{\mathscr{D}} F D_{\mathscr{C}} \stackrel{D_{\mathscr{D}} \beta D_{\mathscr{C}}}{\longrightarrow} D_{\mathscr{D}} G D_{\mathscr{C}} D_{\mathscr{C}} \stackrel{D_{\mathscr{D}} G \mathrm{ev}}{\longrightarrow} D_{\mathscr{D}} G .
$$

We call $\sigma \beta^{T}$ the $\sigma$-transpose of $\beta$.

(2) We say that a natural transformation $\alpha: F D_{\mathscr{C}} \rightarrow D_{\mathscr{D}} F$ is $\sigma$-symmetric if $\alpha=\sigma \alpha^{T}$.

Again we will often say "symmetric" instead of "1-symmetric". The terminology above is justified by the following easy lemma.

Lemma A.3.4. We have $\left(\beta^{T}\right)^{T}=\beta$. The map $\operatorname{Nat}\left(G D_{\mathscr{C}}, D_{\mathscr{D}} F\right) \rightarrow \operatorname{Nat}\left(F D_{\mathscr{C}}, D_{\mathscr{D}} G\right)$ carrying $\beta$ to $\beta^{T}$ is a bijection.

The transpose $\alpha=\beta^{T}$ is uniquely characterized by the commutativity of the diagram

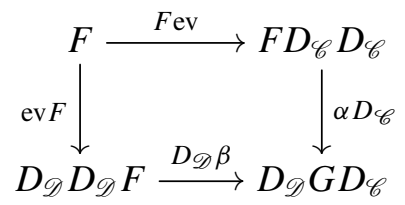

If $\mathscr{C}=\{*\}$, Definition A.3.3 reduces to Definition A.2.2.

Remark A.3.5. A more direct analogue of Definition A.2.2(1) for functors is as follows. Let $\mathscr{C}$ be a category and let $\left(\mathscr{D}, D_{\mathscr{D}}\right.$, ev) be a category with duality. Let $G: \mathscr{C} \rightarrow \mathscr{D}$ and $H: \mathscr{C} \rightarrow \mathscr{D}^{\text {op }}$ be functors. Then the map $\operatorname{Nat}\left(G, D_{\mathscr{D}} H\right) \rightarrow$ $\operatorname{Nat}\left(H, D_{\mathscr{D}} G\right)$ carrying $\gamma: G \rightarrow D_{\mathscr{D}} H$ to $\gamma^{*}: H \stackrel{\text { ev } H}{\longrightarrow} D_{\mathscr{D}} D_{\mathscr{D}} H \stackrel{D_{\mathscr{D}}}{\longrightarrow} D_{\mathscr{D}} G$ is a bijection. Indeed, $\gamma$ is a collection $\left(\gamma_{A}: G A \rightarrow D_{\mathscr{D}} H A\right)_{A}$ of forms in $\mathscr{D}$ and $\gamma^{*}$ is characterized by $\left(\gamma^{*}\right)_{A}=\left(\gamma_{A}\right)^{T}$ for all objects $A$ of $\mathscr{C}$, so that $\left(\gamma^{*}\right)^{*}=\gamma$. As one of the referee points out, this operation does not lead to a notion of symmetry, since $G$ and $H$ do not have the same variance.

Remark A.3.6. In the situation of Definition A.3.3, we have a bijection

$$
\operatorname{Nat}\left(G D_{\mathscr{C}}, D_{\mathscr{D}} F\right) \stackrel{\sim}{\longrightarrow} \operatorname{Nat}\left(F, D_{\mathscr{D}} G D_{\mathscr{C}}\right)
$$

carrying $\beta$ to $\beta^{*}$. Note that $\beta^{*}$ is the composite $F \stackrel{F \mathrm{ev}}{\longrightarrow} F D_{\mathscr{C}} D_{\mathscr{C}} \stackrel{\beta^{T} D_{\mathscr{C}}}{\longrightarrow} D_{\mathscr{D}} G D_{\mathscr{C}}$, and $\beta^{T}$ is the composite $F D_{\mathscr{C}} \stackrel{\beta^{*} D_{\mathscr{C}}}{\longrightarrow} D_{\mathscr{D}} G D_{\mathscr{C}} D_{\mathscr{C}} \stackrel{D_{\mathscr{D}} G \mathrm{ev}}{\longrightarrow} D_{\mathscr{D}} G$.

If we equip the functor category $\operatorname{Fun}(\mathscr{C}, \mathscr{D})$ with the duality carrying $G$ to $D_{\mathscr{D}} G D_{\mathscr{C}}$ and the evaluation transformation given by $G \stackrel{\mathrm{ev} G \mathrm{ev}}{\longrightarrow} D_{\mathscr{D}} D_{\mathscr{D}} G D_{\mathscr{C}} D_{\mathscr{C}}$, then natural transformations $F \rightarrow D_{\mathscr{D}} G D_{\mathscr{C}}$ are forms in this category with duality, and 
Definition A.3.3 of transposes of forms applies. Definition A.3.3 is compatible with Definition A.2.2 via the bijection (A-3-2) in the sense that we have $\left(\beta^{*}\right)^{T}=\left(\beta^{T}\right)^{*}$.

Composition of form transformations is compatible with transposition.

Lemma A.3.7. Let $\left(\mathscr{B}, D_{\mathscr{B}}\right.$, ev), $\left(\mathscr{C}, D_{\mathscr{C}}, \mathrm{ev}\right),\left(\mathscr{D}, D_{\mathscr{D}}\right.$, ev) be categories with duality. Let $F, G: \mathscr{C} \rightarrow \mathscr{D}$ and $F^{\prime}, G^{\prime}: \mathscr{B} \rightarrow \mathscr{C}$ be functors. Let $\alpha: F D_{\mathscr{C}} \rightarrow D_{\mathscr{D}} G$ and $\alpha^{\prime}: F^{\prime} D_{\mathscr{B}} \rightarrow D_{\mathscr{C}} G^{\prime}$ be natural transformations and let $\alpha \alpha^{\prime}: F F^{\prime} D_{\mathscr{B}} \rightarrow D_{\mathscr{D}} G G^{\prime}$ be the composite. Then $\left(\alpha \alpha^{\prime}\right)^{T}=\alpha^{T} \alpha^{\prime T}$.

Proof. In the diagram

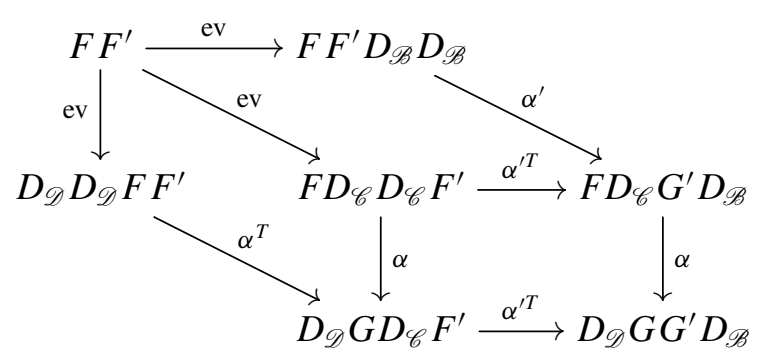

all inner cells commute. It follows that the outer hexagon commutes.

Taking $\mathscr{B}=\{*\}$, we obtain the following compatibility of transposition with the action of form transformations.

Lemma A.3.8. Let $\left(\mathscr{C}, D_{\mathscr{C}}\right.$, ev) and $\left(\mathscr{D}, D_{\mathscr{D}}\right.$, ev) be categories with duality. Let $F, G: \mathscr{C} \rightarrow \mathscr{D}$ be functors equipped with a natural transformation $\alpha: F D_{\mathscr{C}} \rightarrow D_{\mathscr{D}} G$. Let $f: A \rightarrow D_{\mathscr{C}} B$ be a morphism in $\mathscr{C}$. Then $(\alpha f)^{T}=\alpha^{T} f^{T}$.

The following consequence of Lemma A.3.8 is used many times in Section 2.

Lemma A.3.9. Let $\left(\mathscr{C}, D_{\mathscr{C}}\right.$, ev) and $\left(\mathscr{D}, D_{\mathscr{D}}\right.$, ev) be categories with duality, and let $F: \mathscr{C} \rightarrow \mathscr{D}$ be a functor endowed with a symmetric natural isomorphism $\alpha: F D_{\mathscr{C}} \stackrel{\sim}{\longrightarrow} D_{\mathscr{D}} F$.

(1) F carries 1-self-dual objects of $\mathscr{C}$ to 1-self-dual objects of $\mathscr{D}$.

(2) If $F$ is fully faithful, then the converse holds: any object $A$ of $\mathscr{C}$ such that FA is 1-self-dual is 1-self-dual.

Proof. For (1), let $f: A \stackrel{\sim}{\longrightarrow} D_{\mathscr{C}} A$ be a symmetric isomorphism. By Lemma A.3.8, $\alpha f$ is symmetric. The assertion follows from the fact that $\alpha f$ is an isomorphism. For (2), let $g: F A \stackrel{\sim}{\longrightarrow} D_{\mathscr{D}} F A$ be a symmetric isomorphism. Since $F$ is fully faithful, there exists a unique morphism $f: A \rightarrow D_{\mathscr{C}} A$ such that $\alpha f=g$. Note that $f$ is an isomorphism. Since $\alpha f^{T}=g$, we have $f^{T}=f$.

The following lemma is used in Section 2.1 to show the symmetry of the middle extension functor. 
Lemma A.3.10. Let $\left(\mathscr{C}, D_{\mathscr{C}}, \mathrm{ev}\right)$ and $\left(\mathscr{D}, D_{\mathscr{D}}, \mathrm{ev}\right)$ be categories with duality. Assume that $\mathscr{D}$ is an abelian category and that $D_{\mathscr{D}}$ carries epimorphisms in $\mathscr{D}$ to monomorphisms. Let $E, G: \mathscr{C} \rightarrow \mathscr{D}$ be functors endowed with natural transformations $\alpha: E \rightarrow G$ and $\beta: G D_{\mathscr{C}} \rightarrow D_{\mathscr{D}} E$ such that the composite $E D_{\mathscr{C}} \stackrel{\alpha D_{\mathscr{C}}}{\longrightarrow}$ $G D_{\mathscr{C}} \stackrel{\beta}{\longrightarrow} D_{\mathscr{D}} E$ is symmetric and such that the image functor $F: \mathscr{C} \rightarrow \mathscr{D}$ of $\alpha$ fits into a commutative diagram

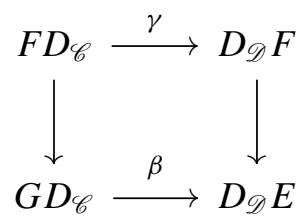

Then the natural transformation $\gamma: F D_{\mathscr{C}} \rightarrow D_{\mathscr{D}} F$ is symmetric.

Proof. In fact, in the diagram

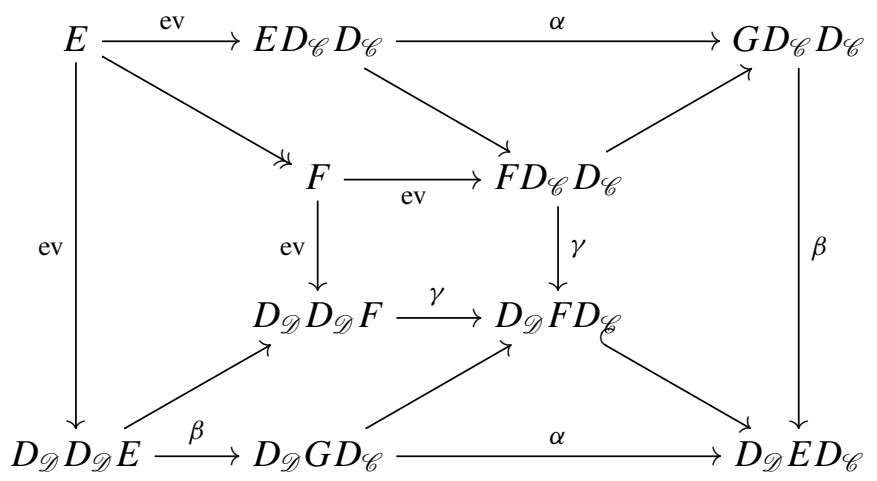

the outer square commutes by the symmetry of $\beta \alpha$ and all inner cells except the inner square commute. It follows that the inner square commutes.

We conclude this subsection with another example of form transformation, which will be used to handle the sign of the Lefschetz pairing (see Lemma A.5.11). We refer to [Kashiwara and Schapira 2006, Remark 10.1.10(ii)] for the convention on distinguished triangles in the opposite category of a triangulated category.

Lemma A.3.11. Let $\mathscr{D}$ be a triangulated category equipped with a $t$-structure $P$. Let $(D, \mathrm{ev}): \mathscr{D}^{\mathrm{op}} \rightarrow \mathscr{D}$ be a duality on the underlying category of $\mathscr{D}$. Assume that $D$ underlies a right t-exact triangulated functor. We consider $\tau={ }^{P_{\tau} \geq a}$ and $\tau^{\prime}={ }^{P} \tau^{\leq-a}$ as functors $\mathscr{D} \rightarrow \mathscr{D}$. Then the form transformations $\tau D \rightarrow D \tau^{\prime}$ and $\tau^{\prime} D \rightarrow D \tau$ induced by the diagrams

$$
\begin{aligned}
& \tau D \rightarrow \tau D \tau^{\prime} \stackrel{\sim}{\longleftarrow} \tau^{\prime}, \\
& \tau^{\prime} D \longleftarrow \tau^{\prime} D \tau \rightarrow D \tau
\end{aligned}
$$

are transposes of each other. 
The second arrow in (A-3-3) is an isomorphism by the assumption that $D$ carries ${ }^{P}{ }_{\tau} \leq-a$ to ${ }^{P_{\tau} \geq-a}$. To see that the first arrow in (A-3-4) is an isomorphism, consider, for any object $A$ of $\mathscr{D}$, the distinguished triangle

$$
D^{P} \tau^{\geq a} A \stackrel{f}{\longrightarrow} D A \rightarrow D^{P} \tau^{\leq a-1} A \rightarrow .
$$

By assumption, $D^{P \leq a-1} A$ is in ${ }^{P} \mathscr{D}^{\geq 1-a}$. Thus, by Lemma $4.1 .9,{ }^{P_{\tau} \leq-a} f$ is an isomorphism.

Proof. The commutativity of (A-3-1) follows from the commutativity of the diagram

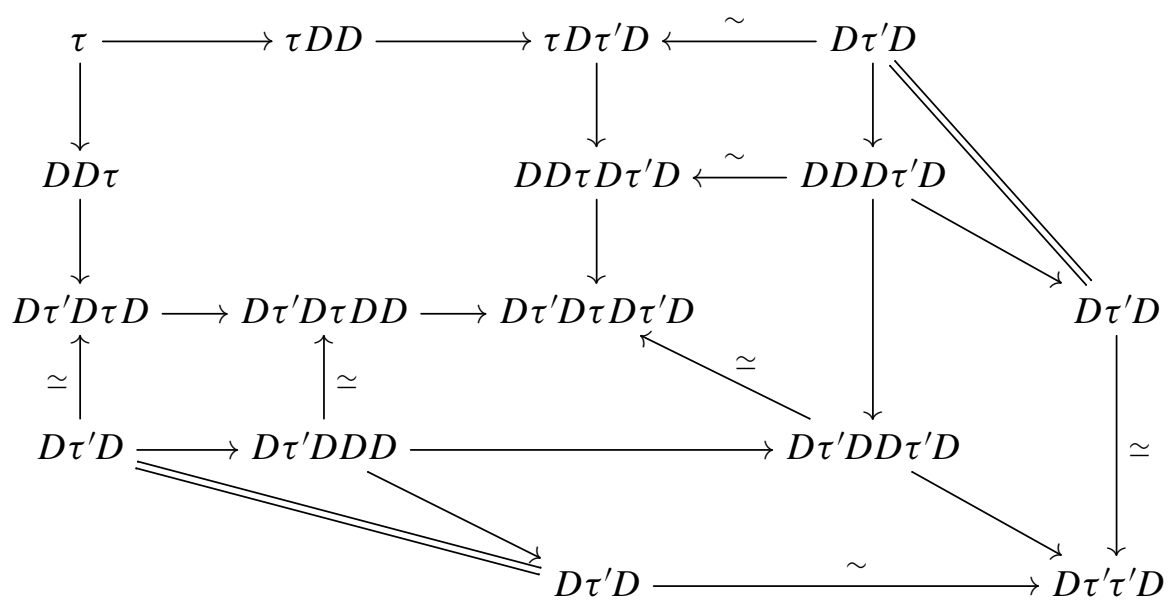

Remark A.3.12. For any truncation functor $\tau={ }^{P} \tau^{[a, b]}$ with dual truncation functor $\tau^{\prime}={ }^{P} \tau^{[-b,-a]}$, combining the two form transformations in the lemma, we obtain a form transformation $\gamma_{\tau}: \tau D \rightarrow D \tau^{\prime}$ whose transpose is $\gamma_{\tau}^{\prime}$. The form transformation $\gamma_{\tau}$ is an isomorphism if $D$ is $t$-exact.

A.4. Duality in closed symmetric categories. In this subsection, we study dualities given by internal Hom functors in closed symmetric categories. Let $(\mathscr{C}, \otimes, c)$ be a closed symmetric category (Definition A.1.1).

Construction A.4.1. Let $K$ be an object of $\mathscr{C}$, and let $D_{K}$ denote the functor $\mathscr{H}$ om $(-, K): \mathscr{D}^{\text {op }} \rightarrow \mathscr{D}$. For an object $A$, the composite

$$
A \otimes D_{K} A \stackrel{c}{\longrightarrow} D_{K} A \otimes A \stackrel{\text { adj }}{\longrightarrow} K,
$$

where adj denotes the adjunction morphism, corresponds by adjunction to a morphism $A \rightarrow D_{K} D_{K} A$. This defines a natural transformation ev : $\mathrm{id}_{\mathscr{D}} \rightarrow D_{K} D_{K}$, which makes $D_{K}$ a duality on $\mathscr{D}$. The latter follows by adjunction from the commutativity 
of the diagram

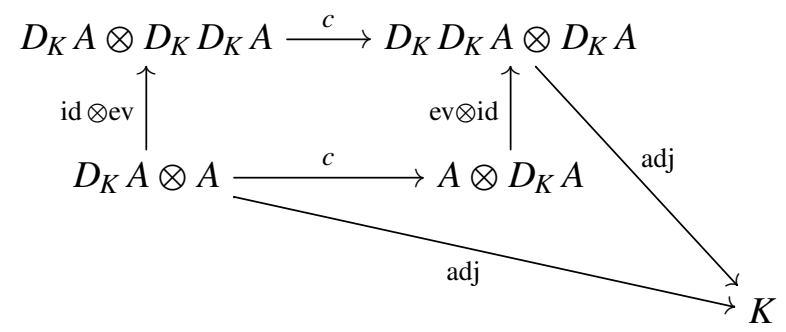

We defined transposes of pairings in symmetric categories (Definition A.2.2) and in categories with duality (Definition A.1.3). The two definitions are compatible via the above construction, by the following lemma.

Lemma A.4.2. Let $A, B, K$ be objects of $\mathscr{C}$, and set $D=D_{K}$. Then the following diagram commutes:

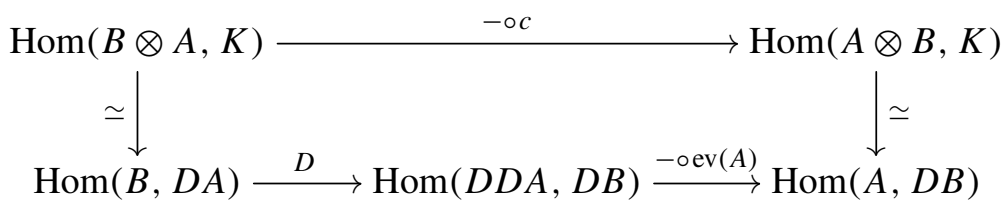

Proof. Let $f \in \operatorname{Hom}(B, D A)$. The two images of $f$ in $\operatorname{Hom}(A \otimes B, K)$ are the two composite morphisms in the commutative diagram

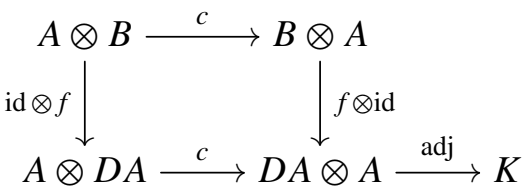

Following Definition A.2.5, we say $A$ is self-dual with respect to $K$ if $A \simeq D_{K} A$. We say $A$ is $\sigma$-self-dual with respect to $K$ if there exists a $\sigma$-symmetric isomorphism $A \stackrel{\sim}{\longrightarrow} D_{K} A$, or, in other words, if there exists a $\sigma$-symmetric pairing $A \otimes A \rightarrow K$ that is perfect in the sense that it induces an isomorphism $A \stackrel{\sim}{\longrightarrow} D_{K} A$.

Definition A.4.3. A dualizing object of $\mathscr{C}$ is an object $K$ of $\mathscr{C}$ such that the evaluation transformation ev $: \operatorname{id}_{\mathscr{C}} \rightarrow D_{K} D_{K}$ is a natural isomorphism, or, in other words, such that $\left(D_{K}, \mathrm{ev}\right)$ is a strong duality.

Remark A.4.4. Let $\mathscr{B}$ be a closed symmetric monoidal category and let $K$ be an object of $\mathscr{B}$. The associativity constraint induces an isomorphism $\mathscr{H} o m\left(A, D_{K} B\right) \simeq$ $D_{K}(A \otimes B)$ for objects $A, B$ of $\mathscr{D}$. In particular, if $K$ is a dualizing object, then $\mathscr{H} o m(A, B) \simeq \mathscr{H} o m\left(A, D_{K} D_{K} B\right) \simeq D_{K}\left(A \otimes D_{K} B\right)$. 
We close this subsection by constructing two symmetric form transformations.

Construction A.4.5. For a morphism $f: K \rightarrow L$ of $\mathscr{C}$, the natural transformation $D_{f}: \operatorname{id}_{\mathscr{C}} D_{K} \rightarrow D_{L} \mathrm{id}_{\mathscr{C}}$ is symmetric. This follows from the commutativity of the diagram

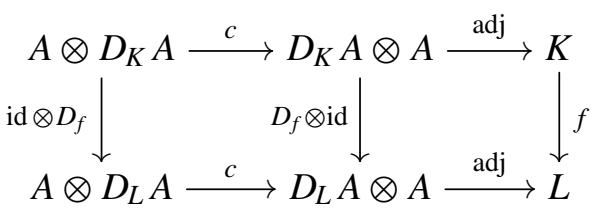

The action of $D_{f}$ on forms (Construction A.3.2) carries $A \otimes B \rightarrow K$ to the composite $A \otimes B \rightarrow K \stackrel{f}{\longrightarrow} L$.

Construction A.4.6. Let $\mathscr{C}$ and $\mathscr{D}$ be closed symmetric categories and let $G: \mathscr{C} \rightarrow \mathscr{D}$ be a right-lax symmetric functor (Definition A.1.5). For objects $A, K$ of $\mathscr{C}$, consider the morphism

$$
\begin{aligned}
G \mathscr{H} o m(A, K) \stackrel{\text { adj }}{\longrightarrow} & \mathscr{H o m}(G A, G \mathscr{H o m}(A, K) \otimes G A) \\
\rightarrow & \mathscr{H} o m(G A, G(\mathscr{H} o m(A, K) \otimes A)) \stackrel{\text { adj }}{\longrightarrow} \mathscr{H} o m(G A, G K) .
\end{aligned}
$$

This induces a symmetric natural transformation $G D_{K} \rightarrow D_{G K} G$ (see [Riou 2014, Théorème 12.2.5]), whose action on forms carries $A \otimes B \rightarrow K$ to the pairing $G A \otimes G B \rightarrow G K$ of Construction A.1.8.

A.5. Symmetry and translation. The derived category of $\ell$-adic sheaves is equipped with a shift functor $A \mapsto A[1]$ and the Tate twist functor $A \mapsto A(1)$. In this subsection, we study the effects of such translation functors on symmetry. Lemma A.5.11 is used in the main text to handle the symmetry of the Lefschetz pairing.

Recall that a category with translation [Kashiwara and Schapira 2006, Definition 10.1.1(i)] is a category $\mathscr{D}$ equipped with an equivalence of categories $T: \mathscr{D} \rightarrow \mathscr{D}$. We let $T^{-1}: \mathscr{D} \rightarrow \mathscr{D}$ denote a quasi-inverse of $T$. For an integer $n$, we will often write $[n]$ for $T^{n}$. Recall that a functor of categories of translation [Kashiwara and Schapira 2006, Definition 10.1.1(ii)] $(\mathscr{D}, T) \rightarrow\left(\mathscr{D}^{\prime}, T^{\prime}\right)$ is a functor $F: \mathscr{D} \rightarrow \mathscr{D}^{\prime}$ endowed with a natural isomorphism $\eta: F T \stackrel{\sim}{\longrightarrow} T^{\prime} F$. Recall that a morphism of functors of categories with translation $(F, \eta) \rightarrow(G, \xi)$ is a natural transformation $\alpha: F \rightarrow G$ of functors such that the following diagram commutes:

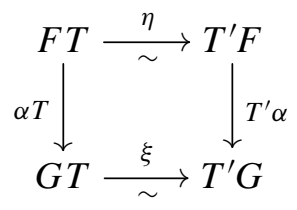


Our first goal is to define duality on categories with translation, a variant of Definition A.2.1. We endow $\mathscr{D}^{\mathrm{op}}$ with the translation functor $\left(T^{\mathrm{op}}\right)^{-1}: \mathscr{D}^{\mathrm{op}} \rightarrow \mathscr{D}^{\mathrm{op}}$. We endow $F^{\text {op }}: \mathscr{A}^{\text {op }} \rightarrow \mathscr{A}^{\text {op }}$ with the isomorphism $F^{\text {op }}\left(T^{\text {op }}\right)^{-1} \stackrel{\sim}{\longrightarrow}\left(T^{\text {op }}\right)^{-1} F^{\text {op }}$ induced by

$$
\eta^{\mathrm{op}}: T^{\mathrm{op}} F^{\mathrm{op}} \stackrel{\sim}{\longrightarrow} F^{\mathrm{op}} T^{\mathrm{op}} .
$$

Definition A.5.1 (duality on a category with translation). Let $(\mathscr{D}, T)$ be a category with translation. A duality on $(\mathscr{D}, T)$ is a functor of categories with translation $(D, \eta):\left(\mathscr{D}^{\mathrm{op}},\left(T^{\mathrm{op}}\right)^{-1}\right) \rightarrow(\mathscr{D}, T)$ endowed with a structure of duality on the underlying functor $D: \mathscr{D}^{\text {op }} \rightarrow \mathscr{D}$ such that $\mathrm{ev}: \mathrm{id}_{\mathscr{D}} \rightarrow D D^{\text {op }}$ is a morphism of functors of categories with translation. This means that the diagram

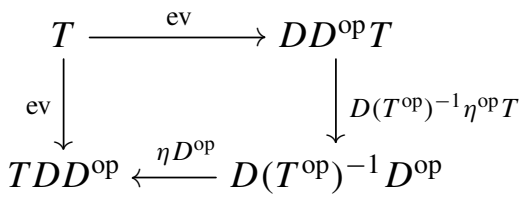

commutes. In other words, the isomorphisms $\eta^{-1}: T D \stackrel{\sim}{\longrightarrow} D\left(T^{\mathrm{op}}\right)^{-1}$ and $T^{-1} \eta T^{\mathrm{op}}$ : $T^{-1} D \stackrel{\sim}{\longrightarrow} D T^{\text {op }}$ are transposes of each other in the sense of Definition A.3.3.

The above definitions have obvious additive variants. An additive category with translation is defined to be a category with translation whose underlying category is additive. For additive categories with translation $\mathscr{D}$ and $\mathscr{D}^{\prime}$, a functor of additive categories with translation $\mathscr{D} \rightarrow \mathscr{D}^{\prime}$ is defined to be a functor of categories with translation whose underlying functor is additive. An additive duality on an additive category with translation is a duality on the category with translation such that the underlying functor is additive.

As in the case without translation, a basic example of dualities on categories with translation is provided by closed symmetric categories with translation (see Construction A.5.9 below). Our next goal is to define symmetric categories with translation, a variant of Definition A.1.1. Note that in the example of $\ell$-adic sheaves, the shift and twist functors differ in signs with regard to tensor products. To deal with the two cases simultaneously, we let $\epsilon= \pm 1$. The case $\epsilon=-1$ of the following definition corresponds to [Kashiwara and Schapira 2006, Definition 10.1.1(v)]. For a more general notion, see [Verdier 1996, Définition I.1.4.4].

Definition A.5.2. Let $\mathscr{D}, \mathscr{D}^{\prime}, \mathscr{D}^{\prime \prime}$ be additive categories with translation. An $\epsilon$-bifunctor of additive categories with translation $F: \mathscr{D} \times \mathscr{D}^{\prime} \rightarrow \mathscr{D}^{\prime \prime}$ is an additive bifunctor endowed with functorial isomorphisms $F(A[1], B) \simeq F(A, B)[1]$ and $F(A, B[1]) \simeq F(A, B)[1]$ for objects $A$ of $\mathscr{D}$ and $B$ of $\mathscr{D}^{\prime}$, such that the following 
diagram $\epsilon$-commutes:

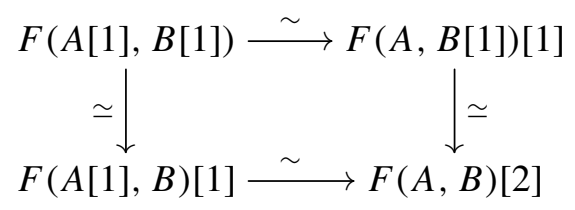

Therefore, the following diagram $\epsilon^{m n}$-commutes:

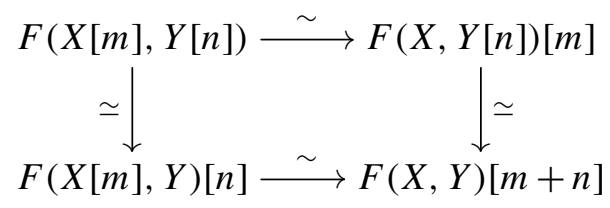

Definition A.5.3 (symmetric category with translation). An $\epsilon$-symmetric additive category with translation is an additive category with translation $\mathscr{D}$ endowed with a symmetric structure $\otimes$ and a structure of an additive $\epsilon$-bifunctor of categories with translation on $-\otimes-: \mathscr{D} \times \mathscr{D} \rightarrow \mathscr{D}$, such that the symmetry constraint, when restricted to each variable, is a morphism of functors of categories with translation $\mathscr{D} \rightarrow \mathscr{D}$. We say that an $\epsilon$-symmetric additive category with translation is closed if its underlying symmetric category is closed.

For $\epsilon=1$, Definitions A.5.2 and A.5.3 make sense without assuming that the categories in question are additive.

Example A.5.4. Let $\mathscr{C}$ be a symmetric monoidal category and let $X$ be a dualizable object of $\mathscr{C}$, that is, there exists an object $B$ of $\mathscr{C}$ such that $A \otimes B \simeq \mathbf{1}$. Then $-\otimes A$ endows $\mathscr{C}$ with the structure of a 1 -symmetric category with translation. This applies in particular to the Tate twist functor on the abelian category of perverse $\overline{\mathbb{Q}}_{\ell}$-sheaves.

Example A.5.5. The derived category of any commutatively ringed topos is a closed -1 -symmetric additive category with translation. Similarly, the derived category of $\overline{\mathbb{Q}}_{\ell}$-sheaves is a closed -1-symmetric additive category with translation.

Let $\mathscr{D}$ be an $\epsilon$-symmetric additive category with translation.

Lemma A.5.6. The diagram

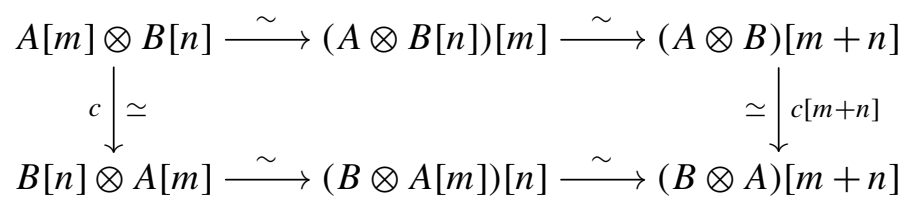

$\epsilon^{m n}$-commutes for all objects $A, B$ of $\mathscr{D}$ and all integers $m, n$. Here c denotes the symmetry constraint. 
Proof. In the diagram

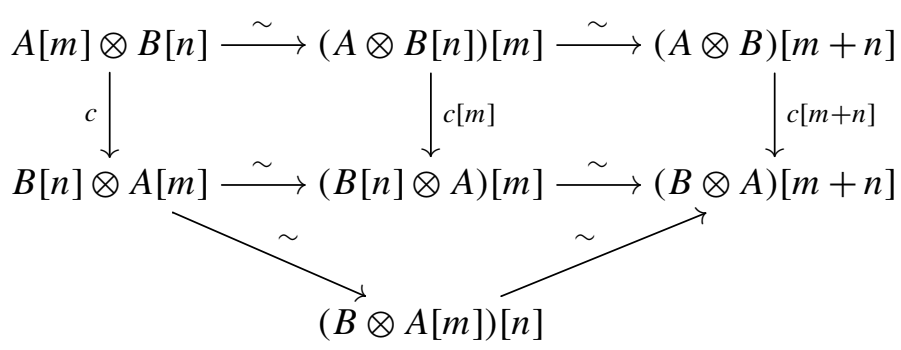

the upper squares commute by functoriality, and the lower triangle $\epsilon^{m n}$-commutes by the definition of $\epsilon$-bifunctor of additive categories with translation.

Construction A.5.7. Let $A, B, K$ be objects of $\mathscr{D}$. A pairing $A \otimes B \rightarrow K$ induces a pairing

$$
(A[m]) \otimes(B[n]) \simeq(A \otimes B[n])[m] \simeq(A \otimes B)[m+n] \rightarrow K[m+n] .
$$

Lemma A.5.6 implies the following.

Lemma A.5.8. Let $A, B, K$ be objects of $\mathscr{D}$. Let $A \otimes B \rightarrow K$ and $B \otimes A \rightarrow K$ be two pairings that are $\sigma$-transposes of each other. Then the induced pairings $(A[m]) \otimes(B[n]) \rightarrow K[m+n]$ and $(B[n]) \otimes(A[m]) \rightarrow K[m+n]$ are $\epsilon^{m n} \sigma$-transposes of each other.

Let $\mathscr{D}$ be a closed $\epsilon$-symmetric additive category with translation.

Construction A.5.9. Consider the isomorphisms

$$
\begin{aligned}
& \alpha_{n}: \mathscr{H o m}(A[-n], B) \stackrel{\sim}{\longrightarrow} \mathscr{H o m}(A, B)[n], \\
& \beta_{n}: \mathscr{H o m}(A, B[n]) \stackrel{\sim}{\longrightarrow} \mathscr{H o m}(A, B)[n],
\end{aligned}
$$

given by the isomorphisms

$\operatorname{Hom}(C, \mathscr{H o m}(A[-n], B)) \simeq \operatorname{Hom}(C \otimes A[-n], B) \simeq \operatorname{Hom}((C \otimes A)[-n], B)$,

$\operatorname{Hom}(C, \mathscr{H} o m(A, B[n])) \simeq \operatorname{Hom}(C \otimes A, B[n]) \simeq \operatorname{Hom}((C \otimes A)[-n], B)$, and

$\operatorname{Hom}((C \otimes A)[-n], B) \simeq \operatorname{Hom}(C[-n] \otimes A, B)$

$$
\simeq \operatorname{Hom}(C[-n], \mathscr{H} o m(A, B)) \simeq \operatorname{Hom}(C, \mathscr{H} o m(A, B)[n])
$$

for objects $A, B, C$ of $\mathscr{D}$. We have $\alpha_{m} \alpha_{n}=\epsilon^{m n} \alpha_{m+n}, \beta_{m} \beta_{n}=\beta_{m n}, \alpha_{m} \beta_{n}=\epsilon^{m n} \beta_{n} \alpha_{m}$. We endow $\mathscr{H} o m(-,-): \mathscr{D}^{\mathrm{op}} \times \mathscr{D} \rightarrow \mathscr{D}$ with the structure of an $\epsilon$-bifunctor of additive categories with translation given by $\epsilon \alpha_{1}$ and $\beta_{1}{ }^{3}$ Let $\tilde{\alpha}_{n}=\left(\epsilon \alpha_{1}\right)^{n}=\epsilon^{n(n+1) / 2} \alpha_{n}$.

${ }^{3}$ The sign convention is adopted only for concreteness. Our results do not depend on the convention. 
In particular, $D_{A}: \mathscr{D}^{\text {op }} \rightarrow \mathscr{D}$ is endowed with the structure of a functor of additive categories with translation, which, together with ev : $\operatorname{id}_{\mathscr{D}} \rightarrow D_{A} D_{A}^{\text {op }}$, defines an additive duality on the additive category with translation (see [Calmès and Hornbostel 2009, Proposition 3.2.1]).

Remark A.5.10. Construction A.5.7 corresponds to the construction that sends $f: A \rightarrow D_{K} B$ to $\epsilon^{n(n-1) / 2}$ times the morphism

$$
A[m] \stackrel{f[m]}{\longrightarrow}\left(D_{K} B\right)[m] \stackrel{\tilde{\alpha}_{-n}}{\sim} D_{K}(B[n])[m+n] \stackrel{\beta_{m+n}^{-1}}{\longrightarrow} D_{K[m+n]}(B[n]),
$$

where $\tilde{\alpha}$ and $\beta$ are as in Construction A.5.9. In fact, the following diagram $\epsilon^{n(n-1) / 2}$ commutes:

$\operatorname{Hom}(A[m] \otimes B[n], K[m+n]) \stackrel{\sim}{\rightarrow} \operatorname{Hom}((A \otimes B[n])[m], K[m+n])$

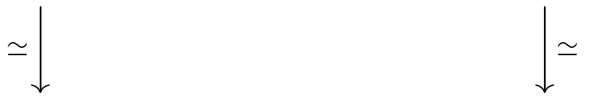

$\operatorname{Hom}\left(A[m], D_{K[m+n]}(B[n])\right) \quad \operatorname{Hom}((A \otimes B)[m+n], K[m+n]) \stackrel{\sim}{\rightarrow} \operatorname{Hom}(A \otimes B, K)$

$$
\simeq \downarrow \tilde{\alpha}_{-n}
$$

$\operatorname{Hom}\left(A[m], D_{K}(B[n])[m+n]\right) \stackrel{\beta_{m+n}^{-1}}{\sim} \operatorname{Hom}\left(A[m], D_{K} B[m]\right) \longrightarrow \operatorname{Hom}\left(A, D_{K} B\right)$

Thus, Construction A.5.7 corresponds to the form transformation $\gamma_{m, n}: T^{m} D_{K} \stackrel{\sim}{\longrightarrow}$ $D_{K[m+n]} T^{n}$, defined to be $\epsilon^{n(n-1) / 2}$ times the isomorphism

$$
T^{m} D_{K} \stackrel{\tilde{\alpha}_{-n}}{\sim} T^{m+n} D_{K} T^{n} \stackrel{\beta_{m+n}^{-1}}{\sim} D_{K[m+n]} T^{n}
$$

given by Construction A.5.9. By the above, the $\epsilon^{m n}$-transpose of $\gamma_{m, n}$ is $\gamma_{n, m}$.

We combine the above discussion on translation with our previous discussion on truncation into the following lemma, which is applied in the proof of Proposition 3.2.12 to the Lefschetz pairing.

Lemma A.5.11. Let $\mathscr{D}$ be a closed-1-symmetric additive category with translation. Assume that the underlying category with translation is further equipped with a triangulated structure and a $t$-structure $P$. Let $K$ and $L$ be objects of $\mathscr{D}$ such that $D_{L}$ is a right $t$-exact triangulated functor. For any $\sigma$-symmetric pairing $A \otimes A \rightarrow K$ and any morphism $\xi: K[2 n] \rightarrow L$, the pairing ${ }^{P} \mathrm{H}^{n} A \otimes{ }^{P} \mathrm{H}^{n} A \rightarrow L$ induced by

$$
A[n] \otimes A[n] \stackrel{\sim}{\longrightarrow}(A \otimes A)[2 n] \rightarrow K[2 n] \stackrel{\xi}{\longrightarrow} L
$$

is $(-1)^{n} \sigma$-symmetric.

In fact, the form transformation ${ }^{P} \mathrm{H}^{n} D_{K} \rightarrow D_{L}{ }^{P} \mathrm{H}^{n}$ given by

$$
\tau^{[0,0]} T^{n} D_{K} \stackrel{\gamma_{n, n}}{\longrightarrow} \tau^{[0,0]} D_{K[2 n]} T^{n} \stackrel{D_{\xi}}{\longrightarrow} \tau^{[0,0]} D_{L} T^{n} \stackrel{\gamma_{\tau}}{\longrightarrow} D_{L} \tau^{[0,0]} T^{n}
$$

is $(-1)^{n}$-symmetric. Here $\gamma_{n, n}$ and $\gamma_{\tau}$ are as in Remarks A.5.10 and A.3.12. 
Remark A.5.12. Let us mention in passing that Lurie's theory [2014, Chapter 1] of stable $\infty$-categories provides a nicer framework for symmetric monoidal structures in derived categories. If $(\mathscr{D}, \otimes)$ is a closed symmetric monoidal $\infty$-category such that the underlying $\infty$-category $\mathscr{D}$ is stable, then $-\otimes-$ and $\mathscr{H} o m(-,-)$ are automatically exact in each variable and the homotopy category of $\mathscr{D}$ is a closed -1 -symmetric additive category with translation.

A.6. Duality and nilpotence. In this subsection, we study symmetry of primitive parts under a (twisted) nilpotent operator. We formulate the problem in the language of duality on a category with translation introduced in Definition A.5.1. The main result of this subsection is Proposition A.6.8. This is applied in the main text to the logarithm of the monodromy operator associated to a normal crossing divisor to show that Grothendieck's six operations preserve $\mathrm{K}_{\text {orth }}$ (see the proof of Proposition 4.3.1).

Let $(\mathscr{A}, T)$ be an additive category with translation. In this subsection, we denote $T^{n} A$ by $A(n)$ instead of $A[n]$. Our first goal is to define a category of objects with nilpotent operators and record its relation with duality.

Construction A.6.1. Consider the additive category $\operatorname{Nil}(\mathscr{A}, T)$ of pairs $(A, N)$ of an object $A$ of $\mathscr{A}$ and a morphism $N: A(1) \rightarrow A$ which is nilpotent in the sense that there exists an integer $d \geq 0$ such that

$$
N^{d}:=N \circ N(1) \circ \cdots \circ N(d-1): A(d) \rightarrow A
$$

is the zero morphism. A morphism $(A, N) \rightarrow\left(A^{\prime}, N^{\prime}\right)$ is a morphism $f: A \rightarrow A^{\prime}$ of $\mathscr{A}$ satisfying $N^{\prime} f(1)=f N$.

There are two ways to identify $\operatorname{Nil}(\mathscr{A}, T)^{\mathrm{op}}$ and $\operatorname{Nil}\left(\mathscr{A}^{\mathrm{op}},\left(T^{\mathrm{op}}\right)^{-1}\right)$, which differ by a sign. We fix $\sigma= \pm 1$ and consider the isomorphism of categories

$$
E_{\mathscr{A}}=E_{(\mathscr{A}, T), \sigma}: \operatorname{Nil}(\mathscr{A}, T)^{\mathrm{op}} \rightarrow \operatorname{Nil}\left(\mathscr{A}^{\mathrm{op}},\left(T^{\mathrm{op}}\right)^{-1}\right)
$$

sending $(A, N: A(1) \rightarrow A)$ to $(A, \sigma N(-1): A \rightarrow A(-1))$. The composite

$$
\operatorname{Nil}(\mathscr{A}, T) \stackrel{E_{\mathscr{A}}^{\mathrm{op}}}{\longrightarrow} \operatorname{Nil}\left(\mathscr{A}^{\mathrm{op}},\left(T^{\mathrm{op}}\right)^{-1}\right)^{\mathrm{op}} \stackrel{E_{\mathscr{A}}^{\mathrm{op}}}{\longrightarrow} \operatorname{Nil}(\mathscr{A}, T)
$$

equals the identity. The duality we put on $\operatorname{Nil}(\mathscr{A}, T)$ will depend on the choice of $\sigma$. In the main text we take $\sigma=-1$.

Let $F:(\mathscr{A}, T) \rightarrow\left(\mathscr{A}^{\prime}, T^{\prime}\right)$ be a functor of additive categories with translation. Then $F$ induces an additive functor $\operatorname{Nil}_{F}: \operatorname{Nil}(\mathscr{A}, T) \rightarrow \operatorname{Nil}\left(\mathscr{A}^{\prime}, T^{\prime}\right)$ carrying $(A, N: T A \rightarrow A)$ to $\left(F A, T^{\prime} F A \simeq F T A \stackrel{F N}{\longrightarrow} F A\right)$ and $f:(A, N) \rightarrow\left(A^{\prime}, N^{\prime}\right)$ to $F f$. Let $\gamma: F \rightarrow F^{\prime}$ be a morphism of functors of categories with translation. Then $\gamma$ induces a natural transformation $\mathrm{Nil}_{\gamma}: \mathrm{Nil}_{F} \rightarrow \mathrm{Nil}_{F^{\prime}}$, which is a natural isomorphism if $\gamma$ is an isomorphism. 
The following diagrams commute:

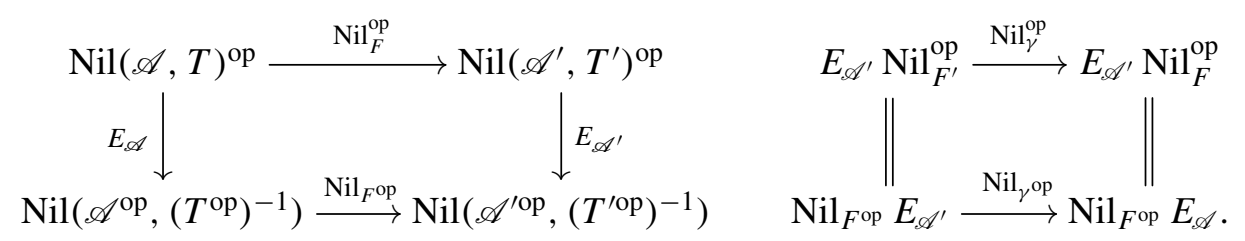

Construction A.6.2. Let $D:\left(\mathscr{A}^{\mathrm{op}},\left(T^{\mathrm{op}}\right)^{-1}\right) \rightarrow(\mathscr{A}, T)$ be an additive duality on the additive category with translation (see the comment following Definition A.5.1). Consider the functor $D_{\mathrm{Nil}(\mathscr{A}, T)}$, composite of

$$
\operatorname{Nil}(\mathscr{A}, T)^{\text {op }} \stackrel{E_{\mathscr{A}}}{\sim} \operatorname{Nil}\left(\mathscr{A}^{\mathrm{op}},\left(T^{\mathrm{op}}\right)^{-1}\right) \stackrel{\operatorname{Nil}_{D}}{\longrightarrow} \operatorname{Nil}(\mathscr{A}, T),
$$

and the natural transformation

$$
\begin{aligned}
\operatorname{id}_{\mathrm{Nil}(\mathscr{A}, T)} \stackrel{\mathrm{Nil}_{\mathrm{ev}}}{\longrightarrow} \operatorname{Nil}_{D} \mathrm{Nil}_{D^{\mathrm{op}}}=\mathrm{Nil}_{D} \mathrm{Nil}_{D^{\mathrm{op}}} E_{\mathscr{A} \text { op }} E_{\mathscr{A}}^{\mathrm{op}} \\
\quad=\operatorname{Nil}_{D} E_{\mathscr{A}} \mathrm{Nil}_{D}^{\mathrm{op}} E_{\mathscr{A}}^{\mathrm{op}}=D_{\mathrm{Nil}(\mathscr{A}, T)} D_{\mathrm{Nil}(\mathscr{A}, T)}^{\mathrm{op}} .
\end{aligned}
$$

These define an additive duality on the additive category $\operatorname{Nil}(\mathscr{A}, T)$, which is strong if $D$ is strong on $\mathscr{A}$.

In the rest of this section, let $(\mathscr{A}, T)$ be an abelian category with translation, namely an additive category with translation whose underlying category $\mathscr{A}$ is abelian. Our next goal is to review the decomposition into primitive parts. The following is a variant of [Deligne 1980, Proposition 1.6.1, 1.6.14], with essentially the same proof.

Lemma A.6.3. Let $(A, N)$ be an object of $\operatorname{Nil}(\mathscr{A}, T)$. Then there exists a unique finite increasing filtration $M$ of $A$ satisfying $N M_{j}(1) \subseteq M_{j-2}$ and such that $N^{k}$ induces an isomorphism $\mathrm{gr}_{k}^{M} A(k) \stackrel{\sim}{\longrightarrow} \mathrm{gr}_{-k}^{M} A$, for $k \geq 0$.

Proof. Let $d \geq 0$ be an integer such that $N^{d+1}=0$. We proceed by induction on $d$. We have $M_{d}=A$ and $M_{-d-1}=0$. For $d>0$, we have $M_{d-1}=\operatorname{Ker}\left(N^{d}\right)(-d)$ and $M_{-d}=\operatorname{Im}\left(N^{d}\right)$. We have $N^{d}=0$ on $\operatorname{Ker}\left(N^{d}\right)(-d) / \operatorname{Im}\left(N^{d}\right)$. Let $M^{\prime}$ be the corresponding filtration given by the induction hypothesis. Then, for $-d \leq i \leq d-1$, $M_{i}$ is the inverse image in $\operatorname{Ker}\left(N^{d}\right)(-d)$ of $M_{i}^{\prime} \subseteq \operatorname{Ker}\left(N^{d}\right)(-d) / \operatorname{Im}\left(N^{d}\right)$.

The following is an immediate consequence of the construction of the filtration $M$.

Lemma A.6.4. Let $f:(A, N) \rightarrow\left(A^{\prime}, N^{\prime}\right)$ be a morphism of $\operatorname{Nil}(\mathscr{A}, T)$. Then $f$ is compatible with the corresponding filtrations. More precisely, if $M$ and $M^{\prime}$ denote the corresponding filtrations, then $f\left(M_{j}\right) \subseteq M_{j}^{\prime}$.

For $i \leq 0$, let $P_{i}(A, N)=\operatorname{Ker}\left(N: \operatorname{gr}_{i}^{M} A(1) \rightarrow \operatorname{gr}_{i-2}^{M} A\right)(-1)$. The inclusion $\operatorname{Ker}(N)(-1) \subseteq A$ induces an isomorphism $\operatorname{gr}_{i}^{M}(\operatorname{Ker}(N)(-1)) \stackrel{\sim}{\longrightarrow} P_{i}(A, N)$. We 
thus obtain functors

$$
P_{i}=P_{i, \mathscr{A}}: \operatorname{Nil}(\mathscr{A}, T) \rightarrow \mathscr{A}
$$

For all $j$, we have

$$
\operatorname{gr}_{j}^{M} A \simeq \bigoplus_{\substack{k \geq|j| \\ k \equiv j(\bmod 2)}} P_{-k}(A, N)\left(-\frac{j+k}{2}\right) .
$$

We now proceed to define form transformations on the primitive part functors. Let $(A, N)$ be an object of $\operatorname{Nil}(\mathscr{A}, T)$. If $M=M(A, N)$ and $M^{*}=M\left(E_{\mathscr{A}}(A, N)\right)$, then we have the following short exact sequence in $\mathscr{A}$ :

$$
0 \rightarrow M_{-j-1} \rightarrow A \rightarrow M_{j}^{*} \rightarrow 0 .
$$

Thus $\operatorname{gr}_{-j}^{M} A$ can be identified with $\operatorname{gr}_{j}^{M^{*}} A$. Moreover, $N^{-i}: \mathrm{gr}_{-i}^{M} A(-i) \stackrel{\sim}{\rightarrow} \operatorname{gr}_{i}^{M} A$ induces an isomorphism in $\mathscr{A}$

$$
\begin{aligned}
\alpha_{\mathscr{A}}(A, N): P_{i, \mathscr{A} \text { op }}\left(E_{\mathscr{A}}(A, N)\right)(-i) \simeq \operatorname{gr}_{-i}^{M}(\operatorname{Coker}(N))(-i) & \\
& \stackrel{\sim}{\longrightarrow} \operatorname{gr}_{i}^{M}(\operatorname{Ker}(N)(-1)) \simeq P_{i, \mathscr{A}}(A, N) .
\end{aligned}
$$

This defines a natural isomorphism of functors $\alpha_{\mathscr{A}}: P_{i, \mathscr{A}}^{\mathrm{op}} \stackrel{\sim}{\longrightarrow}\left(T^{\mathrm{op}}\right)^{-i} P_{i, \mathscr{A} \text { op }} E_{\mathscr{A}}$. By definition, we have the following.

Lemma A.6.5. The isomorphism $T^{-i} P_{i, \mathscr{A} \text { op }}^{\mathrm{op}} E_{\mathscr{A}}^{\mathrm{op}} \stackrel{\alpha_{\mathscr{A}}^{\mathrm{op}}}{\sim} P_{i, \mathscr{A}} E_{\mathscr{A} \text { op }} E_{\mathscr{A}}^{\mathrm{op}}=P_{i, \mathscr{A}}$ is $\sigma^{i} \alpha_{\mathscr{A}}^{\mathrm{op}}$.

Let $(\mathscr{A}, T),\left(\mathscr{A}^{\prime}, T^{\prime}\right)$ be abelian categories with translation and let $F:(\mathscr{A}, T) \rightarrow$ $\left(\mathscr{A}^{\prime}, T^{\prime}\right)$ be a functor of categories with translation such that the underlying functor $\mathscr{A} \rightarrow \mathscr{A}^{\prime}$ is exact. Let $(A, N)$ be an object of $(\mathscr{A}, T)$ and let $M=M(A, N)$, $M^{\prime}=M\left(\operatorname{Nil}_{F}(A, N)\right)$. The exactness of $F$ allows us to identify $F\left(M_{j} A\right)$ as a subobject of $F A$, and under this identification we have $F\left(M_{j} A\right)=M_{j}^{\prime}(F A)$. We have an obvious natural isomorphism $\beta_{F}: P_{i, \mathscr{A}^{\prime}} \mathrm{Nil}_{F} \stackrel{\sim}{\longrightarrow} F P_{i, \mathscr{A}}$. The following functoriality of $\beta$ is obvious.

Lemma A.6.6. Let $F, F^{\prime}:(\mathscr{A}, T) \rightarrow\left(\mathscr{A}^{\prime}, T^{\prime}\right)$ be functors of categories with translation such that the underlying functors are exact, and let $\gamma: F \rightarrow F^{\prime}$ be a morphism of functors of categories with translation. Then the following diagram commutes:

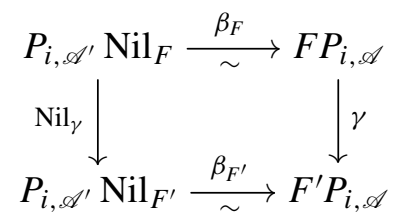

By construction, the isomorphisms $\alpha$ and $\beta$ have the following compatibility. 
Lemma A.6.7. The following diagram commutes:

$$
\begin{aligned}
&\left(T^{\prime \mathrm{op}}\right)^{-i} F^{\mathrm{op}} P_{i, \mathscr{A}} \mathrm{op} E_{\mathscr{A}} \longleftarrow F^{\mathrm{op}}\left(T^{\mathrm{op}}\right)^{-i} P_{i, \mathscr{A}} \text { op } E_{\mathscr{A}} \stackrel{\alpha_{\mathscr{A}}}{\sim} F^{\mathrm{op}} P_{i, \mathscr{A}}^{\mathrm{op}} \simeq \beta_{F}^{\mathrm{op}} \\
& \beta_{F^{\mathrm{op}}} \uparrow \simeq \simeq \\
&\left(T^{\prime \mathrm{op}}\right)^{-i} P_{i, \mathscr{A}^{\prime} \mathrm{op}} \operatorname{Nil}_{F^{\mathrm{op}}} E_{\mathscr{A}} \simeq\left(T^{\prime \mathrm{op}}\right)^{-i} P_{i, \mathscr{A}^{\prime} \mathrm{op}} E_{\mathscr{A}^{\prime}} \mathrm{Nil}_{F}^{\mathrm{op}} \stackrel{\alpha_{\mathscr{A}^{\prime}}}{\sim} P_{\mathscr{A}^{\prime}}^{\mathrm{op}} \mathrm{Nil}_{F}^{\mathrm{op}}
\end{aligned}
$$

The following is the main result of this subsection.

Proposition A.6.8. Let $(\mathscr{A}, T)$ be an abelian category with translation and let $D:\left(\mathscr{A}^{\mathrm{op}},\left(T^{\mathrm{op}}\right)^{-1}\right) \rightarrow(\mathscr{A}, T)$ be a duality such that the underlying functor $\mathscr{A}^{\mathrm{op}} \rightarrow \mathscr{A}$ is exact. Then, for $i \leq 0$, the composite isomorphism

$$
P_{i, \mathscr{A}} D_{\mathrm{Nil}(\mathscr{A}, T)}=P_{i, \mathscr{A}} \mathrm{Nil}_{D} E_{\mathscr{A}} \stackrel{\beta_{D}}{\sim} D P_{i, \mathscr{A}} \mathrm{op} E_{\mathscr{A}} \stackrel{\alpha_{\mathscr{A}}^{-1}}{\sim}\left(D\left(T^{\mathrm{op}}\right)^{i}\right) P_{i, \mathscr{A}}^{\mathrm{op}}
$$

is $\sigma^{i}$-symmetric.

Note that $T^{-i} D \simeq D\left(T^{\mathrm{op}}\right)^{i}: \mathscr{A}^{\mathrm{op}} \rightarrow \mathscr{A}$ endowed with the natural transformation $\operatorname{id}_{\mathscr{A}} \stackrel{\mathrm{ev}}{\longrightarrow} D D^{\mathrm{op}} \simeq\left(D\left(T^{\mathrm{op}}\right)^{i}\right)\left(T^{-i} D\right)^{\mathrm{op}}$ is a duality on $\mathscr{A}$. By the proposition, $P_{i, \mathscr{A}}$ carries $\sigma^{\prime}$-self-dual objects of $\operatorname{Nil}(\mathscr{A}, T)$ to $\sigma^{i} \sigma^{\prime}$-self-dual objects of $\mathscr{A}$.

Proof. In the diagram

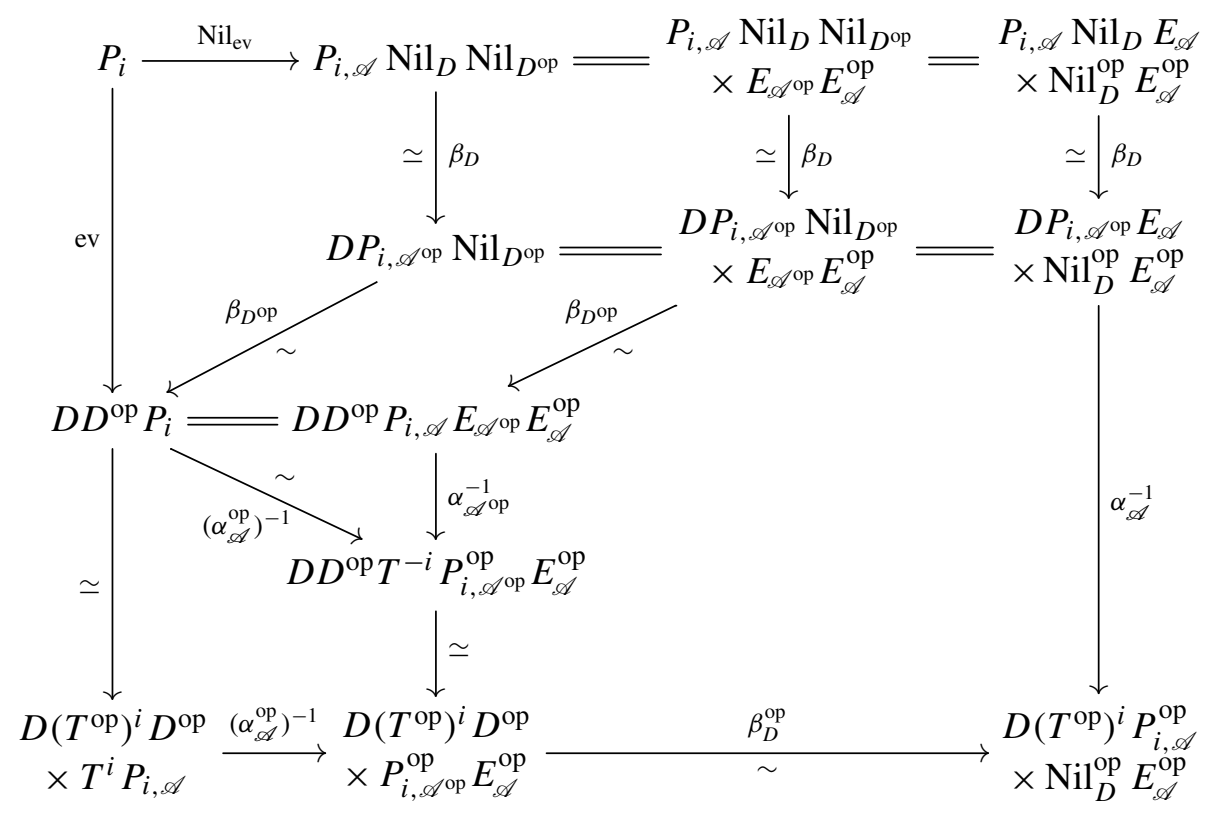

the triangle $\sigma^{i}$-commutes by Lemma A.6.5, the upper-left inner cell commutes by Lemma A.6.6, the lower-right inner cell commutes by Lemma A.6.7, and the other inner cells trivially commute. 


\section{Acknowledgements}

We thank Luc Illusie for encouragement and enlightening conversations. We thank Ofer Gabber for many helpful suggestions, and we are grateful to him and Gérard Laumon for pointing out a mistake in an earlier draft of this paper. We are indebted to Takeshi Saito for fruitful suggestions on general base fields, to Pierre Deligne for generously sharing his knowledge on $\lambda$-rings, to Jiangxue Fang for bringing our attention to Kashiwara's conjecture, and to Matthew Young for suggesting a connection to Grothendieck-Witt groups. Sun thanks Torsten Ekedahl, Bernd Ulrich, and many others for helpful discussions on the MathOverflow website. Zheng thanks Michel Brion, Zongbin Chen, Lei Fu, Michel Gros, Binyong Sun, Yichao Tian, Claire Voisin, Liang Xiao, and Zhiwei Yun for useful discussions. Part of this work was done during various stays of the authors at Université Paris-Sud, Institut des Hautes Études Scientifiques, Korea Institute for Advanced Studies, Shanghai Jiao Tong University, and Hong Kong University of Science and Technology. We thank these institutions for hospitality and support. We thank the referees for

\section{References}

[Atiyah 1956] M. F. Atiyah, "On the Krull-Schmidt theorem with application to sheaves", Bull. Soc. Math. France 84 (1956), 307-317. MR 19,172b Zbl 0072.18101

[Atiyah and Tall 1969] M. F. Atiyah and D. O. Tall, "Group representations, $\lambda$-rings and the $J$ homomorphism", Topology 8 (1969), 253-297. MR 39 \#5702 Zbl 0159.53301

[Beilinson et al. 1982] A. A. Beilinson, J. Bernstein, and P. Deligne, "Faisceaux pervers", pp. 5171 in Analyse et topologie sur les espaces singuliers, I (Luminy, 1981), Astérisque 100, Société Mathématique de France, Paris, 1982. MR 86g:32015 Zbl 0536.14011

[Bröcker and tom Dieck 1995] T. Bröcker and T. tom Dieck, Representations of compact Lie groups, Graduate Texts in Mathematics 98, Springer, New York, NY, 1995. MR 97i:22005 Zbl 0874.22001

[Calmès and Hornbostel 2009] B. Calmès and J. Hornbostel, "Tensor-triangulated categories and dualities”, Theory Appl. Categ. 22:6 (2009), 136-200. MR 2010k:18008 Zbl 1178.18005

[Chevalley 1955] C. Chevalley, Théorie des groupes de Lie, III: Théorèmes généraux sur les algèbres de Lie, Actualités Scientifiques et Industrielles 1226, Hermann, Paris, 1955. MR 16,901a Zbl 0186.33104

[Conrad et al. 2012] B. Conrad, M. Lieblich, and M. Olsson, "Nagata compactification for algebraic spaces”, J. Inst. Math. Jussieu 11:4 (2012), 747-814. MR 2979821 Zbl 1255.14003

[Deligne 1968] P. Deligne, “Théorème de Lefschetz et critères de dégénérescence de suites spectrales", Inst. Hautes Études Sci. Publ. Math. 35 (1968), 107-126. MR 39 \#5582 Zbl 0159.22501

[Deligne 1980] P. Deligne, "La conjecture de Weil, II", Inst. Hautes Études Sci. Publ. Math. 52 (1980), 137-252. MR 83c:14017 Zbl 0456.14014

[Deligne 1994] P. Deligne, "Décompositions dans la catégorie dérivée", pp. 115-128 in Motives (Seattle, WA, 1991), edited by U. Jannsen et al., Proceedings of Symposia in Pure Mathematics 55, American Mathematical Society, Providence, RI, 1994. MR 95h:18013 Zbl 0809.18008

[Deligne 2012] P. Deligne, "Finitude de l'extension de $\mathbb{Q}$ engendrée par des traces de Frobenius, en caractéristique finie”, Mosc. Math. J. 12:3 (2012), 497-514. MR 3024820 Zbl 1260.14022 
[Drinfeld 2001] V. Drinfeld, "On a conjecture of Kashiwara", Math. Res. Lett. 8:5-6 (2001), 713-728. MR 2003c:14022 Zbl 1079.14509

[Drinfeld 2012] V. Drinfeld, "On a conjecture of Deligne”, Mosc. Math. J. 12:3 (2012), 515-542. MR 3024821 Zbl 1271.14028

[Fujiwara 2002] K. Fujiwara, "Independence of $l$ for intersection cohomology (after Gabber)", pp. 145-151 in Algebraic geometry 2000, Azumino (Nagano, 2000), edited by S. Usui et al., Advanced Studies in Pure Mathematics 36, Mathematical Society of Japan, Tokyo, 2002. MR 2004c:14038 Zbl 1057.14029

[Gaitsgory 2007] D. Gaitsgory, “On de Jong's conjecture”, Israel J. Math. 157 (2007), 155-191. MR 2008j:14021 Zbl 1123.11020

[Grothendieck 1958] A. Grothendieck, "La théorie des classes de Chern", Bull. Soc. Math. France 86 (1958), 137-154. MR 22 \#6818 Zbl 0091.33201

[Grothendieck 1961] A. Grothendieck, "Éléments de géométrie algébrique, II: Étude globale élémentaire de quelques classes de morphismes", Inst. Hautes Études Sci. Publ. Math. 8 (1961), 5-222. MR 36 \#177b Zbl 0118.36206

[Grothendieck 1977] A. Grothendieck, "Formule de Lefschetz", exposé III, rédigé par L. Illusie, pp. 73-137 in Séminaire de Géometrie Algébrique du Bois-Marie (SGA 5): cohomologie l-adique et fonctions L (Bures-sur-Yvette, 1965-1966), edited by L. Illusie, Lecture Notes in Mathematics 589, Springer, Berlin, 1977. MR 58 \#10907 Zbl 0355.14004

[Huber 1997] A. Huber, "Mixed perverse sheaves for schemes over number fields", Compos. Math. 108:1 (1997), 107-121. MR 98k:14024 Zbl 0882.14006

[Illusie 1994] L. Illusie, "Autour du théorème de monodromie locale", pp. 9-57 in Périodes p-adiques (Bures-sur-Yvette, 1988), edited by J.-M. Fontaine, Astérisque 223, Société Mathématique de France, Paris, 1994. MR 95k:14032 Zbl 0837.14013

[Illusie and Zheng 2013] L. Illusie and W. Zheng, "Odds and ends on finite group actions and traces", Int. Math. Res. Not. 2013:1 (2013), 1-62. MR 3041694 Zbl 06132684

[de Jong 1996] A. J. de Jong, "Smoothness, semi-stability and alterations", Inst. Hautes Études Sci. Publ. Math. 83 (1996), 51-93. MR 98e:14011 Zbl 0916.14005

[de Jong 1997] A. J. de Jong, "Families of curves and alterations", Ann. Inst. Fourier (Grenoble) 47:2 (1997), 599-621. MR 98f:14019 Zbl 0868.14012

[Kashiwara and Schapira 2006] M. Kashiwara and P. Schapira, Categories and sheaves, Grundlehren der Mathematischen Wissenschaften 332, Springer, Berlin, 2006. MR 2006k:18001 Zbl 1118.18001

[Katz 2005] N. M. Katz, Moments, monodromy, and perversity: a Diophantine perspective, Annals of Mathematics Studies 159, Princeton University Press, 2005. MR 2006j:14020 Zbl 1079.14025

[Keel and Mori 1997] S. Keel and S. Mori, "Quotients by groupoids", Ann. of Math. (2) 145:1 (1997), 193-213. MR 97m:14014 Zbl 0881.14018

[Kleiman 1994] S. L. Kleiman, "The standard conjectures", pp. 3-20 in Motives (Seattle, WA, 1991), edited by U. Jannsen et al., Proc. Sympos. Pure Math. 55, American Mathematical Society, Providence, RI, 1994. MR 95k:14010 Zbl 0820.14006

[Lafforgue 2002] L. Lafforgue, "Chtoucas de Drinfeld et correspondance de Langlands", Invent. Math. 147:1 (2002), 1-241. MR 2002m:11039 Zbl 1038.11075

[Laumon 1981] G. Laumon, "Comparaison de caractéristiques d'Euler-Poincaré en cohomologie l-adique”, C. R. Acad. Sci. Paris Sér. I Math. 292:3 (1981), 209-212. MR 82e:14030 Zbl 0468.14005

[Laumon 1987] G. Laumon, "Transformation de Fourier, constantes d'équations fonctionnelles et conjecture de Weil", Inst. Hautes Études Sci. Publ. Math. 65 (1987), 131-210. MR 88g:14019 Zbl 0641.14009 
[Laumon and Moret-Bailly 2000] G. Laumon and L. Moret-Bailly, Champs algébriques, Ergebnisse der Mathematik und ihrer Grenzgebiete (3) 39, Springer, Berlin, 2000. MR 2001f:14006 Zbl 0945.14005

[Lurie 2014] J. Lurie, "Higher algebra", preprint, 2014, available at http://www.math.harvard.edu/ $\sim$ lurie/papers/higheralgebra.pdf.

[Mac Lane 1998] S. Mac Lane, Categories for the working mathematician, 2nd ed., Graduate Texts in Mathematics 5, Springer, New York, NY, 1998. MR 2001j:18001 Zbl 0906.18001

[Morel 2012] S. Morel, “Complexes mixtes sur un schéma de type fini sur $\mathbb{Q}$ ”, preprint, 2012, available at http://web.math.princeton.edu/ smorel/sur_Q.pdf.

[Pacheco and Stevenson 2000] A. Pacheco and K. F. Stevenson, "Finite quotients of the algebraic fundamental group of projective curves in positive characteristic", Pacific J. Math. 192:1 (2000), 143-158. MR 2000k:14023 Zbl 0951.14016

[Partsch 2013] H. Partsch, "Deformations of elliptic fiber bundles in positive characteristic", Nagoya Math. J. 211 (2013), 79-108. MR 3079280 Zbl 1274.14044

[Quebbemann et al. 1979] H.-G. Quebbemann, W. Scharlau, and M. Schulte, "Quadratic and Hermitian forms in additive and abelian categories”, J. Algebra 59:2 (1979), 264-289. MR 82d:18015 Zbl 0412.18016

[Quillen 1971] D. Quillen, “The Adams conjecture”, Topology 10 (1971), 67-80. MR 43 \#5525 Zbl 0219.55013

[Raynaud and Gruson 1971] M. Raynaud and L. Gruson, "Critères de platitude et de projectivité: techniques de «platification» d'un module", Invent. Math. 13:1 (1971), 1-89. MR 46 \#7219 Zbl 0227.14010

[Riou 2014] J. Riou, “Dualité”, exposé XVII, pp. 351-453 in Travaux de Gabber sur l'uniformisation locale et la cohomologie étale des schémas quasi-excellents (Palaiseau, 2006-2008), edited by L. Illusie et al., Astérisque 363-364, Société Mathématique de France, Paris, 2014. MR 3329787 Zbl 1320.14032

[Saito 1990] M. Saito, "Mixed Hodge modules", Publ. Res. Inst. Math. Sci. 26:2 (1990), 221-333. MR 91m:14014 Zbl 0727.14004

[Schlichting 2010] M. Schlichting, "Hermitian $K$-theory of exact categories", J. K-Theory 5:1 (2010), 105-165. MR 2011b:19007 Zbl 05690542

[Serre 1965] J.-P. Serre, "Zeta and L functions", pp. 82-92 in Arithmetical algebraic geometry (Purdue, IN, 1963), edited by O. F. G. Schilling, Harper \& Row, New York, NY, 1965. MR 33 \#2606 Zbl 0171.19602

[Serre 1998] J.-P. Serre, Représentations linéaires des groupes finis, 5th ed., Hermann, Paris, 1998. 2nd ed., 1971, translated as Linear representations of finite groups, Graduate Texts in Mathematics 42, Springer, New York, 1977. MR 80f:20001 Zbl 0926.20003

[Suh 2012] J. Suh, "Symmetry and parity in Frobenius action on cohomology", Compos. Math. 148:1 (2012), 295-303. MR 2881317 Zbl 1258.14023

[Sun 2012a] S. Sun, "Decomposition theorem for perverse sheaves on Artin stacks over finite fields", Duke Math. J. 161:12 (2012), 2297-2310. MR 2972459 Zbl 1312.14057

[Sun 2012b] S. Sun, "L-series of Artin stacks over finite fields", Algebra Number Theory 6:1 (2012), 47-122. MR 2950161 Zbl 06064705

[Verdier 1996] J.-L. Verdier, Des catégories dérivées des catégories abéliennes, edited by G. Maltsiniotis, Astérisque 239, Société Mathématique de France, Paris, 1996. MR 98c:18007 Zbl 0882.18010 
[Vidal 2004] I. Vidal, “Théorie de Brauer et conducteur de Swan”, J. Algebraic Geom. 13:2 (2004), 349-391. MR 2005m:14030 Zbl 1070.14020

[Zheng 2005] W. Zheng, "Théorème de Gabber d'indépendance de l", Master's thesis, Université Paris-Sud, Orsay, 2005, available at http://159.226.47.28/ zheng/memoire.pdf.

[Zheng 2008] W. Zheng, "Sur la cohomologie des faisceaux $l$-adiques entiers sur les corps locaux", Bull. Soc. Math. France 136:3 (2008), 465-503. MR 2009d:14015 Zbl 1216.14016

[Zheng 2009] W. Zheng, "Sur l'indépendance de $l$ en cohomologie $l$-adique sur les corps locaux", Ann. Sci. Éc. Norm. Supér. (4) 42:2 (2009), 291-334. MR 2010i:14032 Zbl 1203.14023

[Zheng 2015a] W. Zheng, "Companions on Artin stacks”, preprint, 2015. arXiv 1512.08929

[Zheng 2015b] W. Zheng, "Six operations and Lefschetz-Verdier formula for Deligne-Mumford stacks”, Sci. China Math. 58:3 (2015), 565-632. MR 3319927 Zbl 06430227

Communicated by Brian Conrad

Received 2014-08-26 Revised 2015-10-02 Accepted 2015-12-31

shsun@math.tsinghua.edu.cn Yau Mathematical Sciences Center, Tsinghua University, Jinchunyuan West Building, Beijing, 100084, China

wzheng@math.ac.cn

Morningside Center of Mathematics, Academy of Mathematics and Systems Science, Chinese Academy of Sciences, Zhongguancun Donglu 55, Beijing, 100190, China 


\section{Algebra \& Number Theory}

msp.org/ant

\section{EDITORS}

MANAGING EDITOR

Bjorn Poonen

Massachusetts Institute of Technology

Cambridge, USA

\author{
EDITORIAL BOARD CHAIR \\ David Eisenbud \\ University of California \\ Berkeley, USA
}

BOARD OF EDITORS

Georgia Benkart

Dave Benson

Richard E. Borcherds

John H. Coates

J-L. Colliot-Thélène

Brian D. Conrad

Hélène Esnault

Hubert Flenner

Sergey Fomin

Edward Frenkel

Andrew Granville

Joseph Gubeladze

Roger Heath-Brown

Craig Huneke

Kiran S. Kedlaya

János Kollár

Yuri Manin

Philippe Michel
University of Wisconsin, Madison, USA

University of Aberdeen, Scotland

University of California, Berkeley, USA

University of Cambridge, UK

CNRS, Université Paris-Sud, France

Stanford University, USA

Freie Universität Berlin, Germany

Ruhr-Universität, Germany

University of Michigan, USA

University of California, Berkeley, USA

Université de Montréal, Canada

San Francisco State University, USA

Oxford University, UK

University of Virginia, USA

Univ. of California, San Diego, USA

Princeton University, USA

Northwestern University, USA

École Polytechnique Fédérale de Lausanne
Susan Montgomery

Shigefumi Mori

Raman Parimala

Jonathan Pila

Anand Pillay

Victor Reiner

Peter Sarnak

Joseph H. Silverman

Michael Singer

Vasudevan Srinivas

J. Toby Stafford

Ravi Vakil

Michel van den Bergh

Marie-France Vignéras

Kei-Ichi Watanabe

Efim Zelmanov

Shou-Wu Zhang
University of Southern California, USA

RIMS, Kyoto University, Japan

Emory University, USA

University of Oxford, UK

University of Notre Dame, USA

University of Minnesota, USA

Princeton University, USA

Brown University, USA

North Carolina State University, USA

Tata Inst. of Fund. Research, India

University of Michigan, USA

Stanford University, USA

Hasselt University, Belgium

Université Paris VII, France

Nihon University, Japan

University of California, San Diego, USA

Princeton University, USA

\section{PRODUCTION}

production@msp.org

Silvio Levy, Scientific Editor

See inside back cover or msp.org/ant for submission instructions.

The subscription price for 2016 is US $\$ 290 /$ year for the electronic version, and $\$ 485 /$ year ( $+\$ 55$, if shipping outside the US) for print and electronic. Subscriptions, requests for back issues and changes of subscribers address should be sent to MSP.

Algebra \& Number Theory (ISSN 1944-7833 electronic, 1937-0652 printed) at Mathematical Sciences Publishers, 798 Evans Hall \#3840, c/o University of California, Berkeley, CA 94720-3840 is published continuously online. Periodical rate postage paid at Berkeley, CA 94704, and additional mailing offices.

ANT peer review and production are managed by EditFLOW ${ }^{\circledR}$ from MSP.

\section{PUBLISHED BY}

- mathematical sciences publishers

nonprofit scientific publishing

http://msp.org/

(C) 2016 Mathematical Sciences Publishers 


\section{Algebra \& Number Theory}

Volume $10 \quad$ No. $2 \quad 2016$

Kummer theory for Drinfeld modules

RICHARD PINK

Parity and symmetry in intersection and ordinary cohomology

SHENGHAO SUN and WeIZHE ZHENG

DANIEL KRIZ

Squarefree polynomials and Möbius values in short intervals and arithmetic progressions JONATHAN P. KEATING and ZEEV RUDNICK

Equidistribution of values of linear forms on a cubic hypersurface SAM CHOW 